\title{
Ni-catalyzed Iterative Alkyl Transfer from Nitrogen Enabled by the In Situ Methylation of Tertiary Amines
}

Chideraa Iheanyi Nwachukwu, ${ }^{+}$Timothy Patrick McFadden ${ }^{+}$and Andrew George Roberts*,+ +Department of Chemistry, University of Utah, 315 South 1400 East, Salt Lake City, Utah 84112 , United States

\section{Supporting Information}

\section{Table of Contents}

I. Optimization of $\mathrm{Ni}$-catalyzed homodimerization reactions

II. General procedure for preparation of di, tri and tetra- benzyl ammonium iodides $(8,9,10,14)$

III. NMR trace of crude reaction mixture for iterative alkyl group transfer

IV. Ni-catalyzed evaluation of unsymmetric tertiary amine substrates

V. ${ }^{1} \mathrm{H}$ and ${ }^{13} \mathrm{C}$ NMR spectra

VI. ${ }^{1} \mathrm{H}$ NMR and GC/MS trace for entry A in Fig. S5 


\section{Optimization of $\mathrm{Ni}$-catalyzed homodimerization reactions}

Initial screening experiments were executed with $\mathrm{NiBr}_{2}$ glyme as the pre-catalyst in combination with 5 equiv. of $\mathrm{Mn}$ at $120^{\circ} \mathrm{C}$ (oil bath). Table S1 shows screening experiments with 16 different ligands.

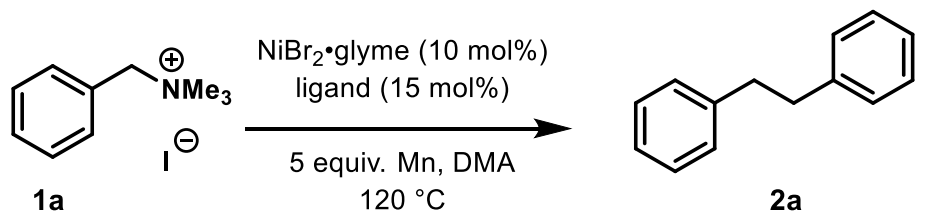

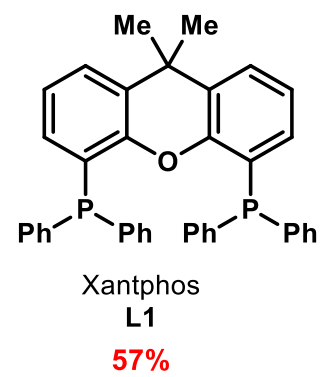

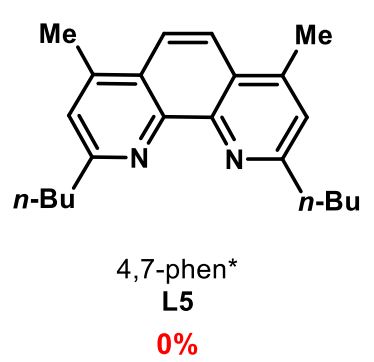<smiles>c1ccc(-c2ccccn2)nc1</smiles><smiles>c1ccc(P(CCCP(c2ccccc2)c2ccccc2)c2ccccc2)cc1</smiles><smiles>Cc1ccnc(-c2cc(C)ccn2)c1</smiles>

$$
\begin{gathered}
\text { 4,4'-bipy } \\
\text { L2 } \\
45 \%
\end{gathered}
$$<smiles>CC(C)(C)c1ccnc(-c2cc(C(C)(C)C)ccn2)c1</smiles>

4,4'-bipy

L3

$50 \%$<smiles>Cc1ccnc2c1ccc1c(C)ccnc12</smiles>

4,7-phen

L6

$5 \%$<smiles>c1ccc(-c2ccnc3c2ccc2c(-c4ccccc4)ccnc23)cc1</smiles>

bathophen

L7

$17 \%$<smiles>CC(C)(C)c1ccnc(-c2cc(C(C)(C)C)cc(-c3cc(C(C)(C)C)ccn3)n2)c1</smiles><smiles>Cl[PH](c1ccccc1)(c1ccccc1)c1ccccc1</smiles>

$\mathrm{PPh}_{2} \mathrm{Cy}$

L12

$20 \%$

$$
\begin{gathered}
\text { 2,2'-bipy } \\
\text { L9 } \\
24 \%
\end{gathered}
$$

\section{DPPP}

L13

$48 \%$<smiles>COc1ccnc(-c2cc(OC)ccn2)c1</smiles>

$$
\begin{array}{cc}
\text { 4,4'-bipy } & \text { DPPB } \\
\text { L10 } & \text { L14 } \\
20 \% & 52 \%
\end{array}
$$<smiles>Cc1cccc(-c2cccc(C)n2)n1</smiles>

$$
\begin{gathered}
4,4^{\prime}, 4^{\prime \prime} \text {-terpyr } \\
\text { L8 } \\
13 \%
\end{gathered}
$$<smiles>[SiH2][PH](C1CCCCC1)=P(Cl)(Cl)C1CCCCC1</smiles>

$\mathrm{PCy}_{3}$

L15

$18 \%$

Table S1. Ligand screening

Reaction conditions: 1a (1.34 mmol), Nil 2 (10mol\%), L1 (15mol\%), Mn (5 equiv., $4.02 \mathrm{mmol})$, DMA $(0.40 \mathrm{M})$ at $120^{\circ} \mathrm{C}$ for $24 \mathrm{~h}$. Yields of isolated $(2 \mathrm{a})$ provided as an average of at least two independent runs. 
II. General procedure for preparation of di, tri and tetra- benzyl ammonium iodides (8, $\underline{9,10,14)}$

Amine (1 equiv.) and alkyl iodide (1.5 equiv.) were dissolved in acetonitrile ( $1 \mathrm{M})$ in a round bottom flask. The entire mixture was refluxed $\left(90^{\circ} \mathrm{C}\right.$ in an oil bath) for $12 \mathrm{~h}$. The resultant precipitate was isolated by filtration and washed with ether $(10 \mathrm{~mL} \times 4)$ to afford the corresponding iodide salt which was used without further purification.

Entry Amine

Table S2. ${ }^{\mathrm{b}}$ Reaction performed in THF 


\section{NMR trace of crude reaction mixture for iterative alkyl group transfer}

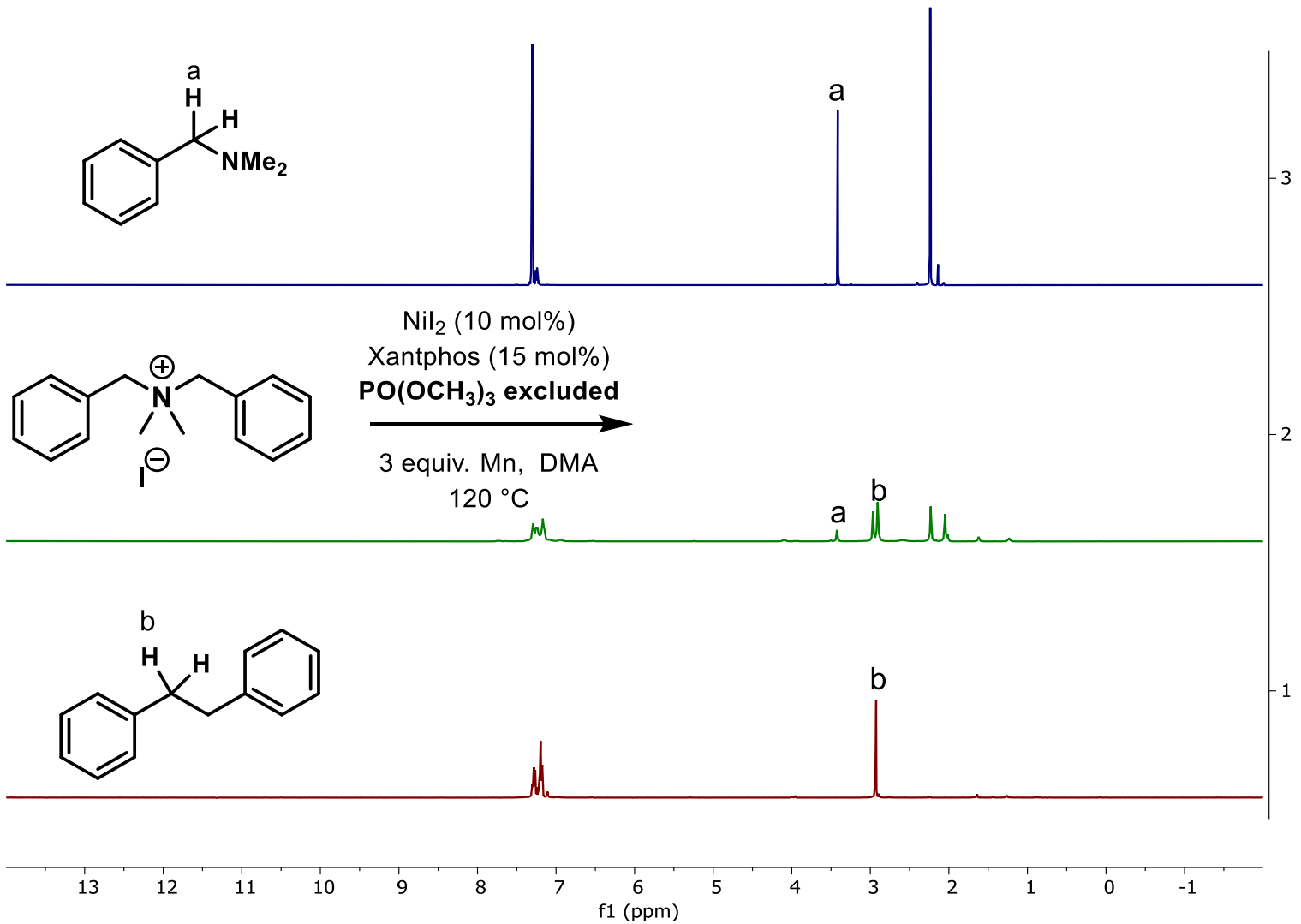

Fig S1. Crude NMR of iterative benzyl transfer without $\mathrm{PO}\left(\mathrm{OCH}_{3}\right)_{3}$; indicating the presence of dimethylbenzyl amine
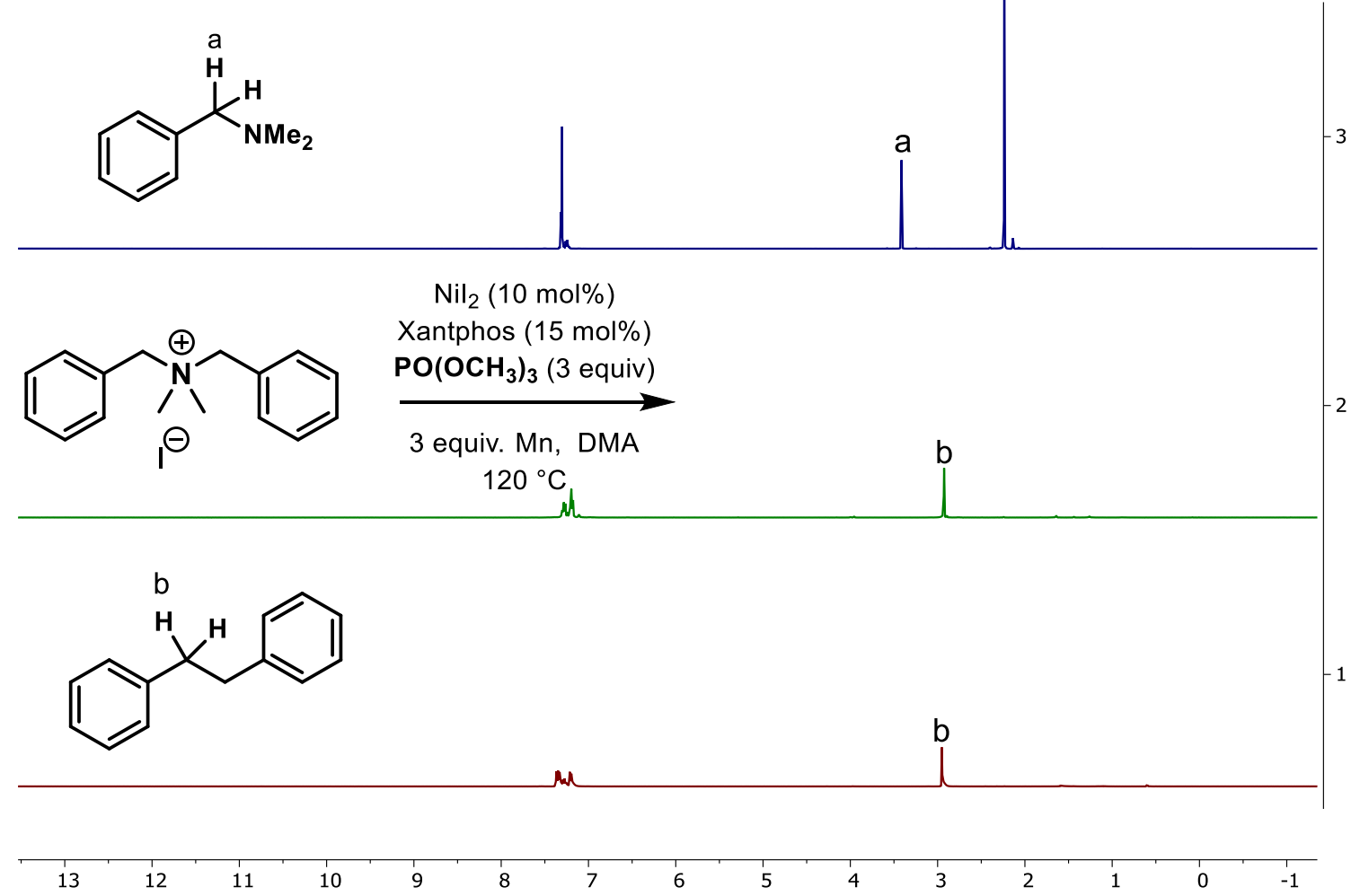

Fig S2. Crude NMR of iterative benzyl trânsfer (ppr $^{2}$ with $\mathrm{PO}\left(\mathrm{OCH}_{3}\right)_{3}$; indicating the absence of dimethylbenzyl amine 


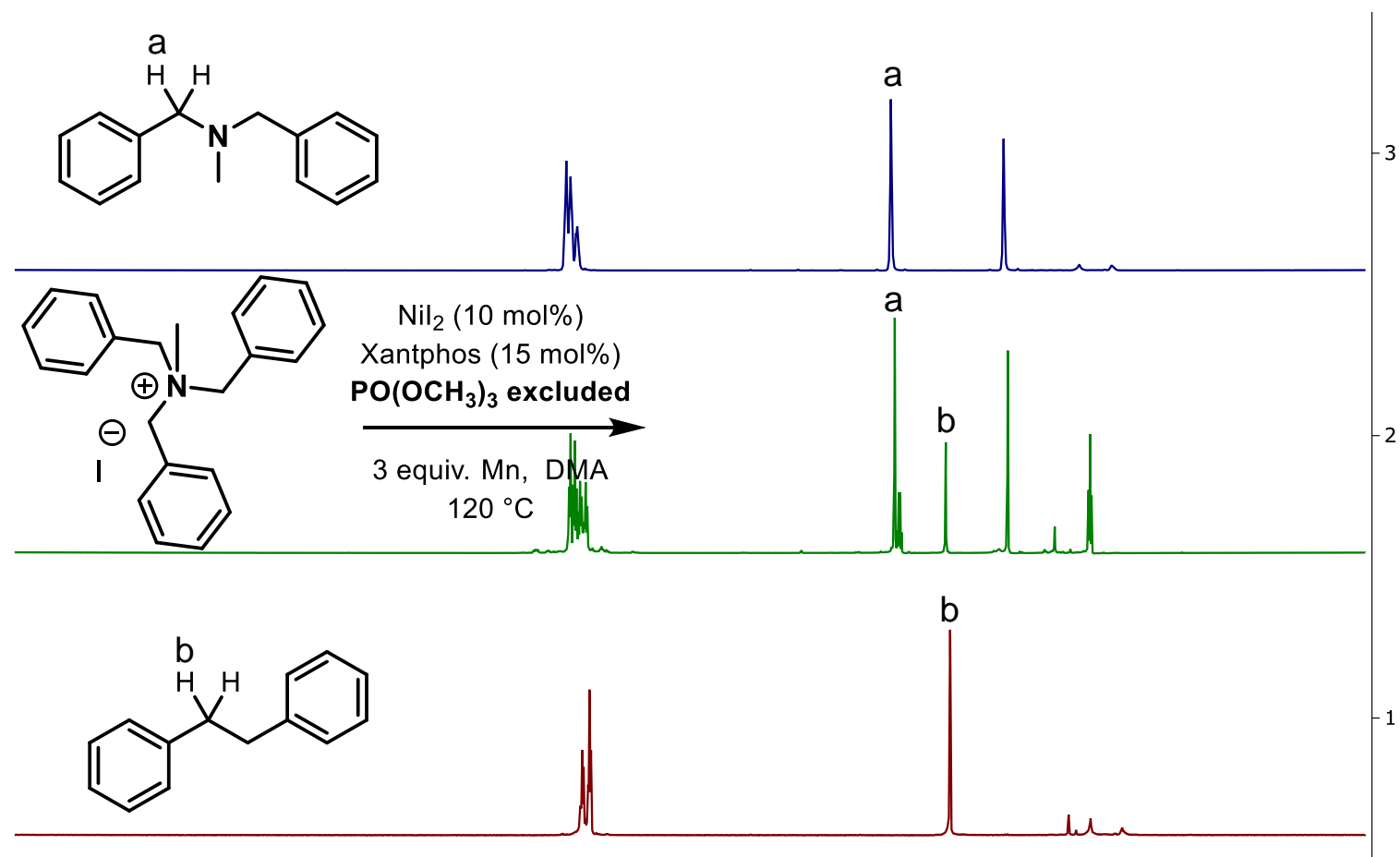$$
4
$$

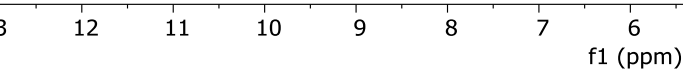

Fig S3. Crude NMR of iterative benzyl transfer without $\mathrm{PO}\left(\mathrm{OCH}_{3}\right)_{3}$; indicating the presence of $\mathrm{N}, \mathrm{N}$-dibenzyl-methylamine<smiles>CC(c1ccccc1)N(C)Cc1ccccc1</smiles>

$\mathrm{Nil}_{2}$ (10 mol\%)

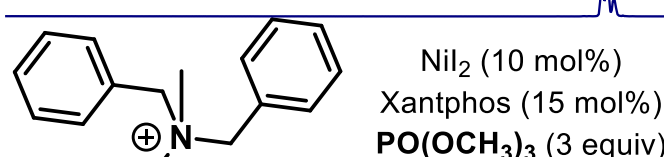

$\Theta$

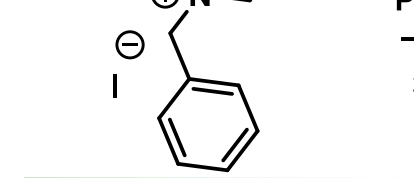
$\mathrm{PO}\left(\mathrm{OCH}_{3}\right)_{3}$ (3 equiv)

equiv. Mn, DMA $120^{\circ} \mathrm{C}$
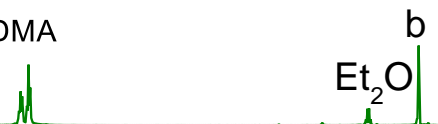

$\mathrm{Et}_{2} \mathrm{O}$<smiles>[2H]C(Cc1ccccc1)c1ccccc1</smiles>

b
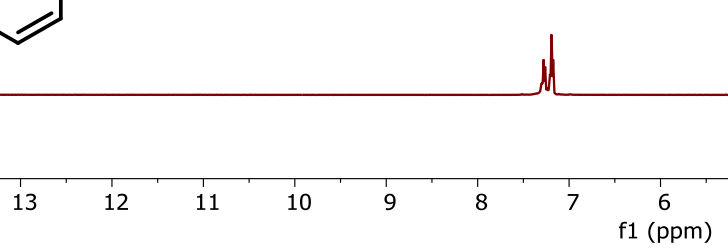

Fig S4. Crude NMR of iterative benzyl transfer with $\mathrm{PO}\left(\mathrm{OCH}_{3}\right)_{3}$; indicating the absence of $N, N$-dibenzyl-methylamine 


\section{Ni-catalyzed evaluation of unsymmetric tertiary amine substrates}

A $25 \mathrm{~mL}$ oven dried round bottom flask equipped with a magnetic stir bar was evacuated and charged with catalyst $\mathrm{Nil}_{2}(14 \mathrm{mg}, 0.044 \mathrm{mmol})$ and Xantphos $(38 \mathrm{mg}, 0.067 \mathrm{mmol})$ under positive nitrogen flow. Degassed DMA was added via a syringe and the mixture was stirred for $5 \mathrm{~min}$ at $120^{\circ} \mathrm{C}$ after which the solution became homogeneous and the color changed from grey to red. Mn (73 mg, $1.32 \mathrm{mmol}$ ), either benzyl amine, ammonium salt or benzyl bromide $(0.44 \mathrm{mmol})$, trimethylphosphate $(0.21 \mathrm{~mL}, 1.76 \mathrm{mmol})$ were added to the mixture. The flask was equipped with a condenser and the entire mixture stirred under nitrogen at either 25 or $120{ }^{\circ} \mathrm{C}$ (oil bath) for $24 \mathrm{~h}$. The mixture was allowed to cool to room temperature and subsequently extracted with diethyl ether $(10 \mathrm{~mL} \times 3)$. The combined organic extract was washed with water $(10 \mathrm{~mL} \times 2)$, followed by brine $(5 \mathrm{~mL})$. The organic layer was then dried over $\mathrm{Na}_{2} \mathrm{SO}_{4}$, filtered and solvent removed under reduced pressure. The crude mixture was passed through a short silica plug and analyzed by GC/MS. Ratios of the products are obtained by integrating the individual peaks corresponding to $\mathbf{1 6}, \mathbf{2 a}$ and $\mathbf{2 b}$. The $\mathrm{GC}$ data obtained is presented as a bar graph for better visual representation (Fig S5).

An example calculation of how the GC/MS data was normalized using entry A:

For 16: $(A \cup C)$ /sum (AUC: $16+2 a+2 b)=0.44$

For 2a: $(A \cup C) /$ sum $(A \cup C: 16+2 a+2 b)=0.10$

For 26: $(A \cup C) /$ sum $(A \cup C: 16+2 a+2 b)=0.46$

The data obtained from the GC/MS was also replicated by analysing the crude mixture via NMR. Due to chemical shift overlap in the NMR spectrum, we elected to analyzed all data using GC/MS. NMR spectrum and GC/MS chromatogram for entry A (representative) is shown on page $S 7$. 
a) mechanistic probe

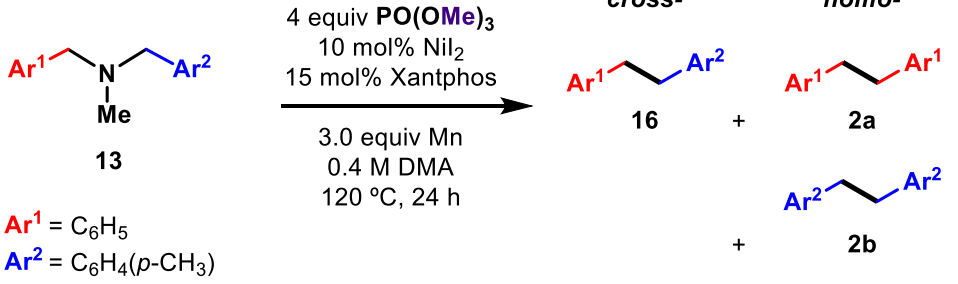

$=\mathrm{C}_{6} \mathrm{H}_{4}\left(p-\mathrm{CH}_{3}\right)$

b) evaluated substrates:

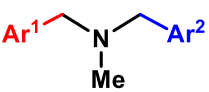

$$
\begin{aligned}
& \mathrm{Ar}^{1} \widehat{M e}^{\stackrel{\oplus}{N}} \widehat{A r}_{{ }^{\ominus}}{ }^{\ominus} \\
& \mathrm{Ar}^{1} \overbrace{\mathrm{NMe}_{3}}^{\mathrm{I}^{\ominus}} \overbrace{\mathrm{NMe}_{3}}^{\ominus}{ }^{\ominus} \\
& \text { entry } A-13 \\
& \text { entry } B-14 \\
& \text { entry } C-1 a+1 b
\end{aligned}
$$

$$
\begin{aligned}
& \mathrm{Ar}^{1} \overbrace{\mathrm{Br}} \text { at } \mathrm{Ar}^{1} \overbrace{\mathrm{Br}} \text { at } \\
& \mathrm{Ar}^{2} \smile \mathrm{Br}^{120^{\circ} \mathrm{C}} \quad \mathrm{Ar}^{2} \smile_{\mathrm{Br}} 23^{\circ} \mathrm{C} \\
& \text { entry } D-17 a+17 b \quad \text { entry } E-17 a+17 b
\end{aligned}
$$

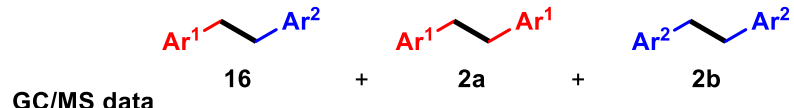

\begin{tabular}{|c|c|c|c|c|c|}
\hline entry & $\mathbf{1 6}$ & $\mathbf{2 a}$ & $\mathbf{2 b}$ & $\begin{array}{c}\text { Mass }^{[\mathrm{a}]} \\
(\mathrm{mg})\end{array}$ & Yield (\%) \\
\hline A & 104615308 & 23773586 & 110985998 & 38 & 45 \\
\hline B & 926854805 & 365736869 & 840914166 & 35 & 41 \\
\hline C & 97439385 & 31709432 & 80488582 & 40 & 46 \\
\hline D & 1694886768 & 986109388 & 1446191844 & 61 & 71 \\
\hline E & 2381165935 & 2002370065 & 155445586 & 75 & 87 \\
\hline
\end{tabular}

c) normalized product distributions ${ }^{[b]}$ :

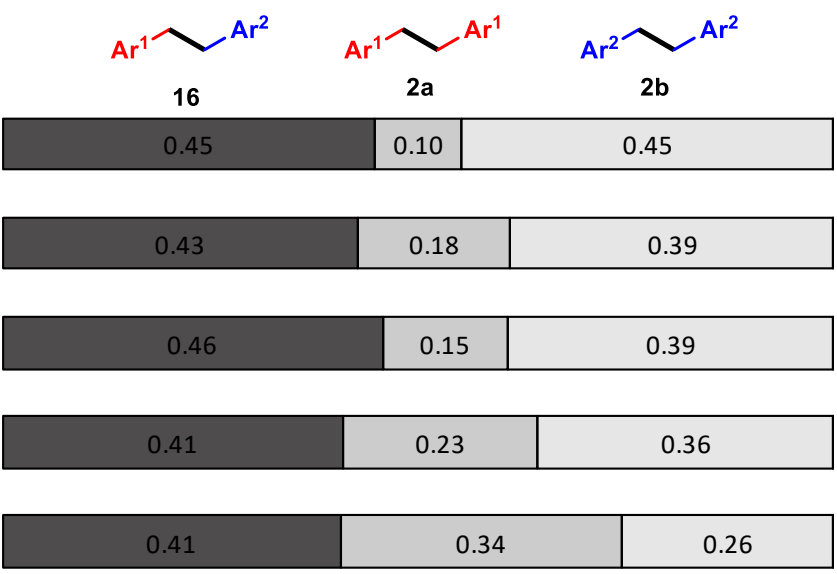

Fig. S5. GC/MS data showing product distribution

[a] Recovered mass and calculated combined yield following silica gel filtration

${ }^{[b]}$ Normalized sum $(\mathbf{1 6}+\mathbf{2 a}+\mathbf{2 b})=1$ 


\section{V. ${ }^{1} \mathrm{H}$ and ${ }^{13} \mathrm{C}$ NMR Spectra}

$400 \mathrm{MHz}, \mathrm{D}_{2} \mathrm{O}$<smiles>CN(C)Cc1ccccc1</smiles>

$1 \mathrm{a}$
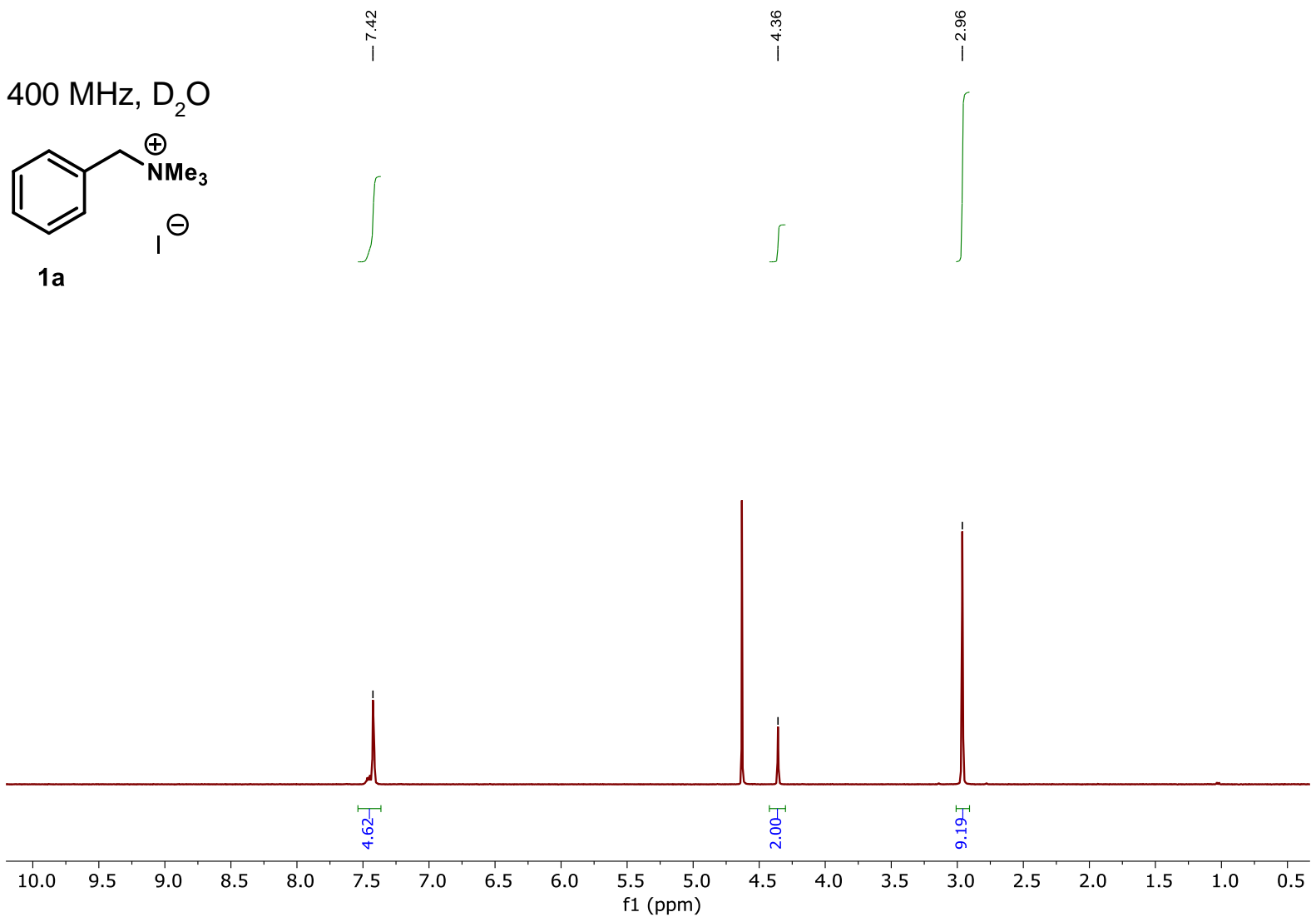

$125 \mathrm{MHz}, \mathrm{D}_{2} \mathrm{O}$<smiles>C[N+](=O)Cc1ccccc1</smiles>

1a

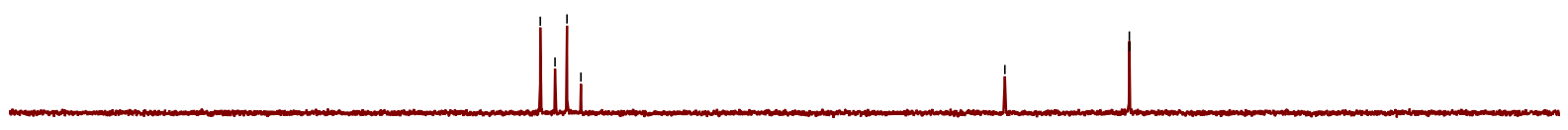

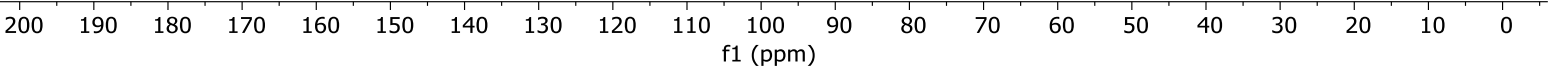




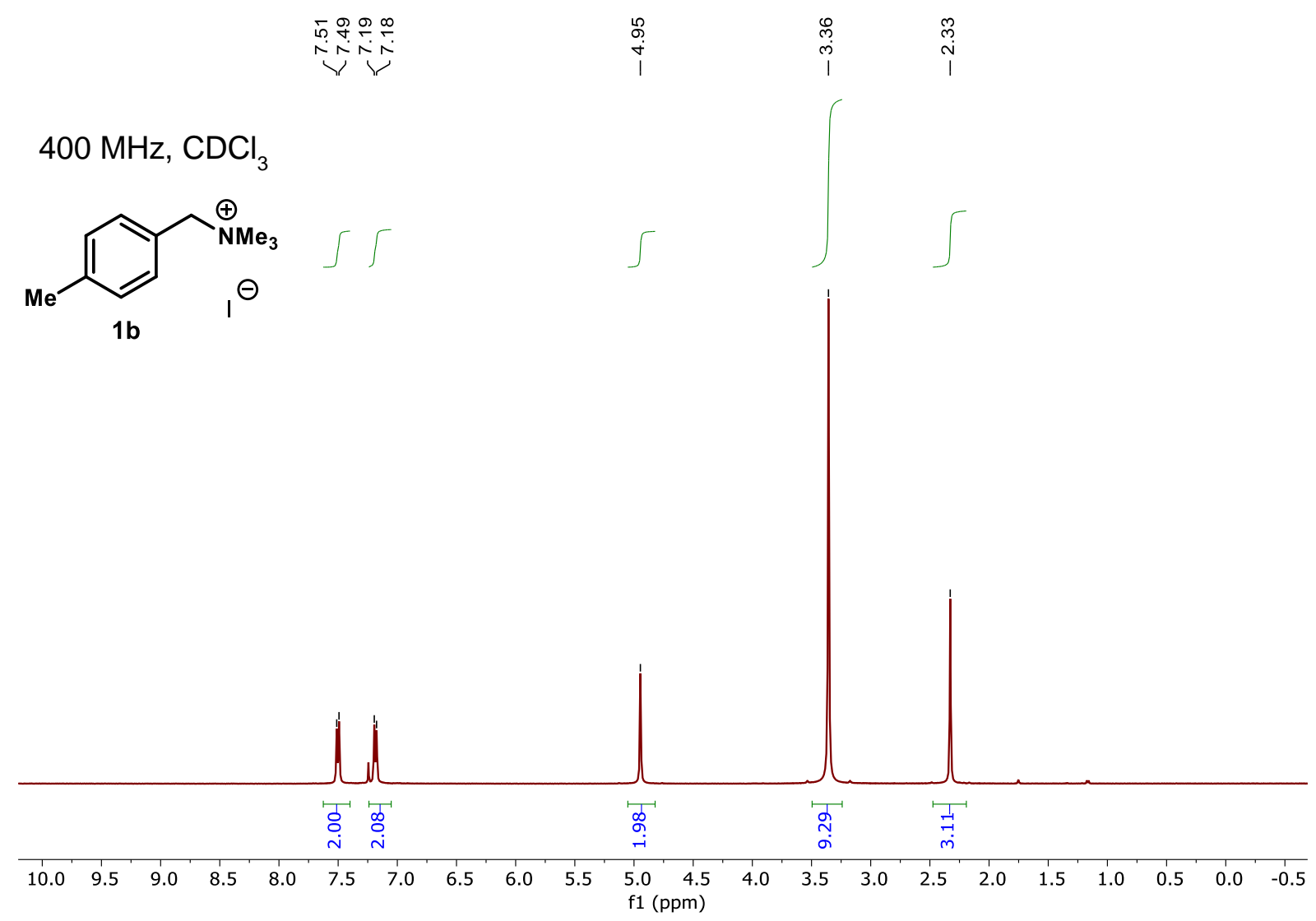

$125 \mathrm{MHz}, \mathrm{CDCl}_{3}$<smiles>Cc1ccc(CN(C)C)cc1</smiles>

1b

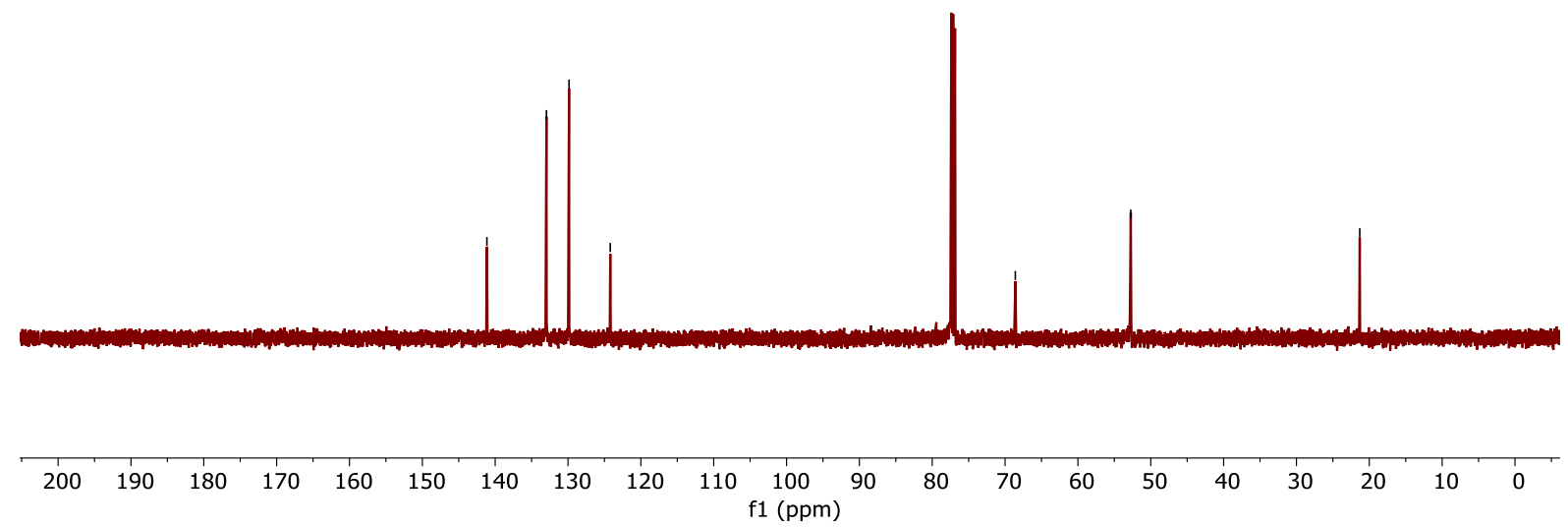




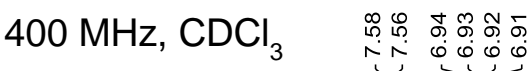

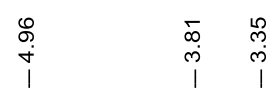<smiles>COc1ccc(CN(C)O)cc1</smiles>
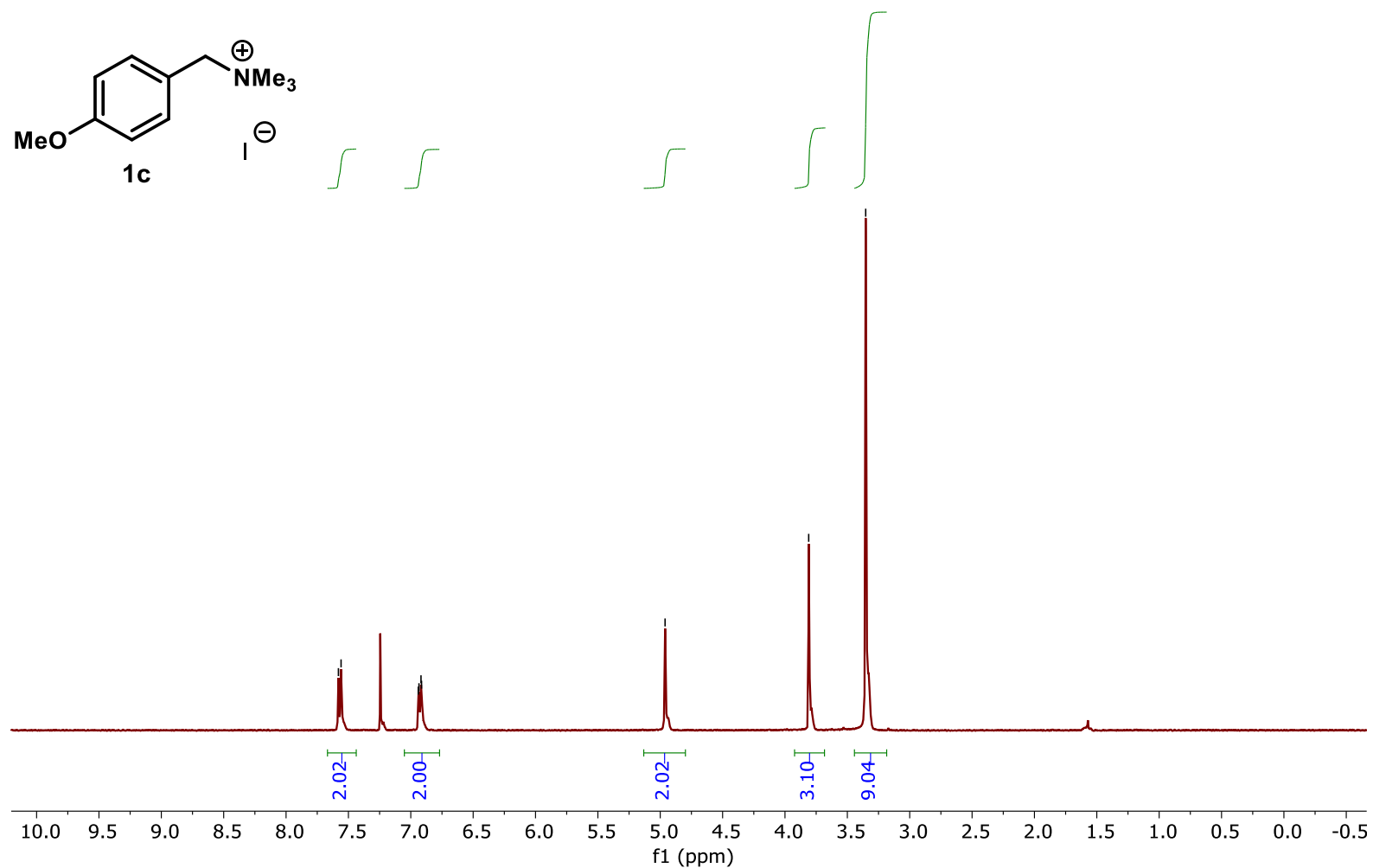

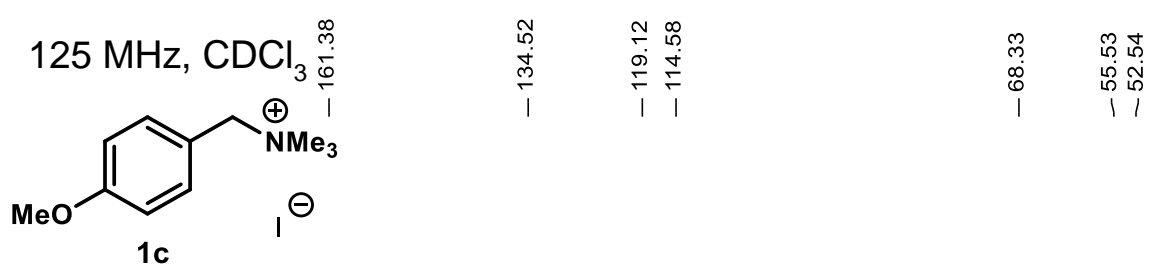

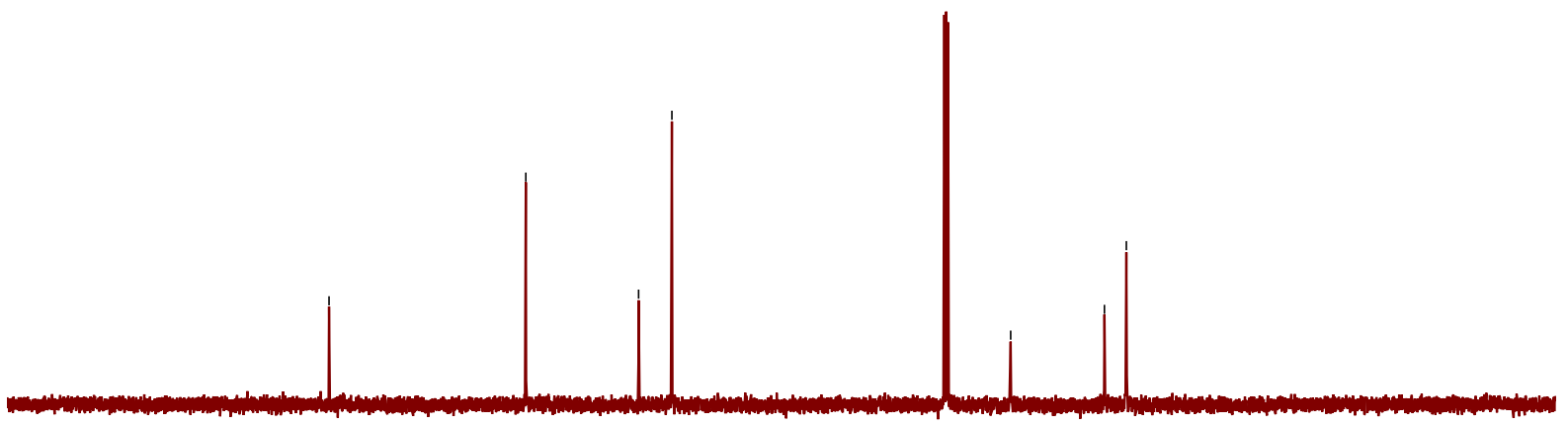

$\begin{array}{lllllllllllllllllllllll}200 & 190 & 180 & 170 & 160 & 150 & 140 & 130 & 120 & 110 & \begin{array}{c}100 \\ \mathrm{f} 1(\mathrm{ppm})\end{array} & 90 & 80 & 70 & 60 & 50 & 40 & 30 & 20 & 10 & 0\end{array}$ 
$500 \mathrm{MHz}, \mathrm{D}_{2} \mathrm{O}$<smiles>CN(CCO)Cc1ccc(F)cc1</smiles>
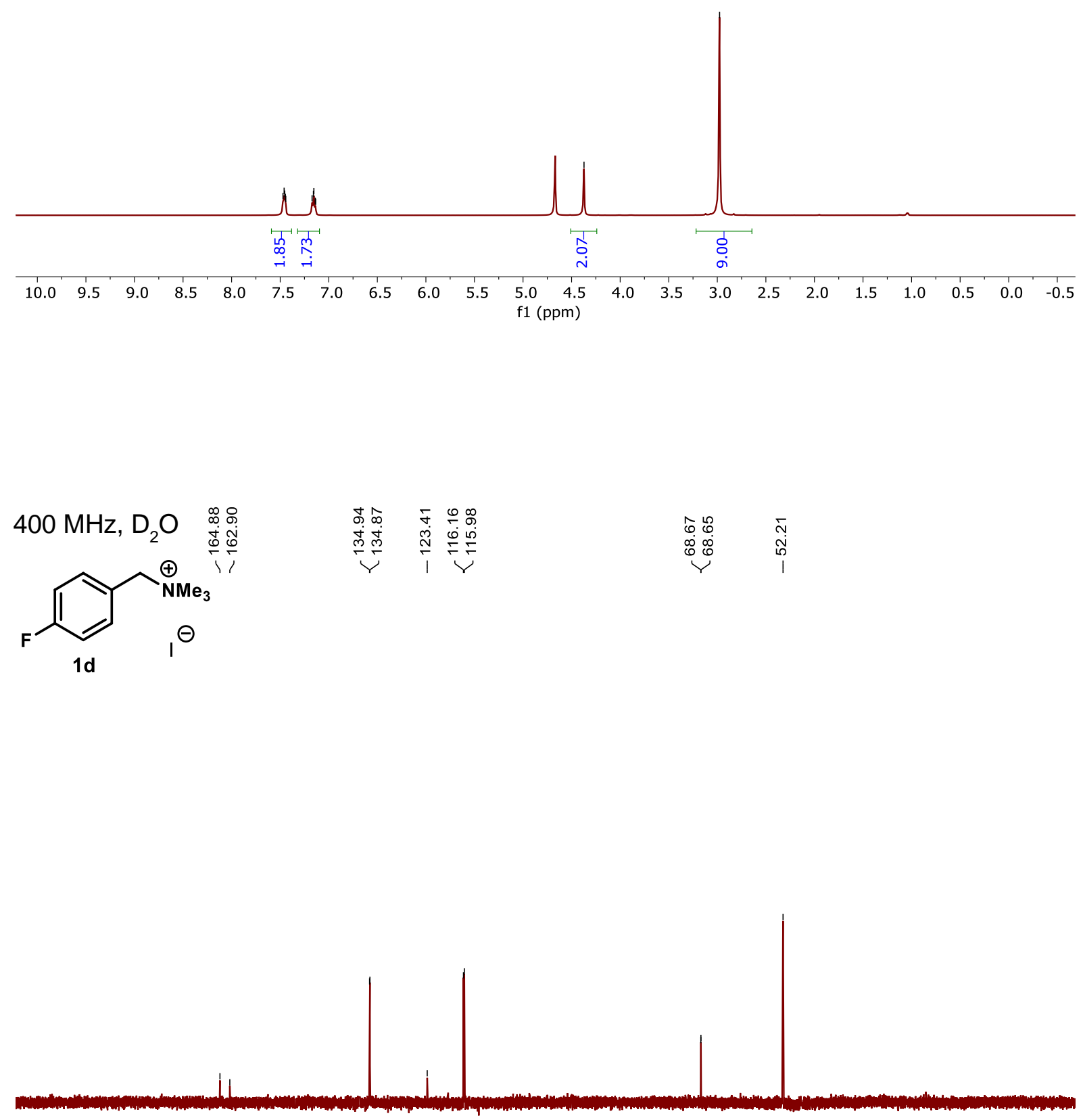

$\begin{array}{llllllllllllllllllllll}200 & 190 & 180 & 170 & 160 & 150 & 140 & 130 & 120 & 110 & \begin{array}{c}100 \\ \mathrm{f} 1(\mathrm{ppm})\end{array} & 90 & 80 & 70 & 60 & 50 & 40 & 30 & 20 & 10 & 0\end{array}$ 
$500 \mathrm{MHz} \mathrm{CDCl}_{3}$<smiles>Cc1cccc(CN(C)C)c1</smiles><smiles>C1=CCCCCC1</smiles>

$1 e$

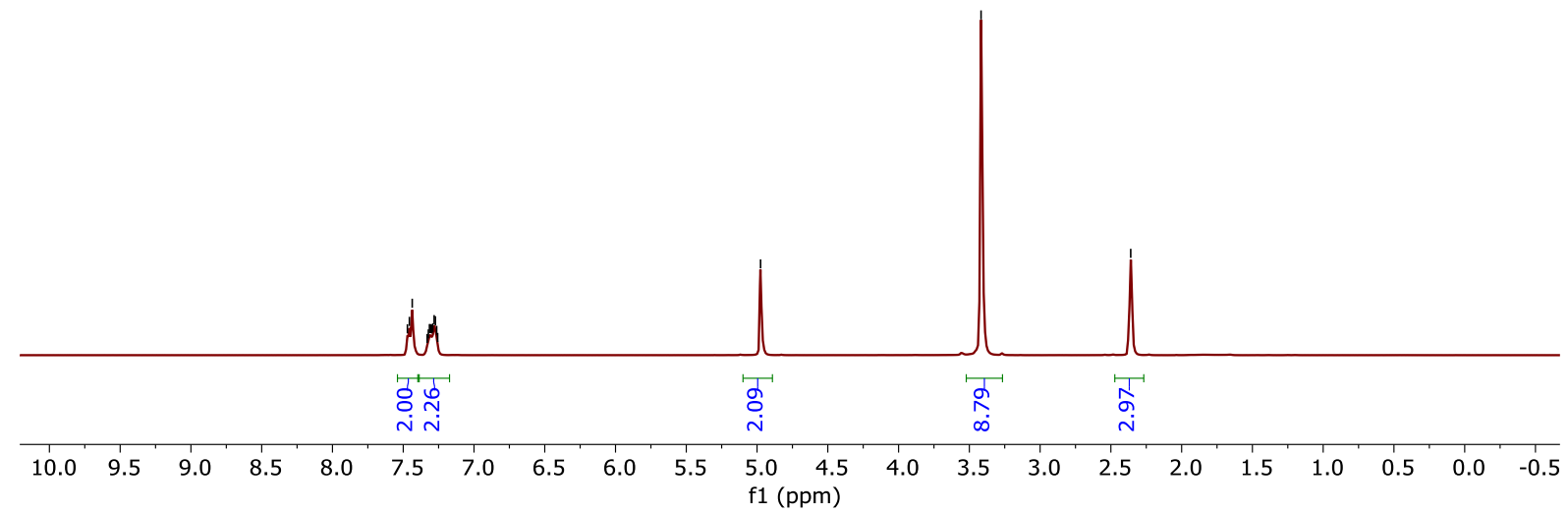

$125 \mathrm{MHz}, \mathrm{CDCl}_{3}$<smiles>Cc1cccc(CN(C)C)c1</smiles>
ᄀำ

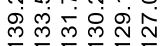
पi

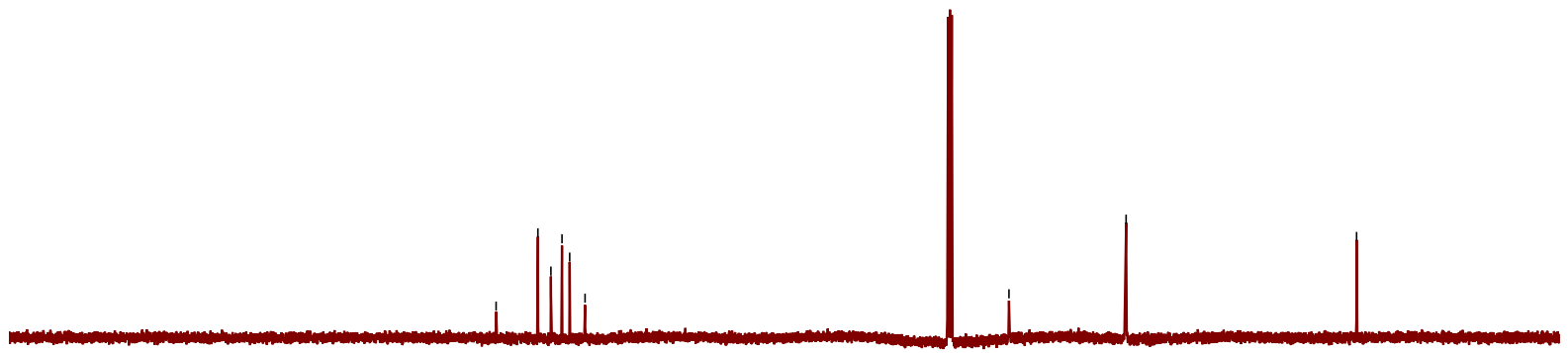

$\begin{array}{llllllllllllllllllllll}200 & 190 & 180 & 170 & 160 & 150 & 140 & 130 & 120 & 110 & 100 & 90 & 80 & 70 & 60 & 50 & 40 & 30 & 20 & 10 & 0\end{array}$ 
$500 \mathrm{MHz}^{\mathrm{CDCl}_{3}}$<smiles>COc1cccc(CN(C)C)c1</smiles>

If

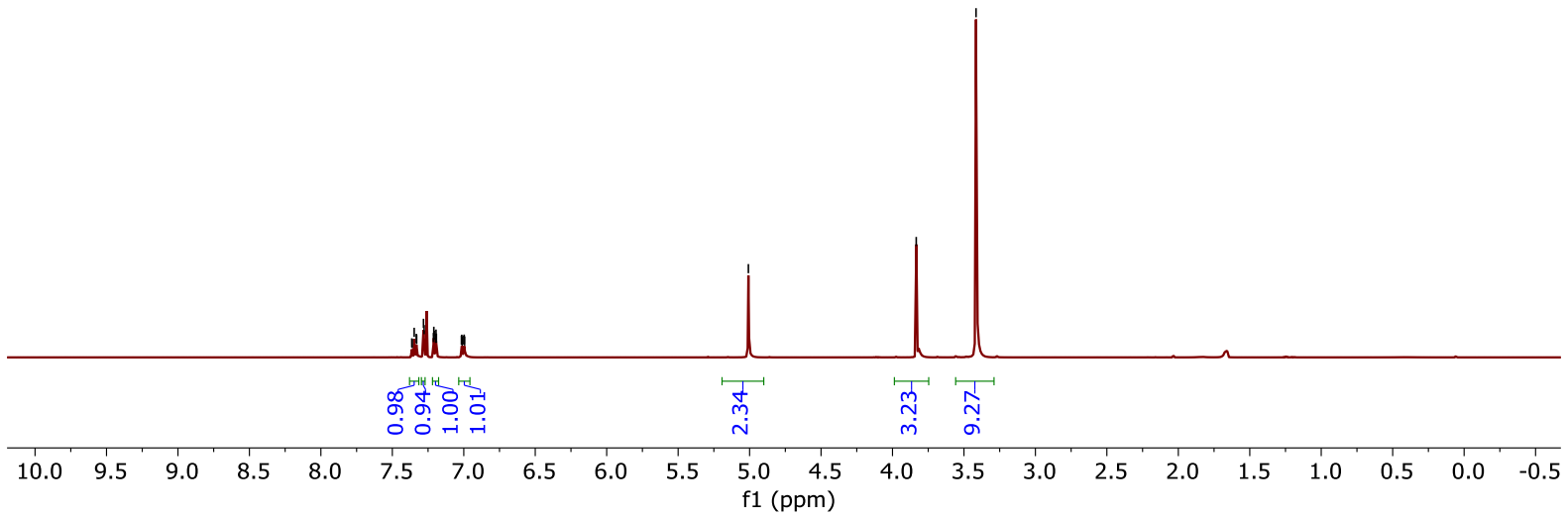

$125 \mathrm{MHz}, \mathrm{CDCl}_{3} \stackrel{\stackrel{\circ}{\circ}}{\leftarrow}$

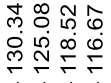

$\begin{array}{lll}\infty & 0 & \infty \\ \infty & 0 & 0 \\ \infty & 0 & 0 \\ 0 & 0 & 0 \\ 0 & 1 & 1\end{array}$<smiles>COc1cc[c]c(CN(C)O)c1</smiles>

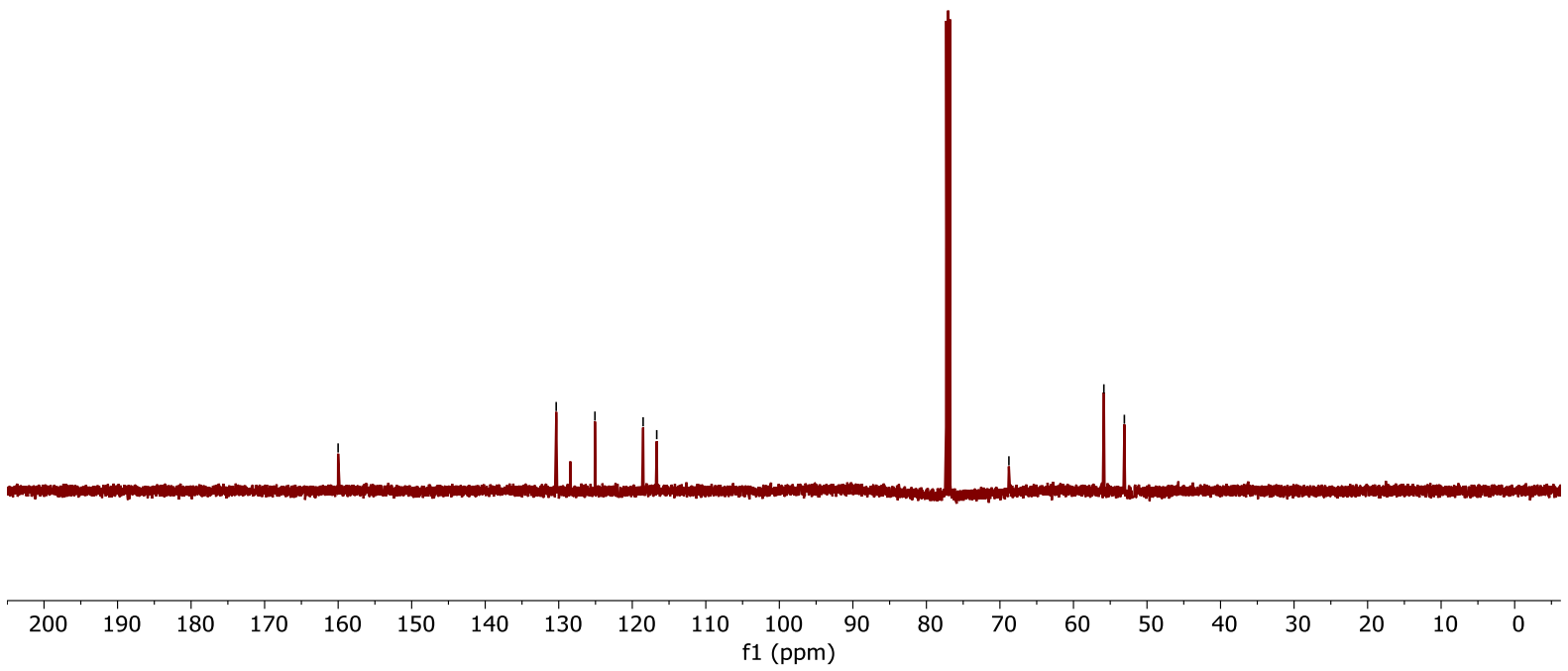


$400 \mathrm{MHz} \mathrm{CDCl}_{3}$<smiles>CN(C)Cc1cccc(F)c1</smiles>
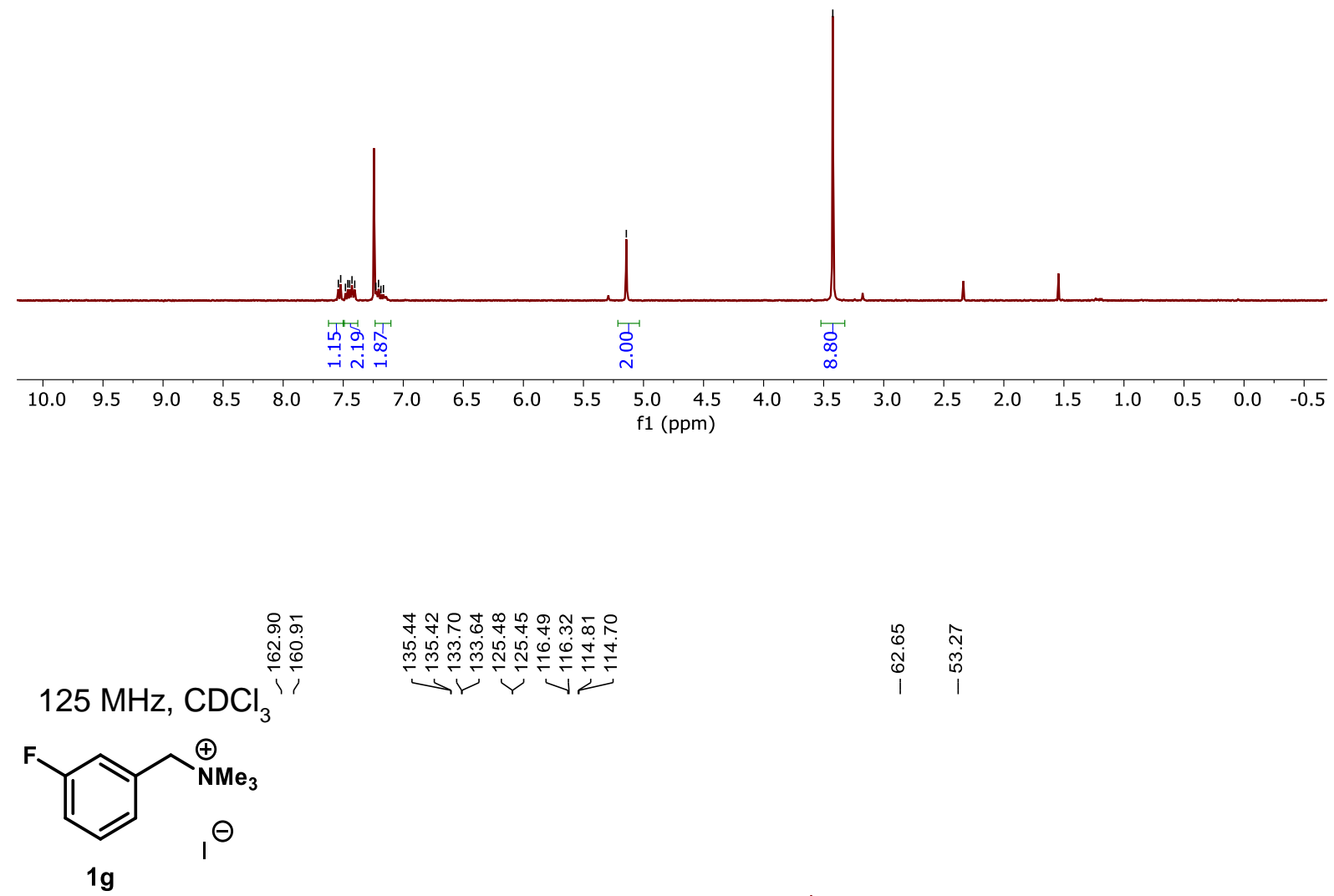

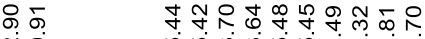

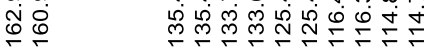

$\mathrm{Cl}_{3}$

$\underbrace{-V}_{V}-\bar{v}$

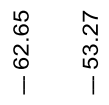

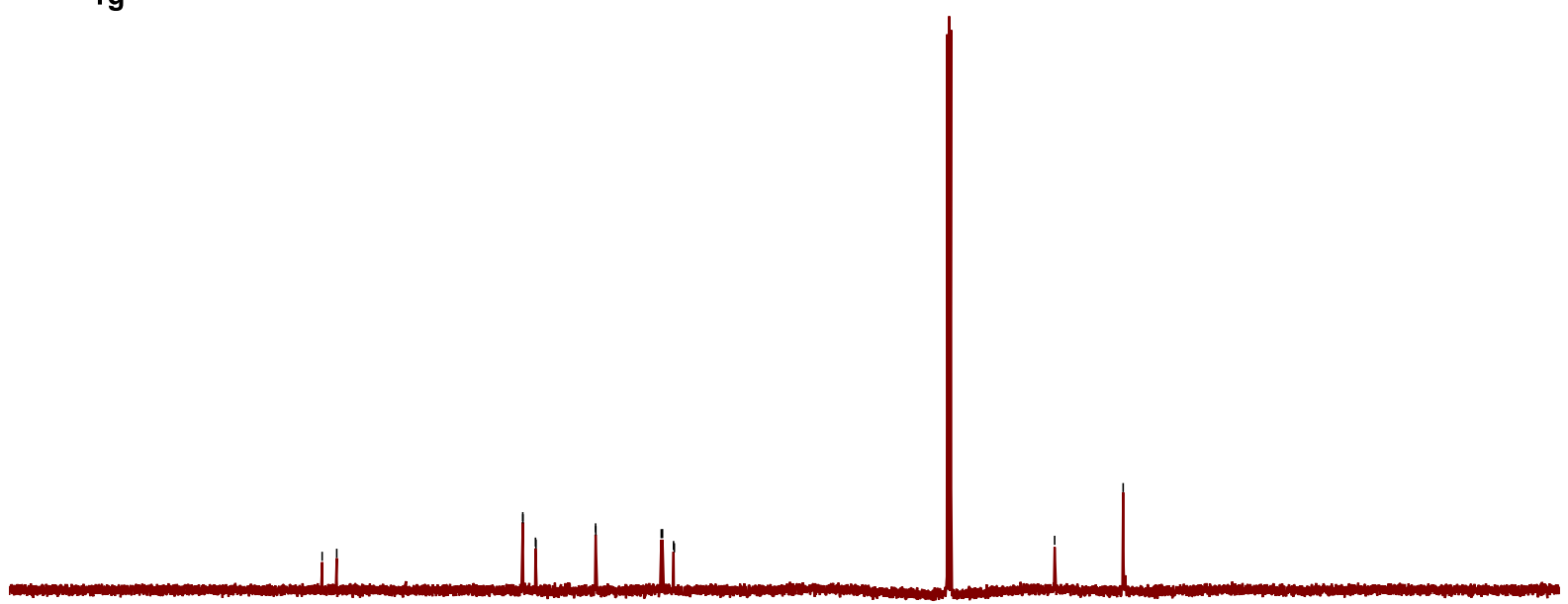

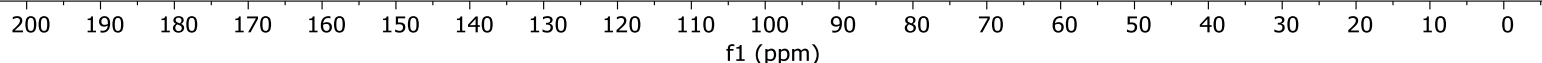



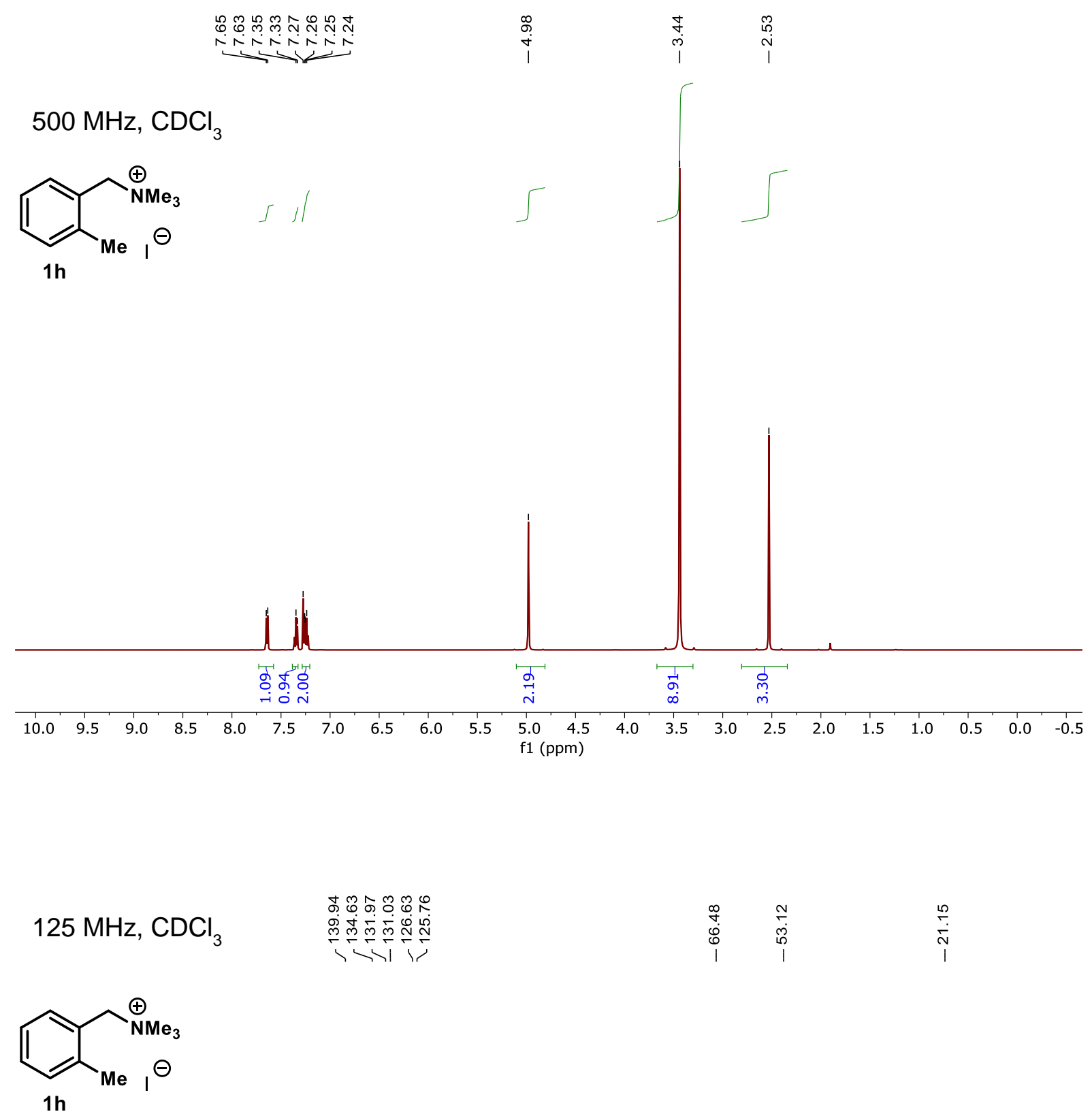

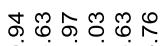

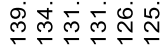

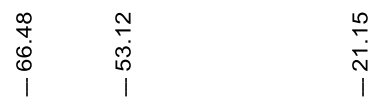

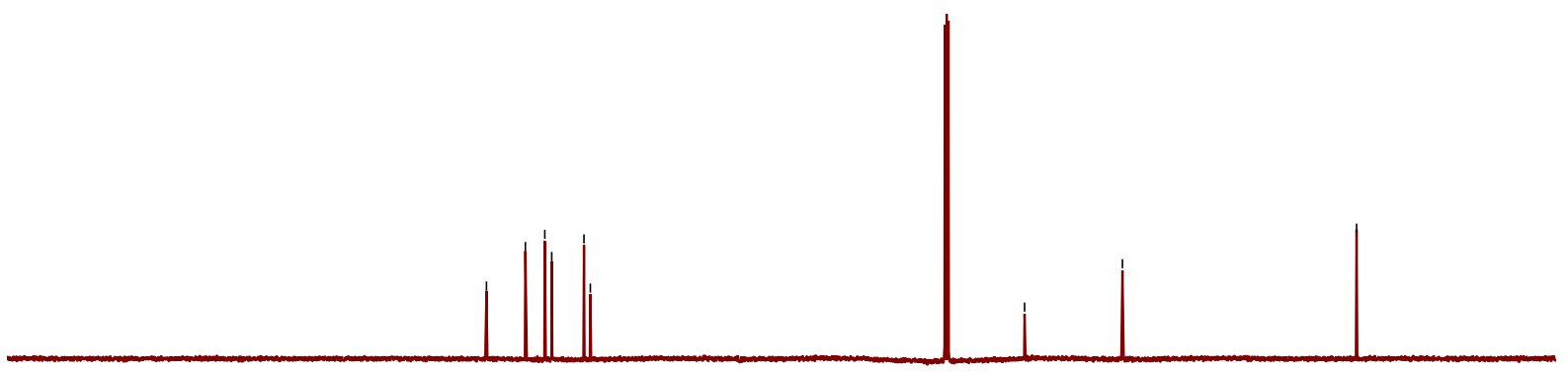

$\begin{array}{lllllllllllllllllllll}200 & 190 & 180 & 170 & 160 & 150 & 140 & 130 & 120 & 110 & \begin{array}{c}100 \\ \mathrm{f} 1(\mathrm{ppm})\end{array} & 90 & 80 & 70 & 60 & 50 & 40 & 30 & 20 & 10 & 0\end{array}$ 
$500 \mathrm{MHz}, \mathrm{CDCl}_{3}$<smiles>COc1ccccc1CN(C)C</smiles>

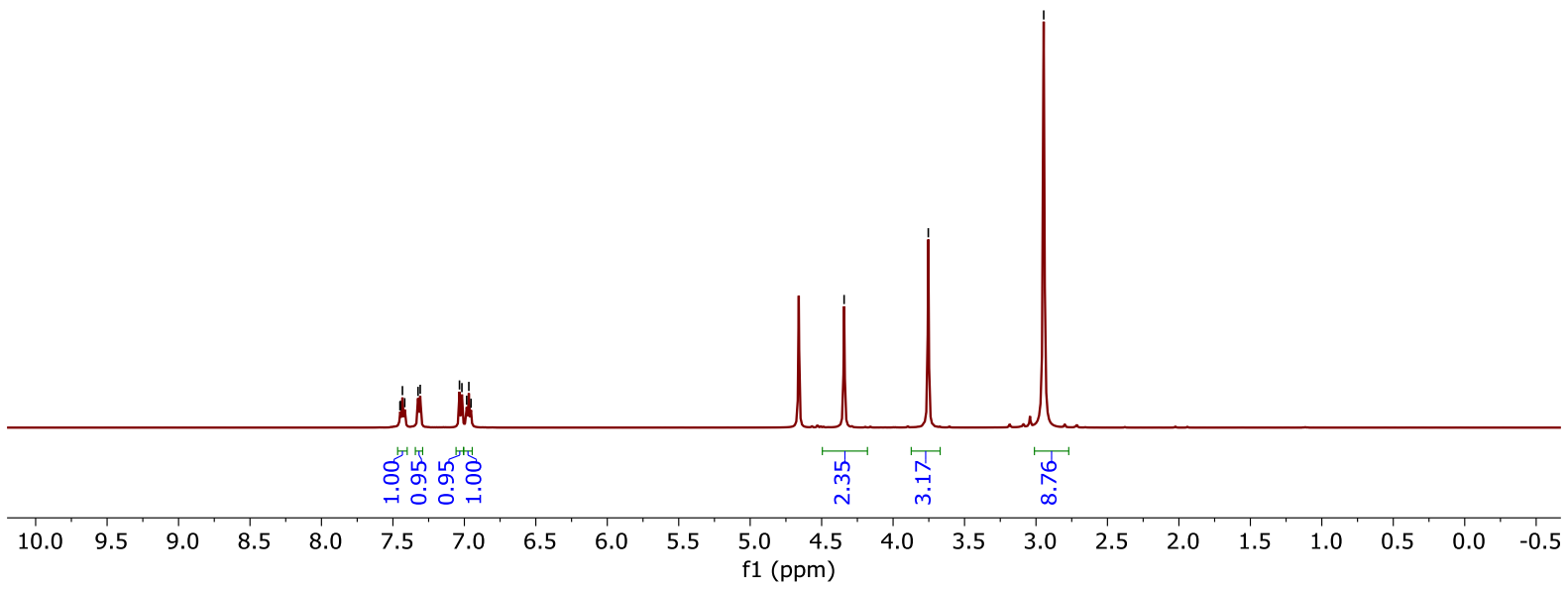

$125 \mathrm{MHz}, \mathrm{CD}$

1i

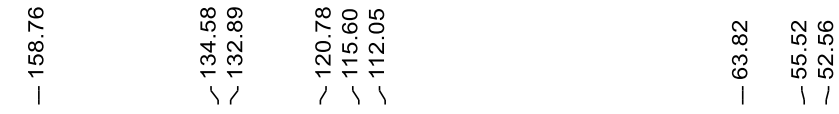

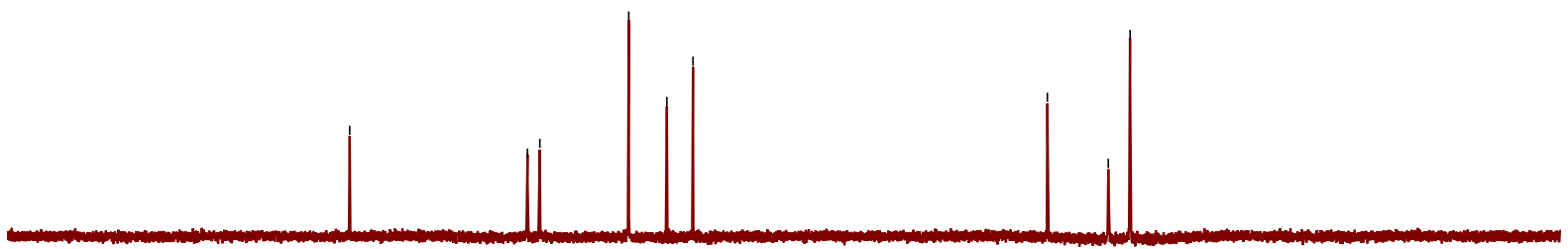

\begin{tabular}{llllllllllllllllllllll}
\hline 200 & 190 & 180 & 170 & 160 & 150 & 140 & 130 & 120 & 110 & 100 & 90 & 80 & 70 & 60 & 50 & 40 & 30 & 20 & 10 & 0 \\
$\mathrm{f} 1(\mathrm{ppm})$ &
\end{tabular} 
$500 \mathrm{MHz}, \mathrm{CDCl}_{3}$<smiles>CN(Cc1ccccc1)Cc1ccccc1F</smiles>

1j

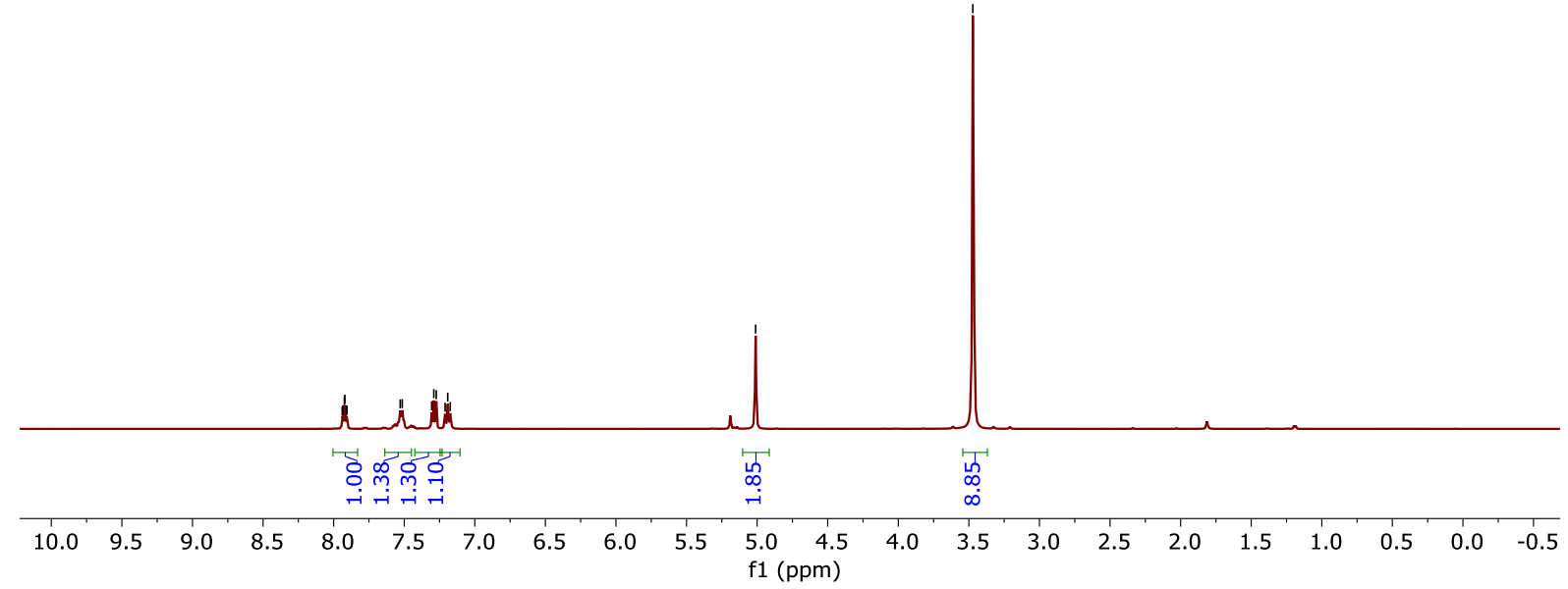

$125 \mathrm{MHz}, \mathrm{CD}$

1j

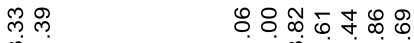

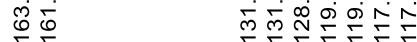

VIV VIF

$\begin{array}{lll}0 & \overline{0} \\ \infty & \stackrel{0}{0} \\ 0 & 0 \\ 0 & 1 & 1\end{array}$

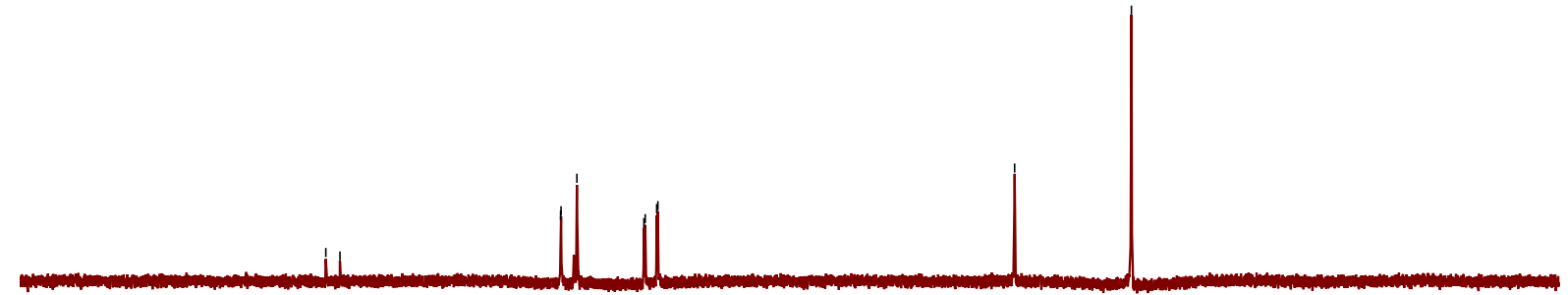

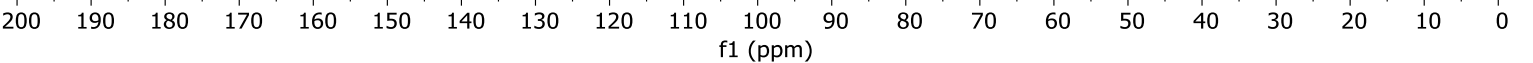


$400 \mathrm{MHz}, \mathrm{D}_{2} \mathrm{O}$

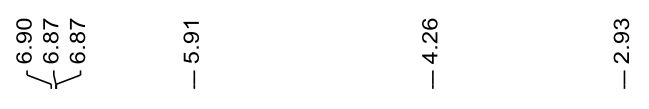
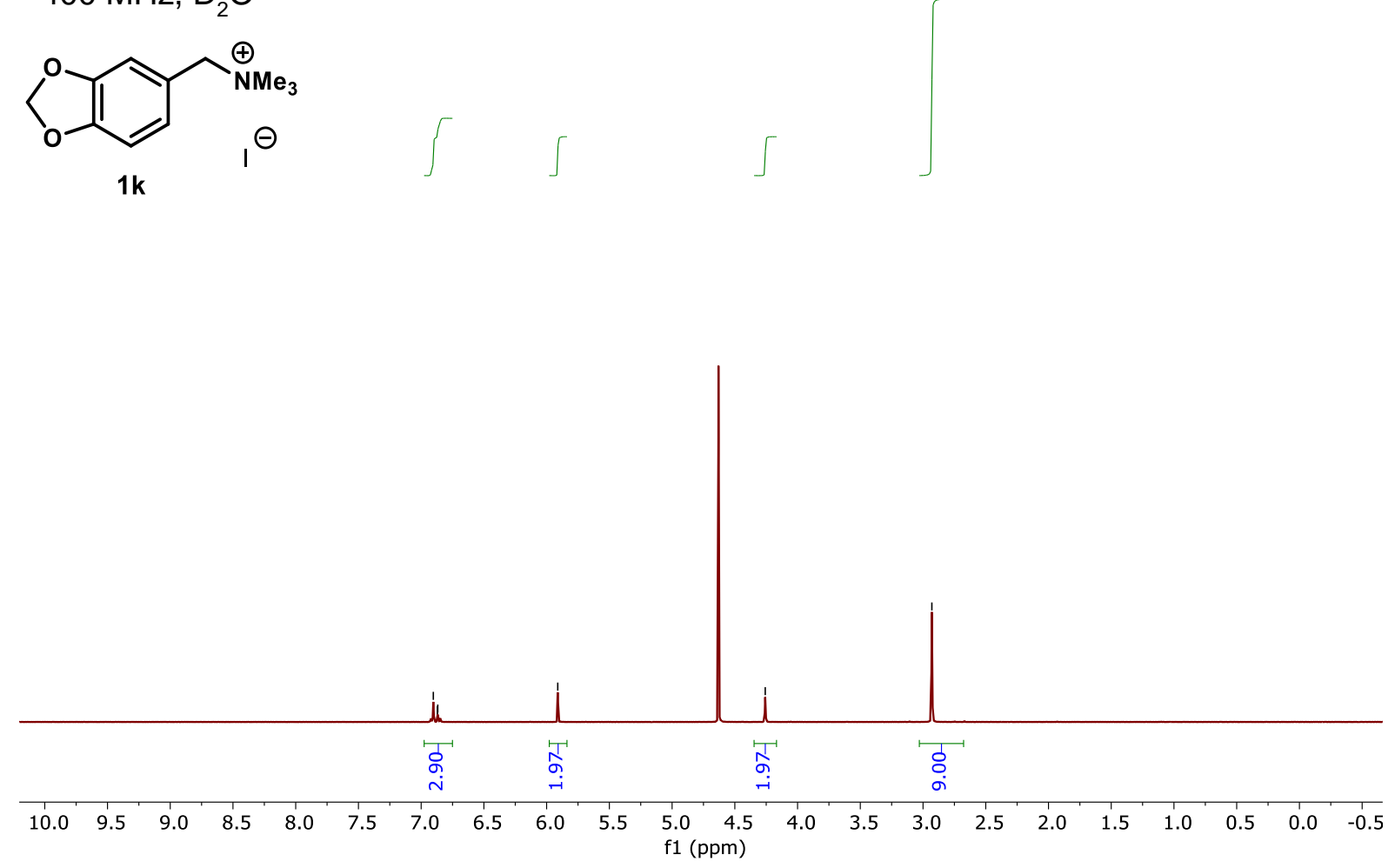

\begin{tabular}{|c|c|c|c|}
\hline 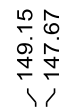 & 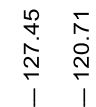 & 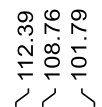 & $\begin{array}{l}\text { के } \\
\ddot{8} \\
\dot{0} \\
1\end{array}$ \\
\hline
\end{tabular}

$125 \mathrm{MHz}, \mathrm{D}_{2} \mathrm{O}$<smiles>CN(C)Cc1ccc2c(c1)OCO2</smiles>

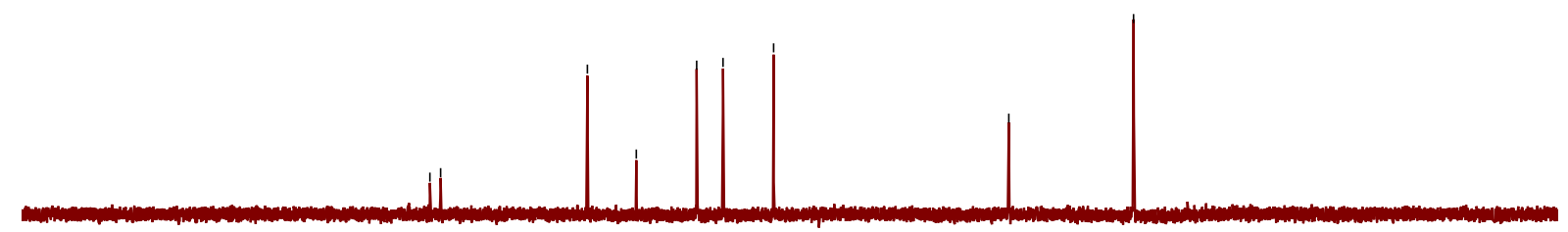

$\begin{array}{lllllllllllllllllllll}200 & 190 & 180 & 170 & 160 & 150 & 140 & 130 & 120 & 110 & \begin{array}{c}100 \\ \mathrm{f} 1(\mathrm{ppm})\end{array} & 90 & 80 & 70 & 60 & 50 & 40 & 30 & 20 & 10 & 0\end{array}$ 

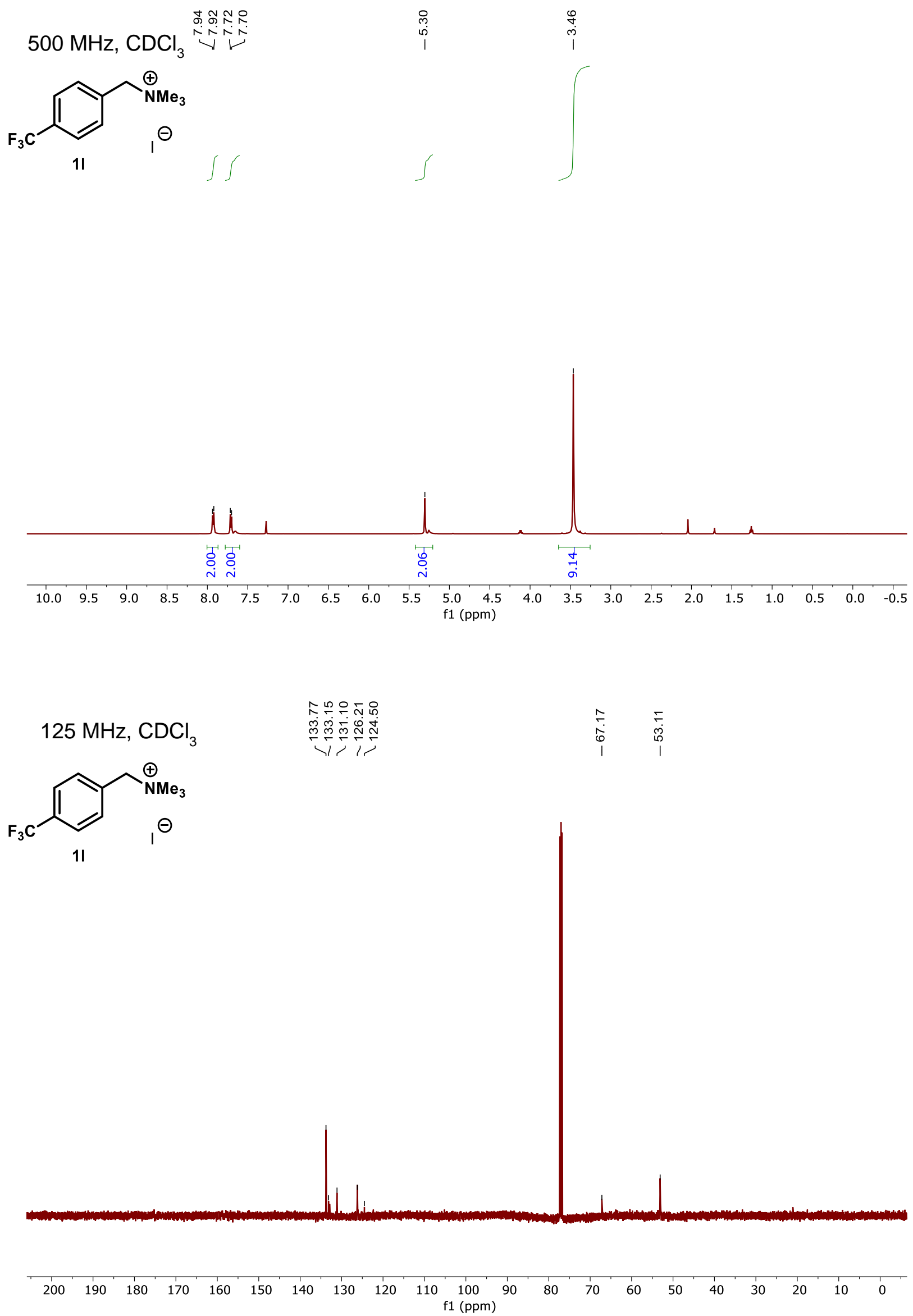

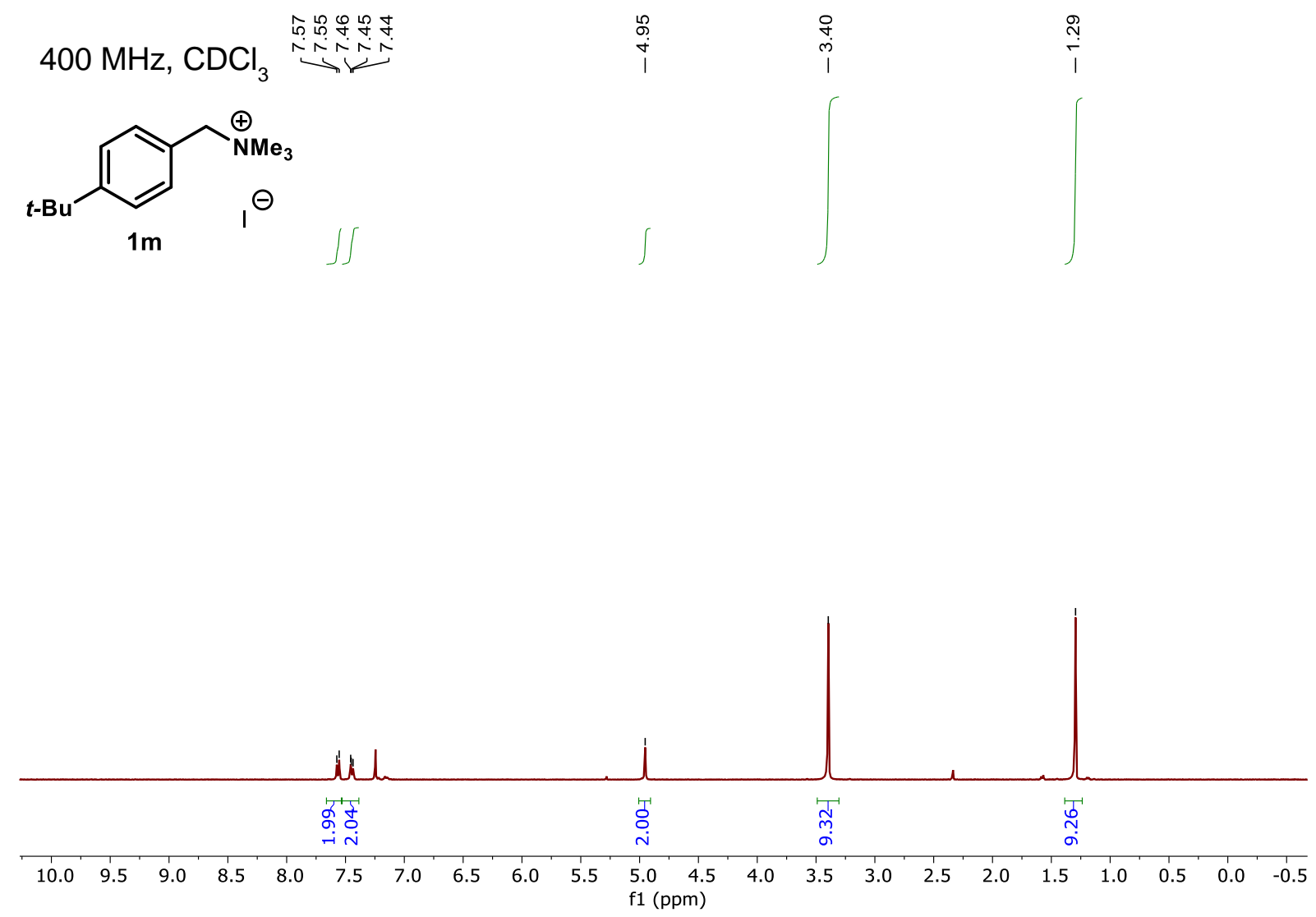

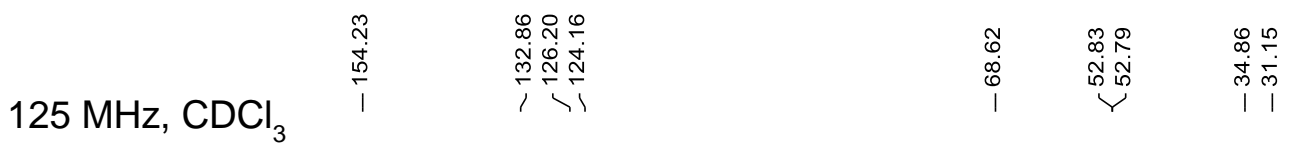<smiles>CCN(C)Cc1ccc(C(C)(C)C)cc1</smiles>

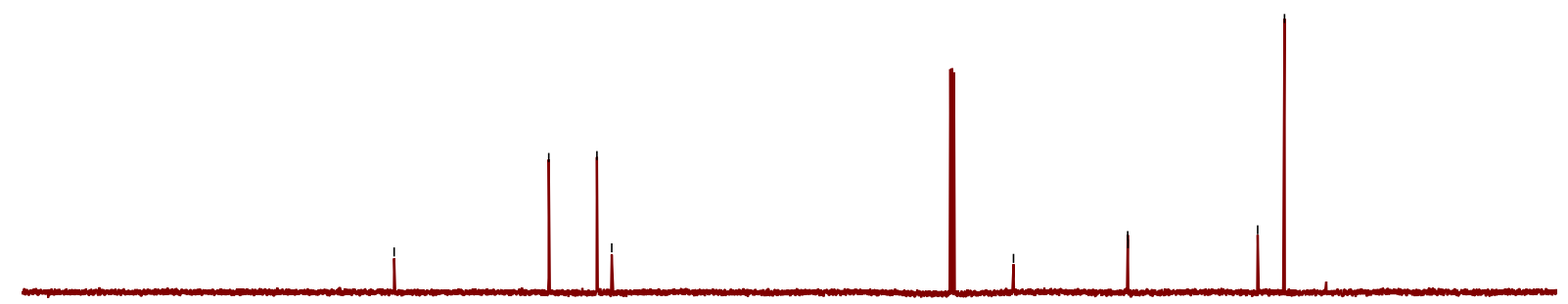

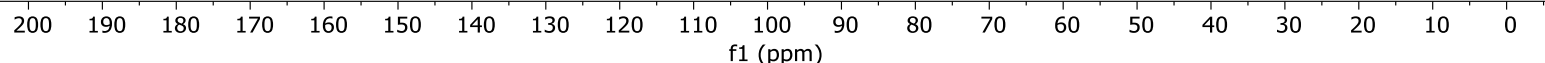


$500 \mathrm{MHz}, \mathrm{CDCl}_{3}$<smiles>C[N+](C)Cc1ccc2ccccc2c1</smiles>

1n

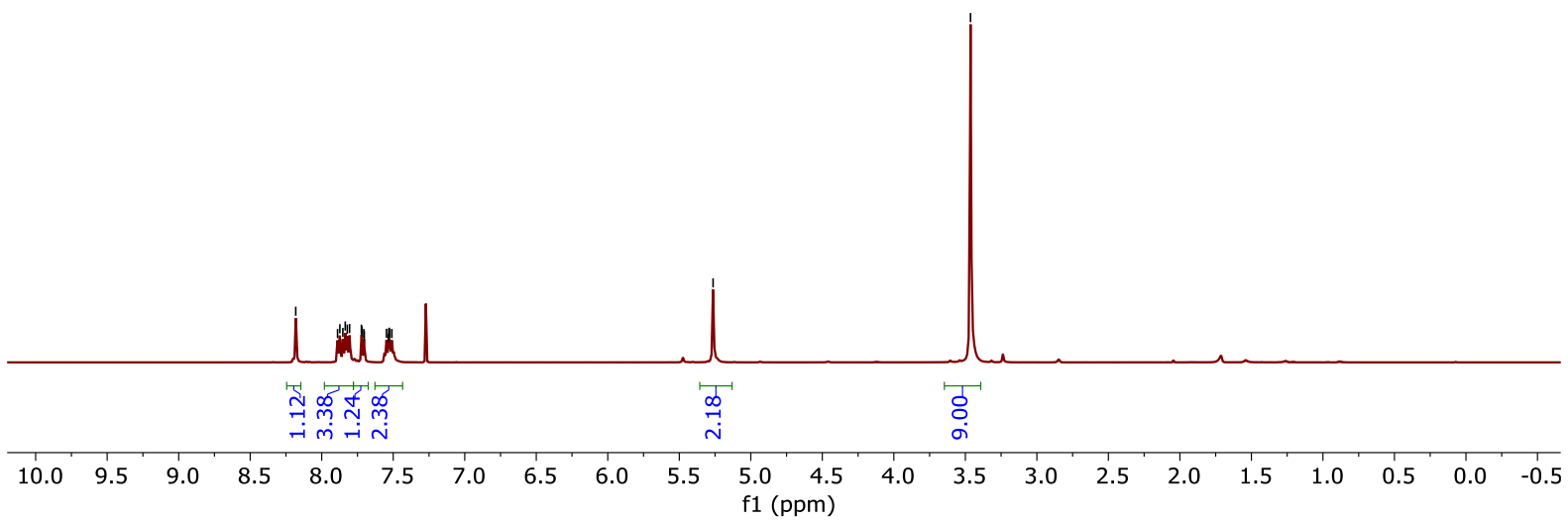

$125 \mathrm{MHz} \mathrm{CDCl}_{3}$<smiles>CN(C)Cc1ccc2ccccc2c1</smiles>

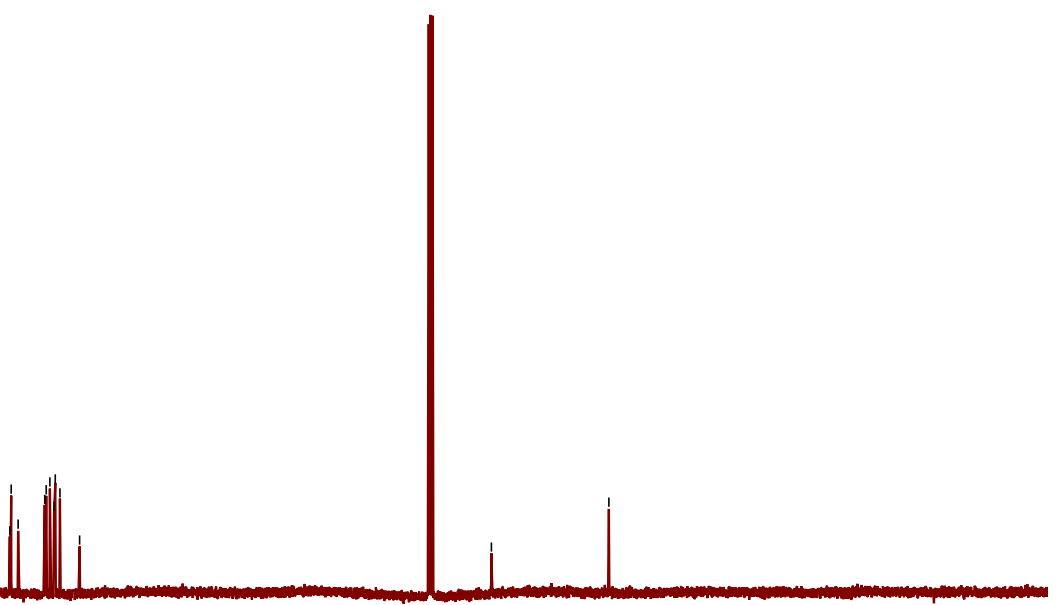

$\begin{array}{lllllllllllllllllllllll}200 & 190 & 180 & 170 & 160 & 150 & 140 & 130 & 120 & 110 & \begin{array}{c}100 \\ \mathrm{f} 1(\mathrm{ppm})\end{array} & 90 & 80 & 70 & 60 & 50 & 40 & 30 & 20 & 10 & 0\end{array}$ 
$500 \mathrm{MHz}, \mathrm{DMSO}-d_{6}$<smiles>O=[N+]([O-])Cc1ccc2ccccc2c1</smiles>

10

i

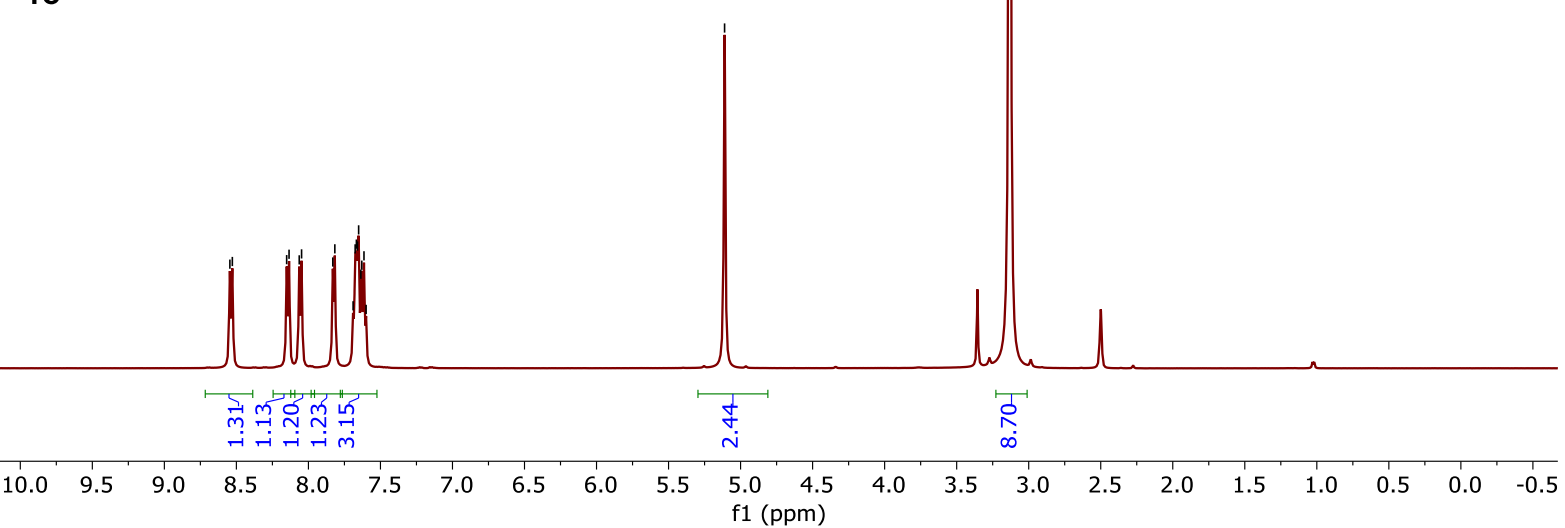
$125 \mathrm{MHz}$, DMSO-d<smiles>O=[N+]([O-])Cc1ccc2ccccc2c1</smiles>

$\oplus \oplus$ MTin

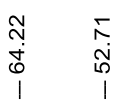

10

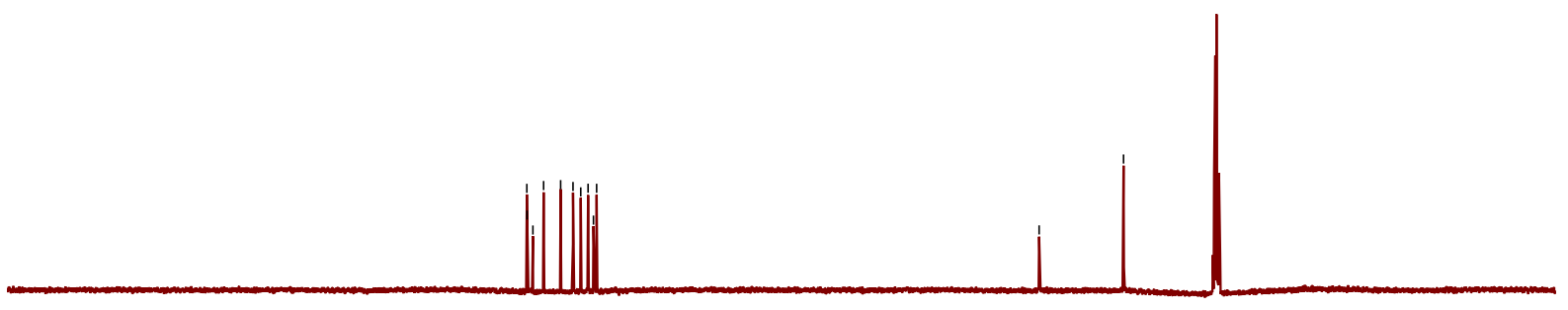

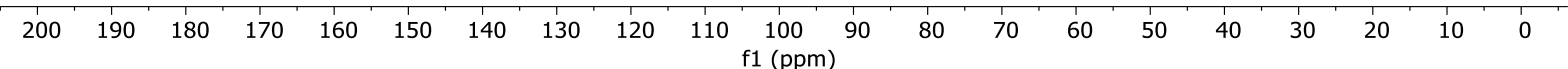




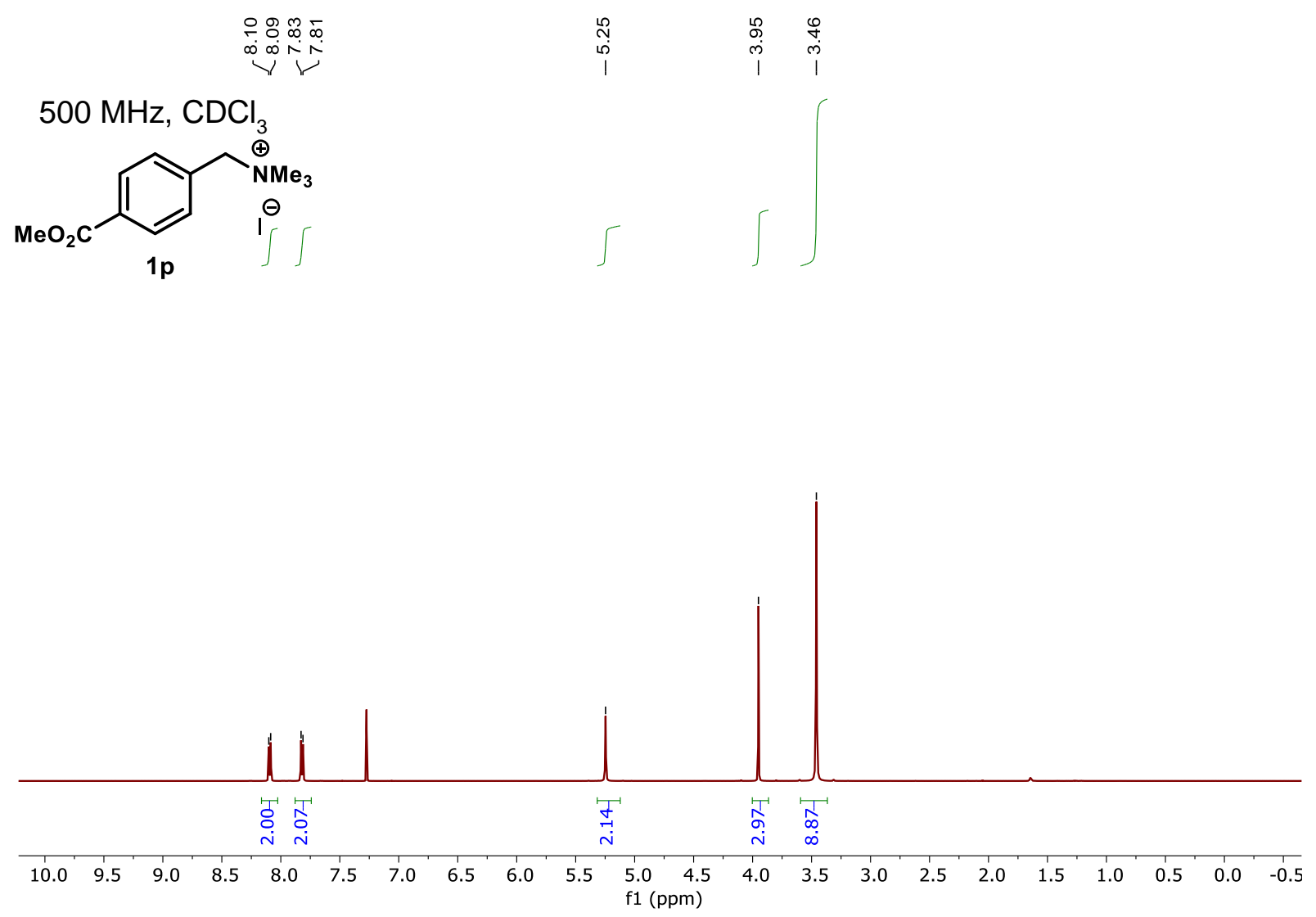

$125 \mathrm{MHz}, \mathrm{CDCl}_{3}^{\stackrel{a}{\mid}}$

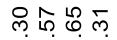

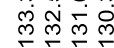

霍

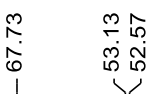<smiles>CC(=O)c1ccc(C[18O][Na])cc1</smiles>

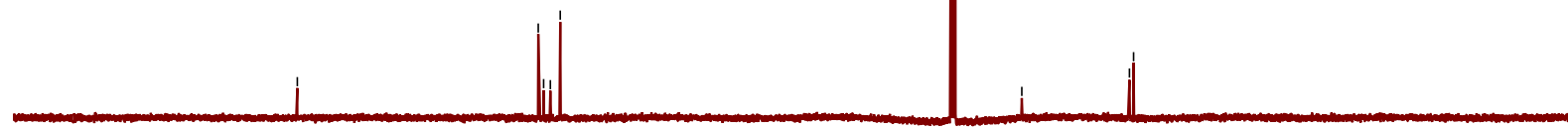

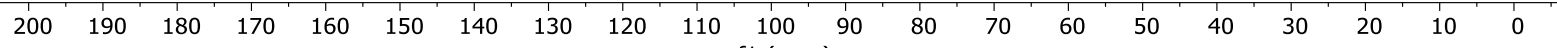




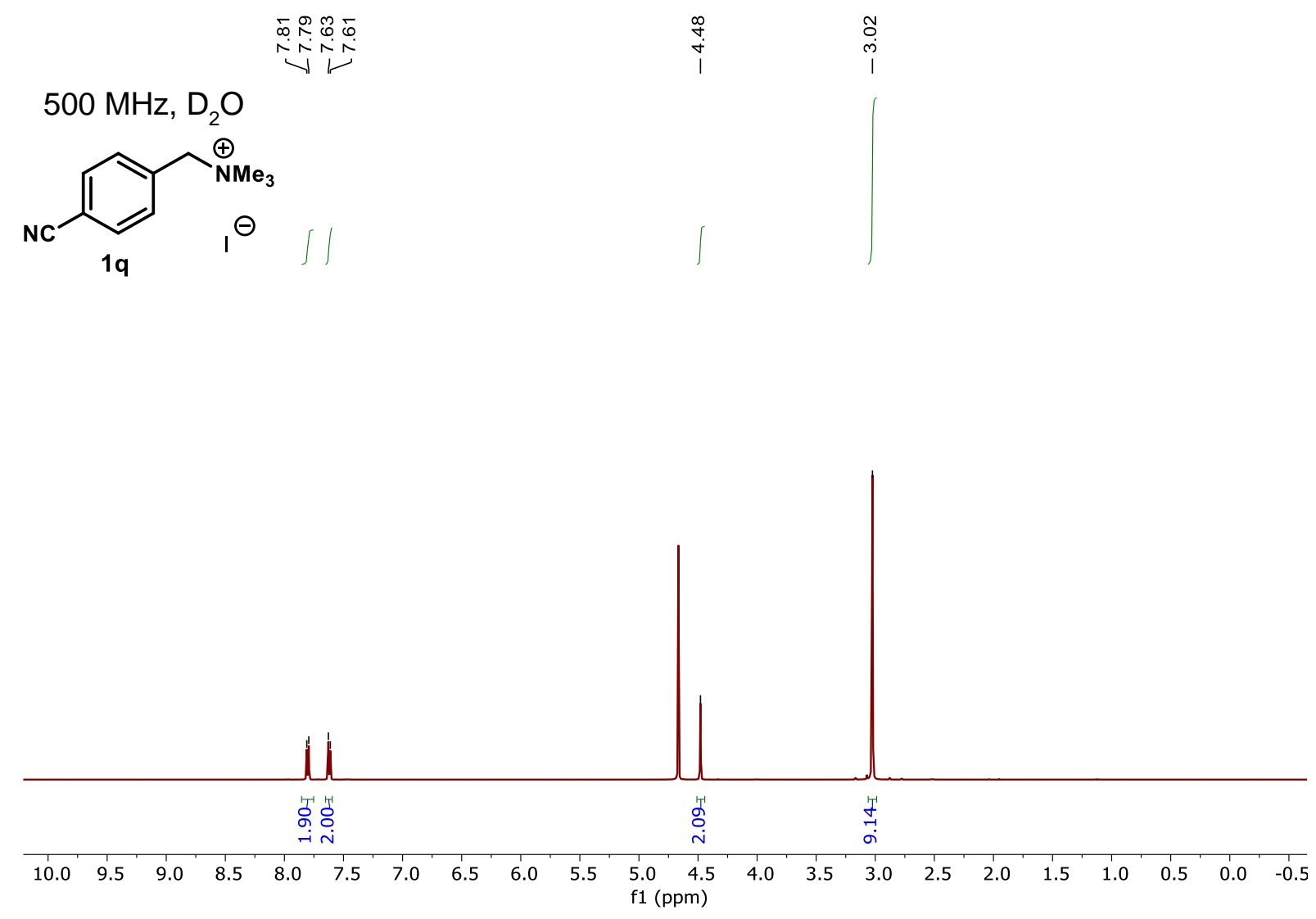

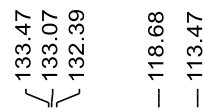

$\begin{array}{ll}m & 0 \\ 00 & 0 \\ 0 & \text { กี } \\ 0 & 1\end{array}$

$125 \mathrm{MHz}, \mathrm{D}_{2} \mathrm{O}$<smiles>N#Cc1ccc(CO[18O])cc1</smiles>

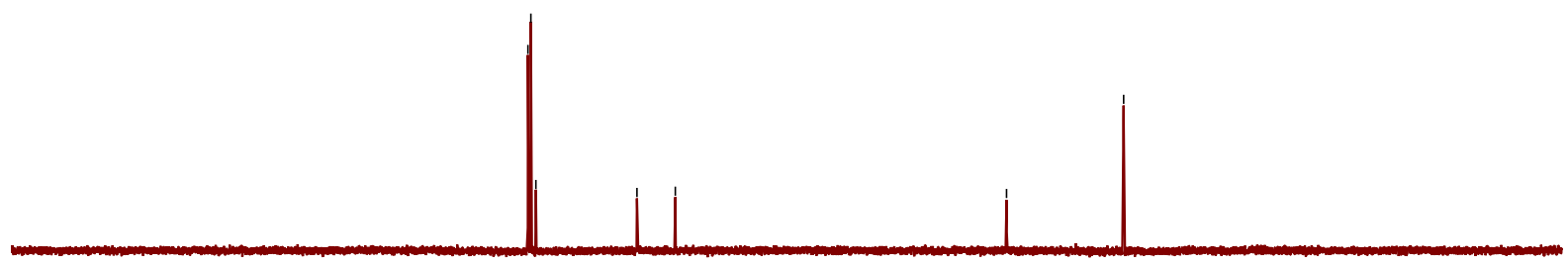

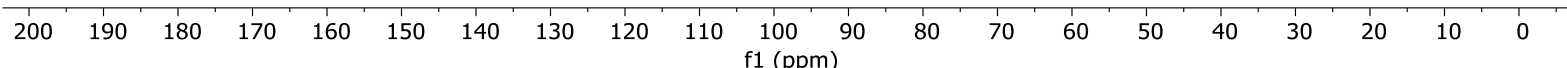




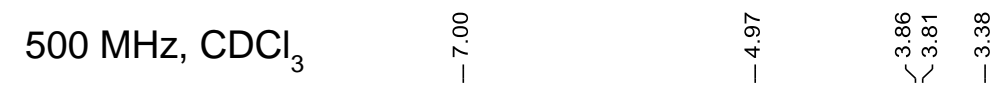<smiles>COc1cc(CN(C)C)cc(OC)c1OC</smiles>

$1 \mathrm{r}$

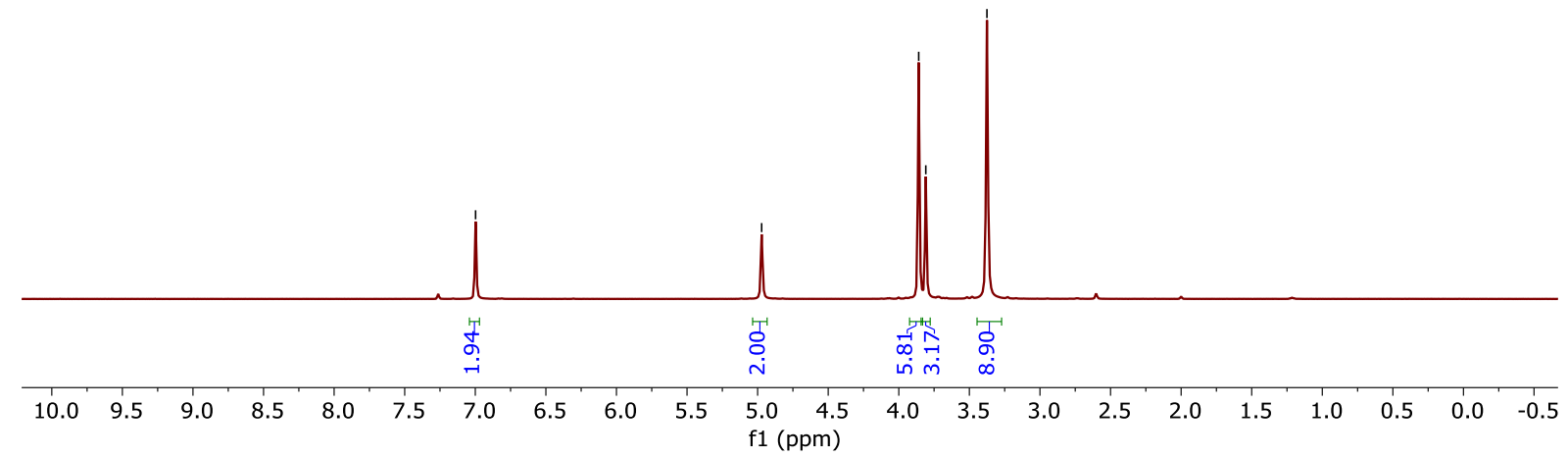

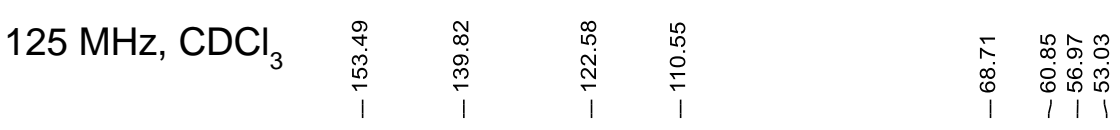<smiles>COc1cc(CN(C)C)cc(OC)c1OC</smiles>

$1 \mathrm{r}$

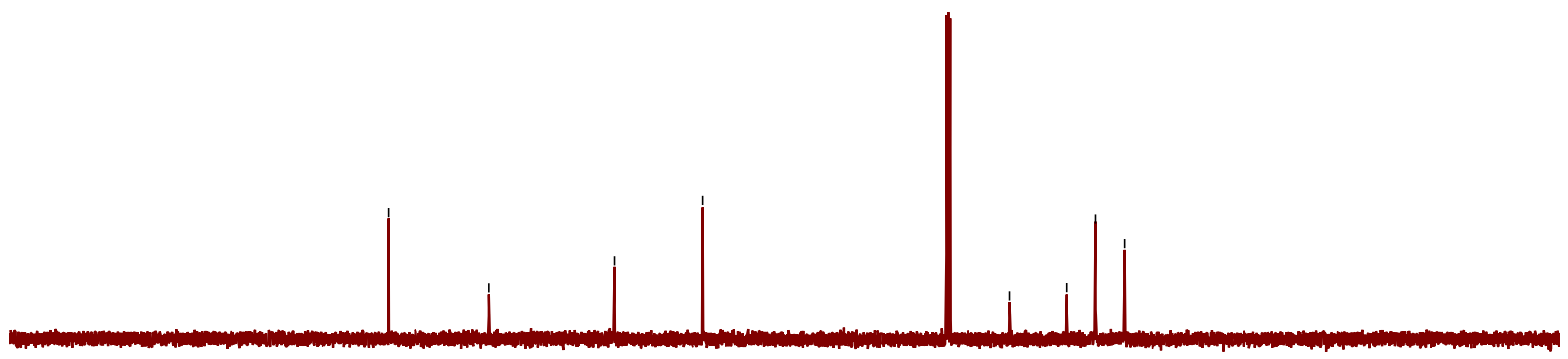

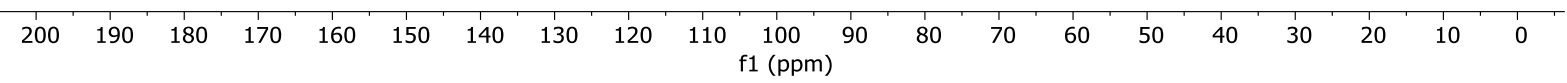




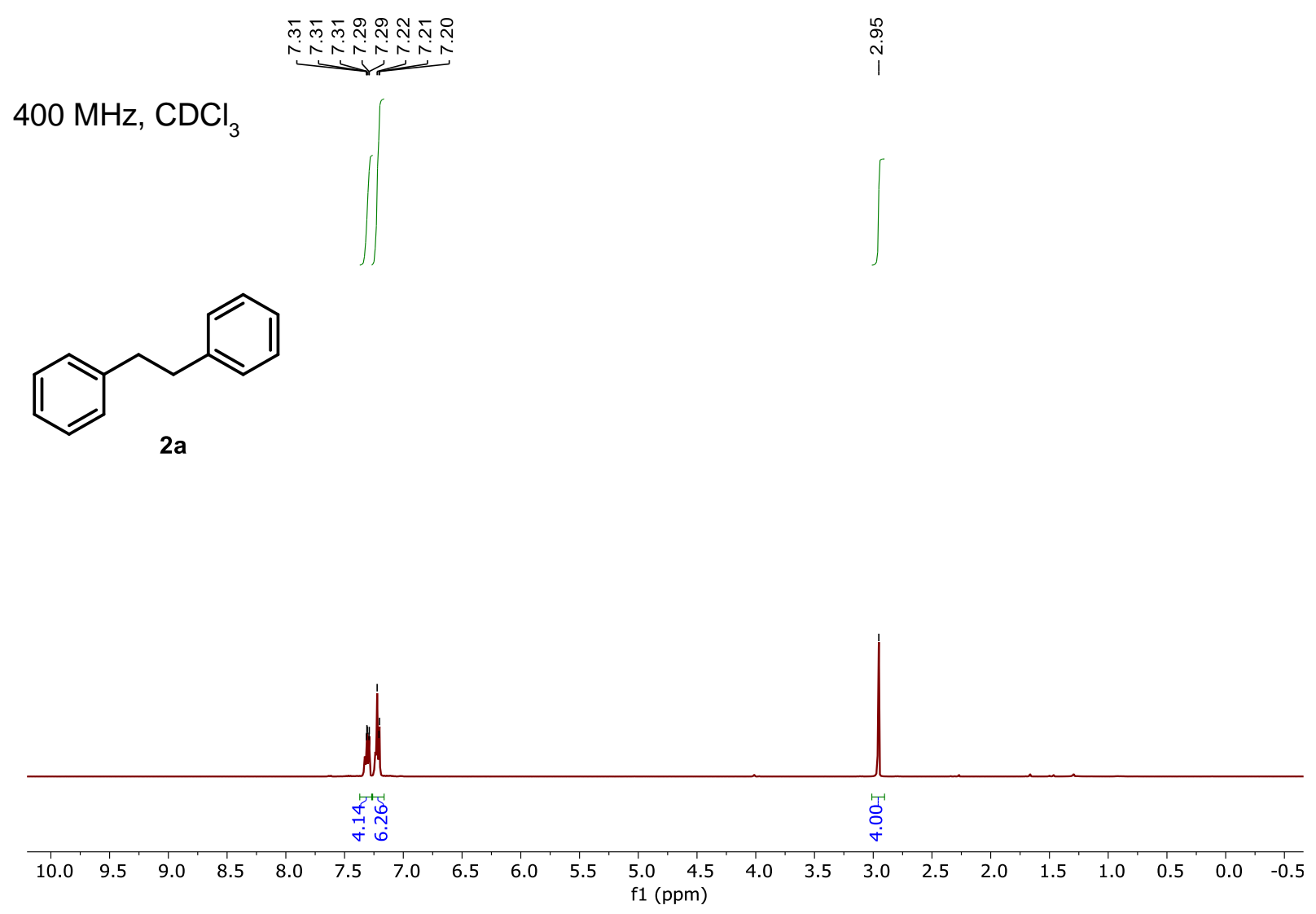

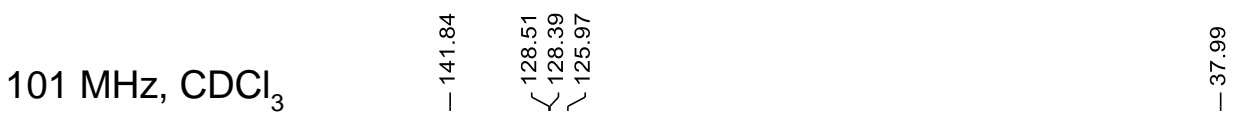<smiles>c1ccc(CCc2ccccc2)cc1</smiles>

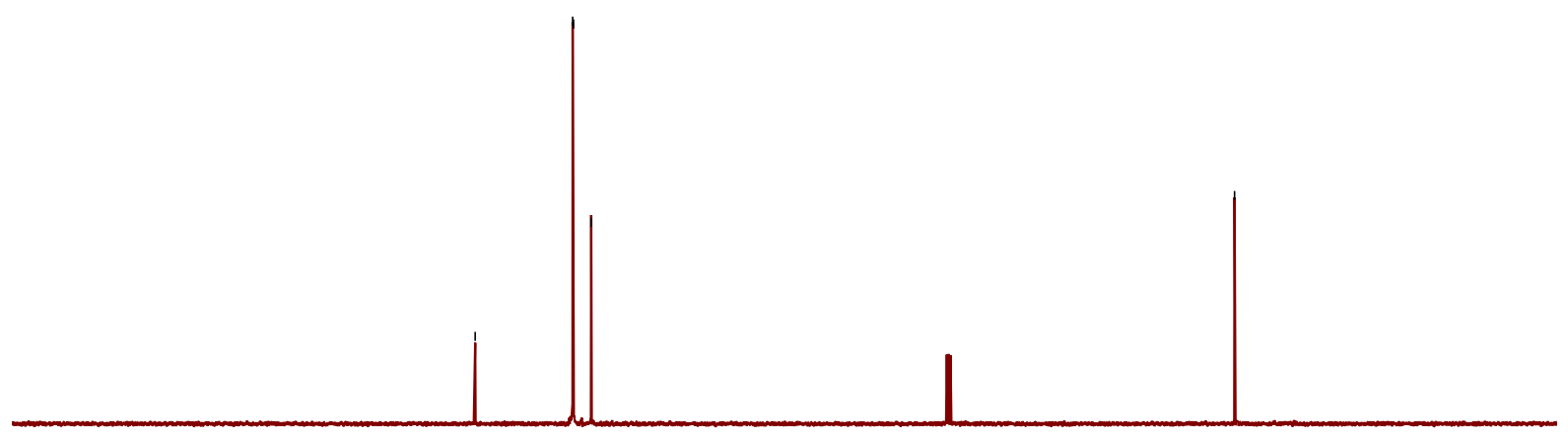

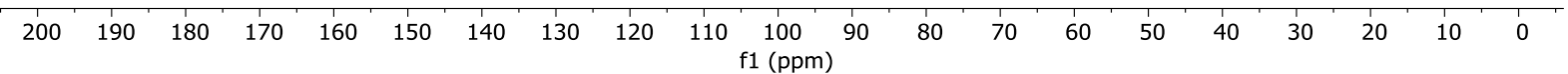




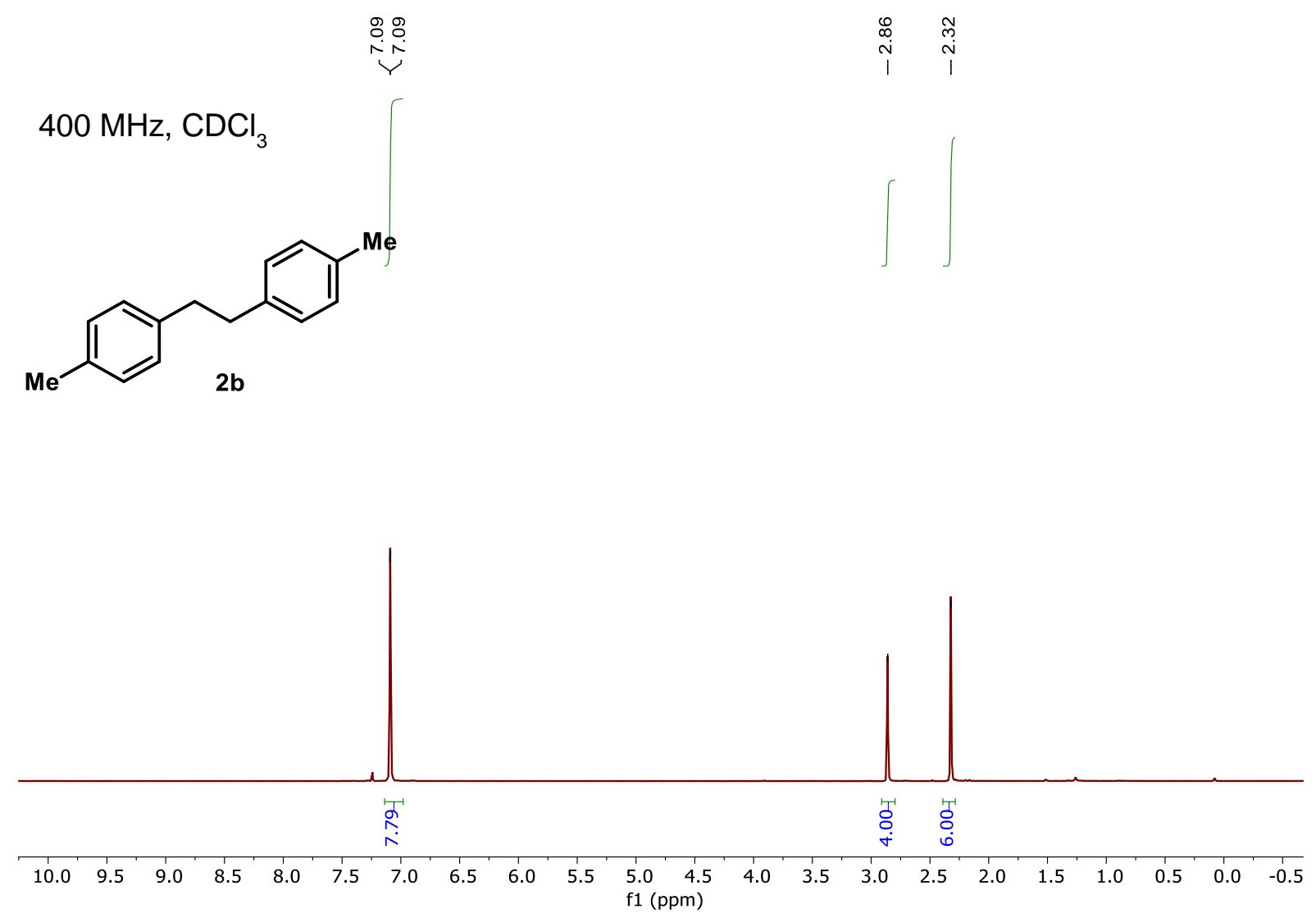

$101 \mathrm{MHz}, \mathrm{CDCl}_{3}$<smiles>Cc1ccc(CCc2ccc(C)cc2)cc1</smiles>

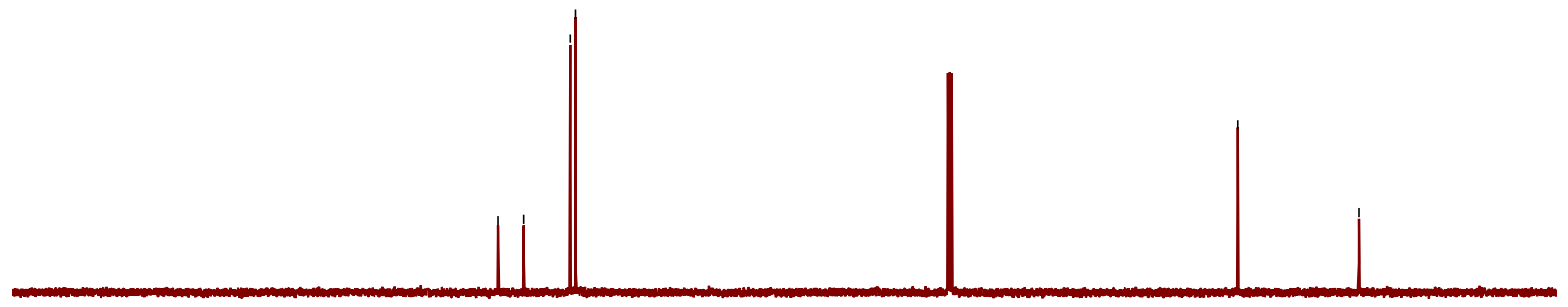

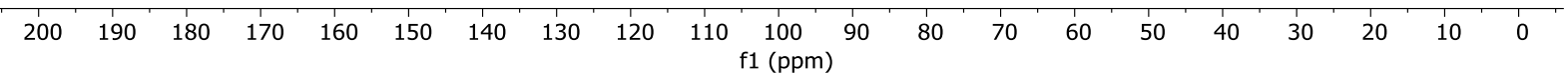



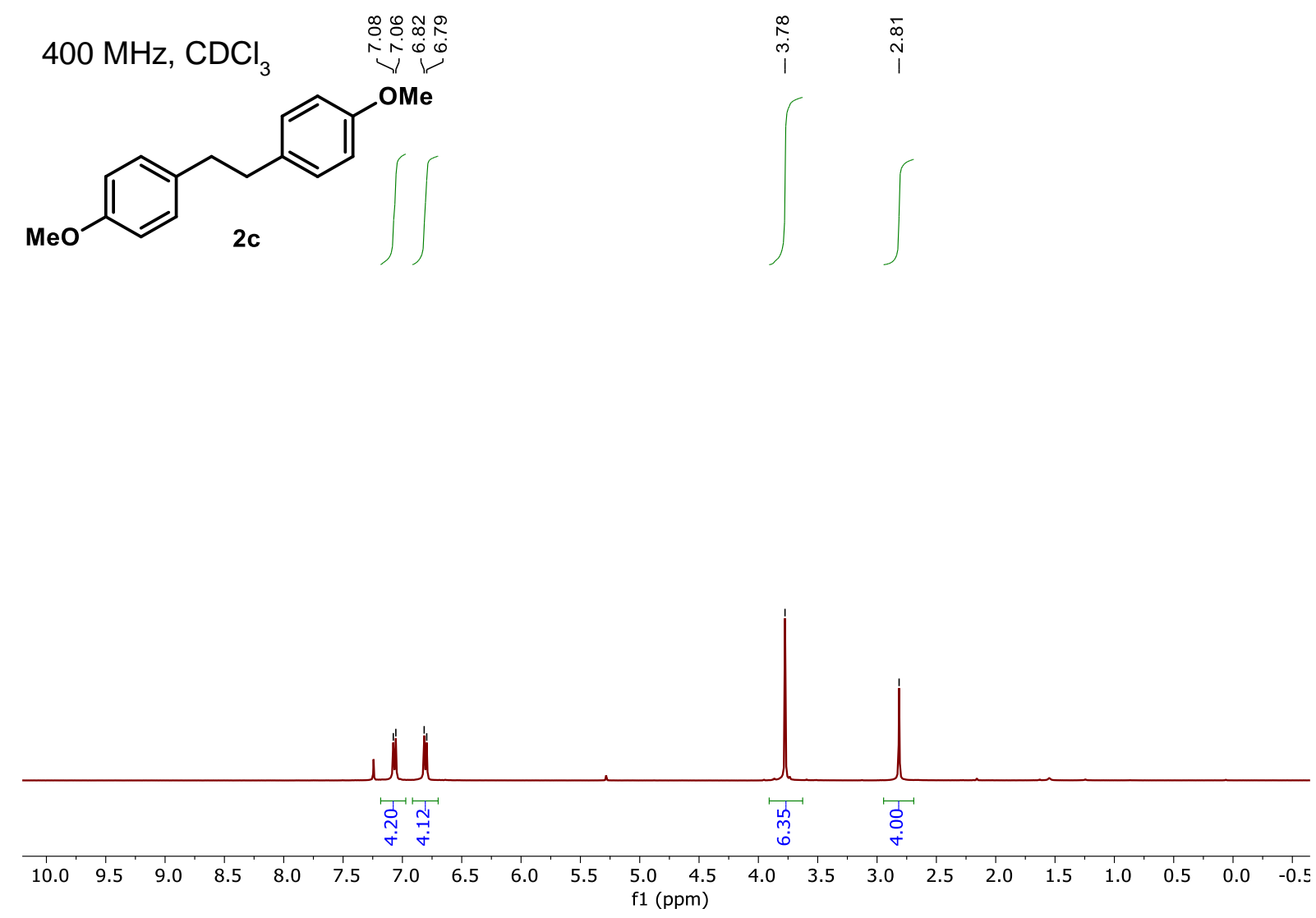

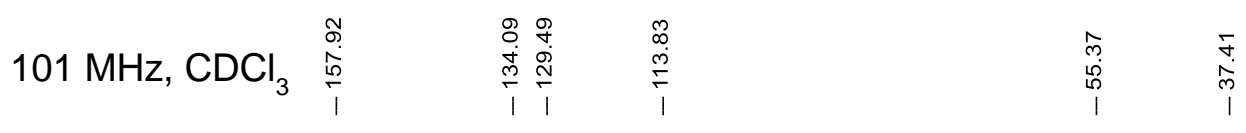<smiles>COc1ccc(CCc2ccc(OC)cc2)cc1</smiles>

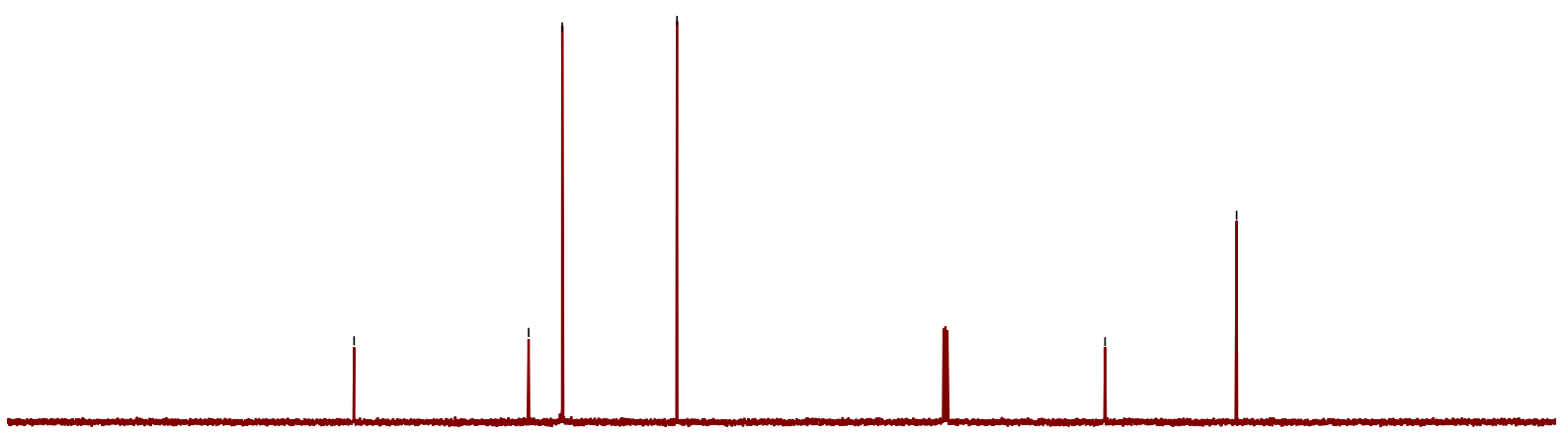

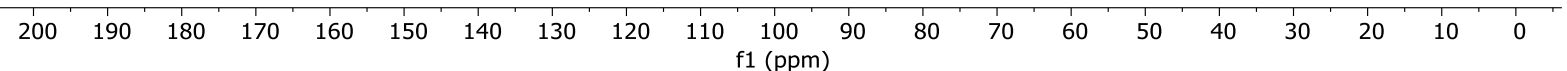




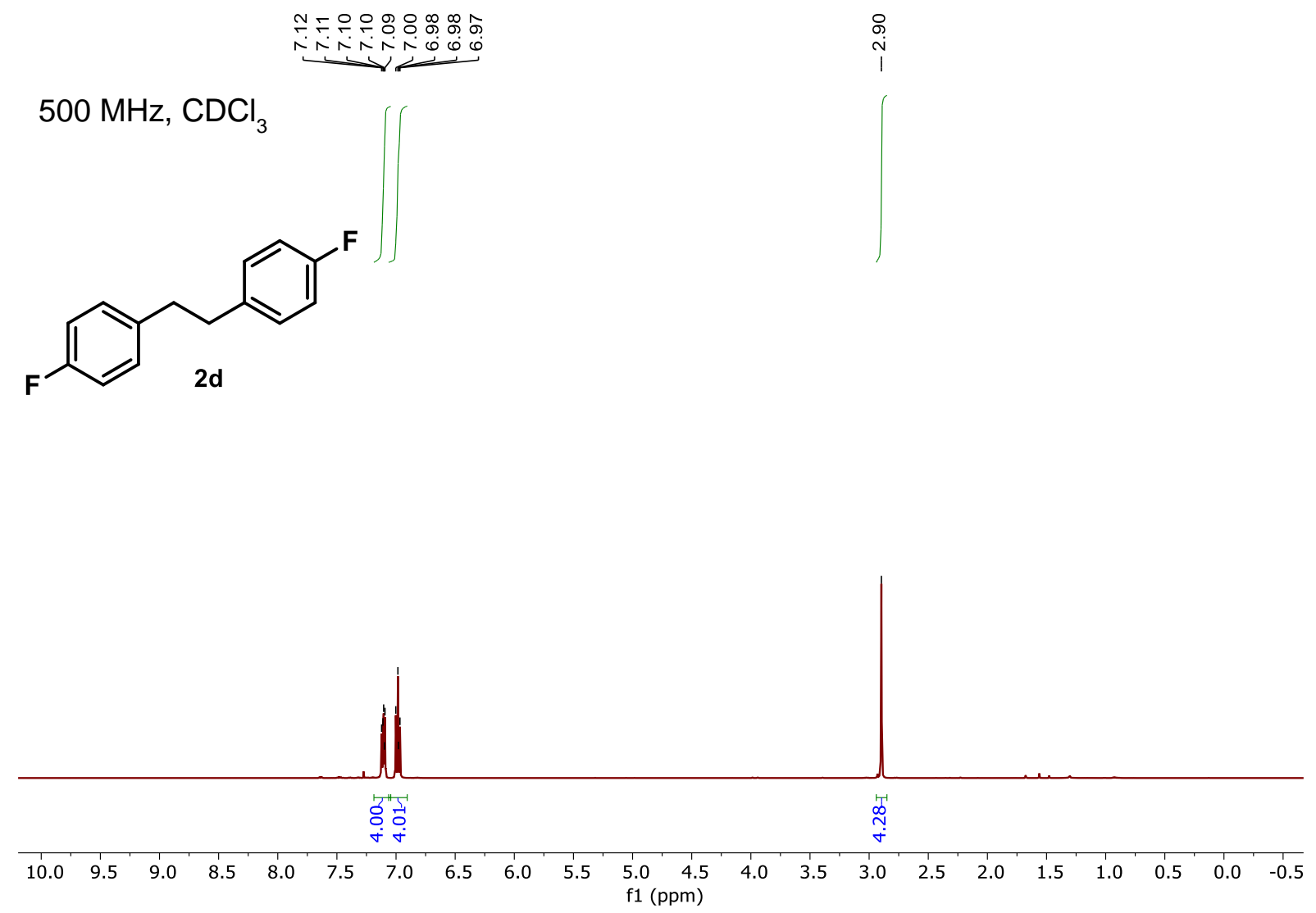

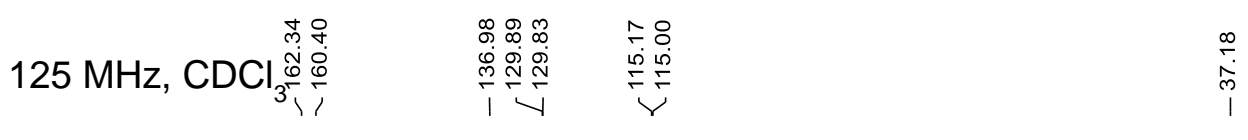<smiles>Fc1ccc(CCc2ccc(F)cc2)cc1</smiles>

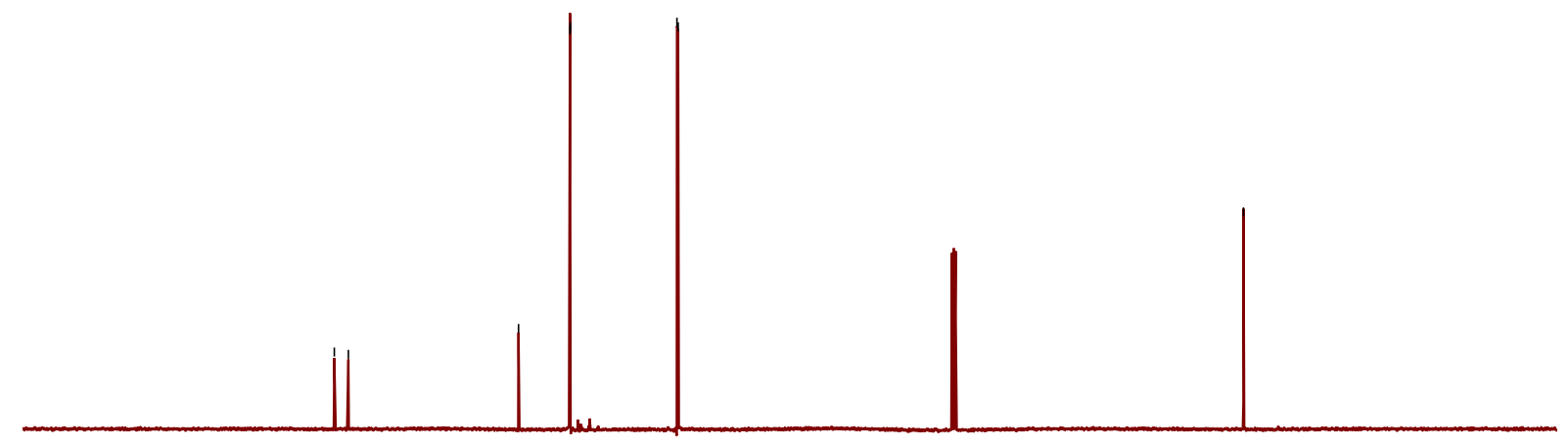

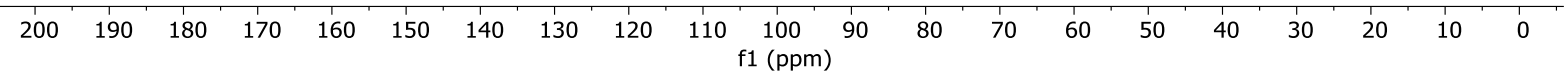




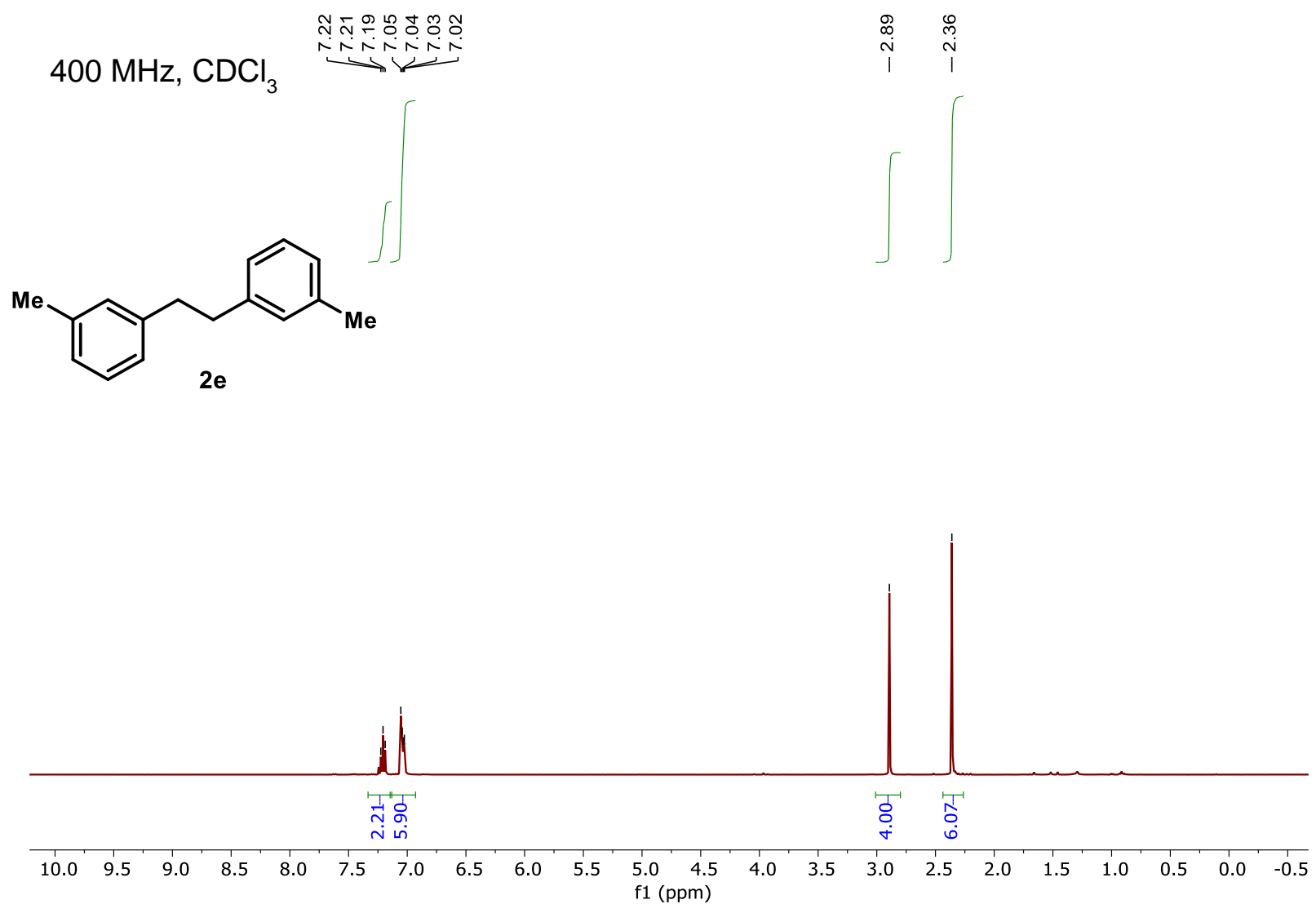

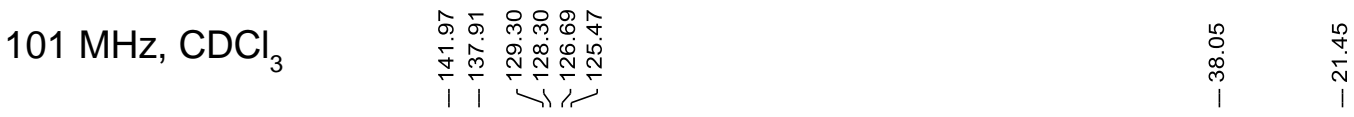<smiles>Cc1cccc(CCc2cccc(C)c2)c1</smiles>

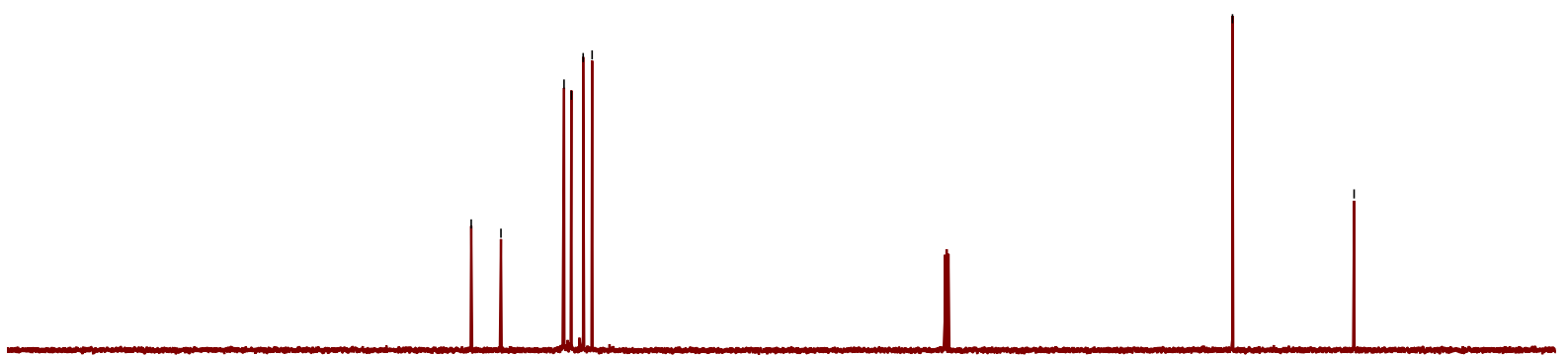

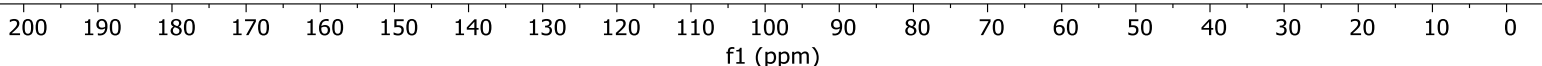




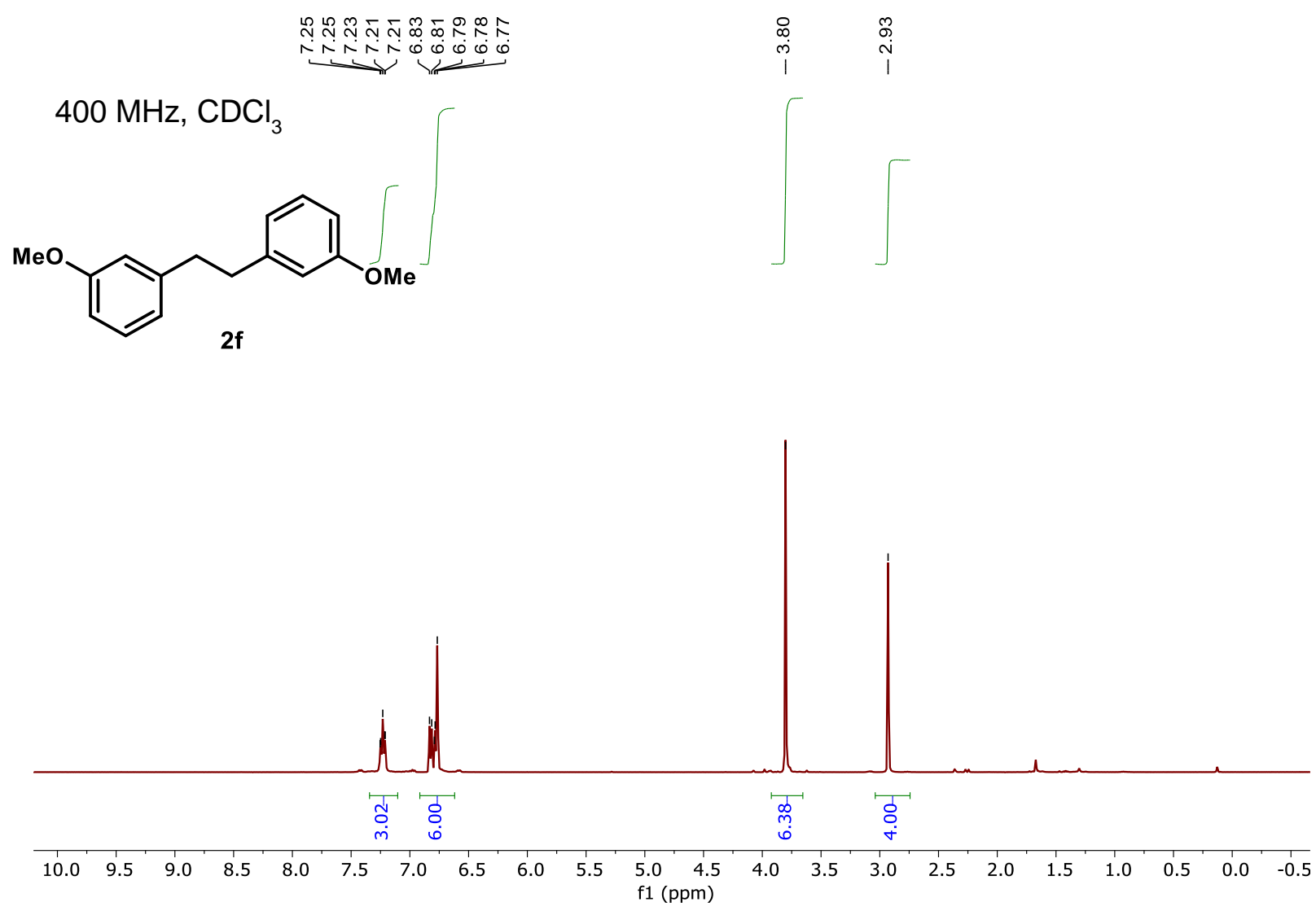

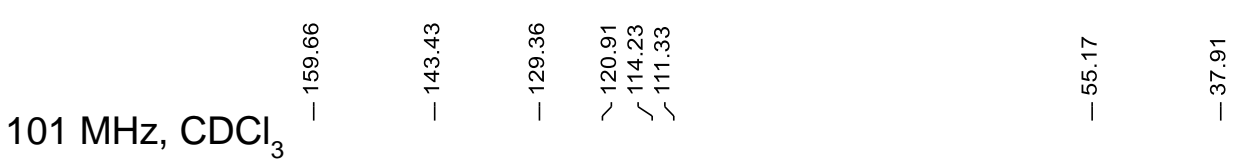<smiles>CCCc1cccc(CCc2cccc(OC)c2)c1</smiles>

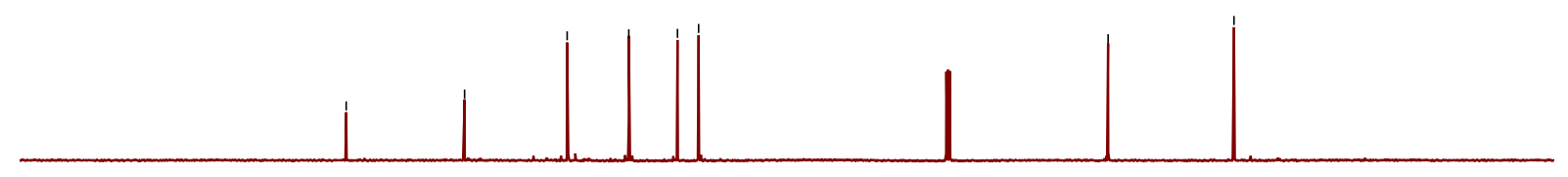

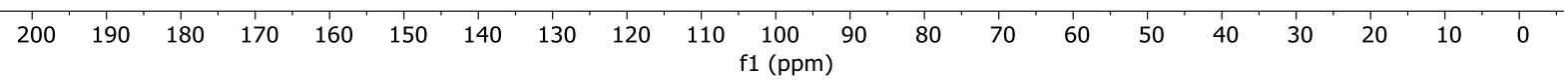



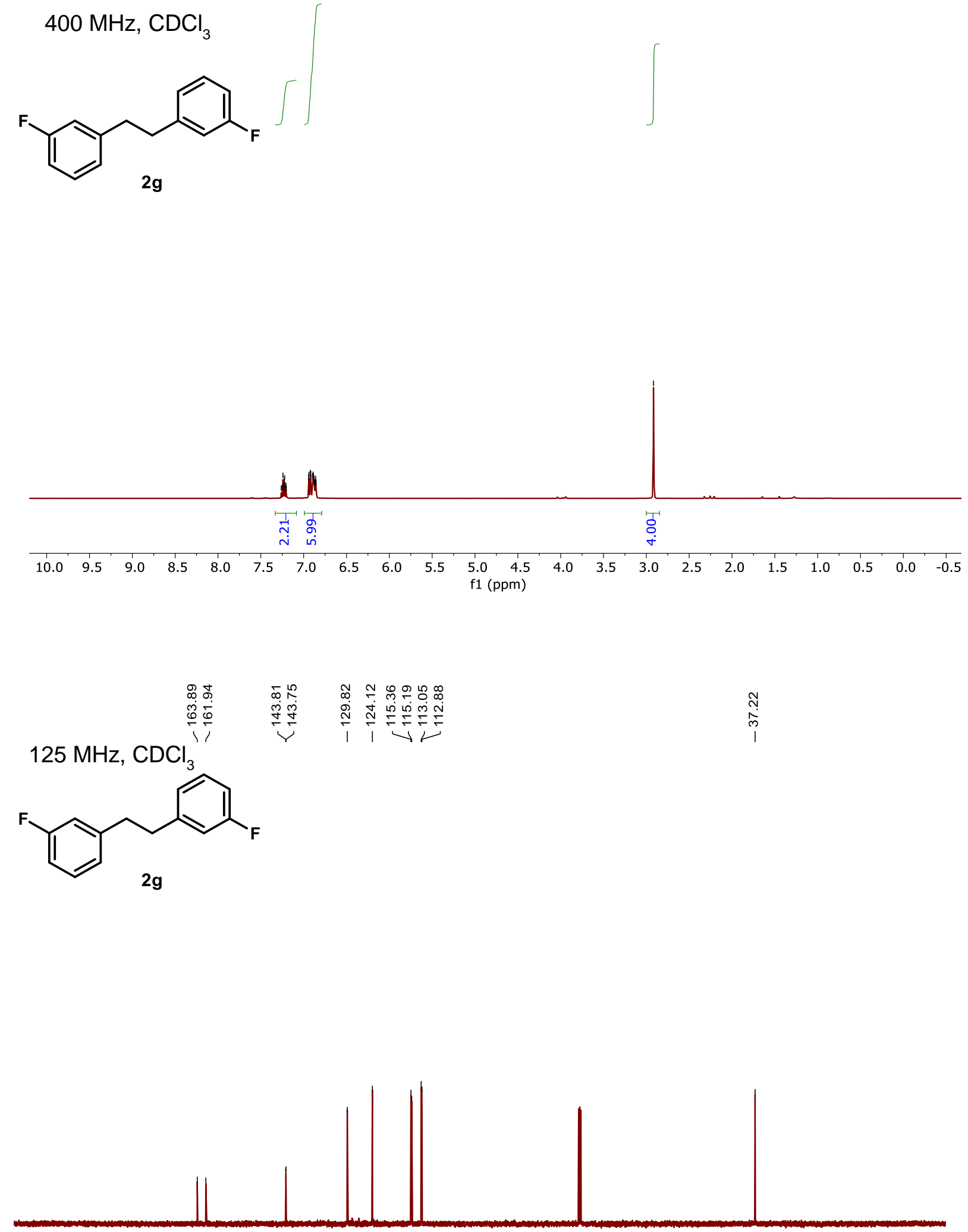

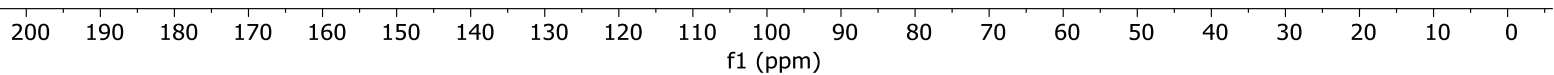




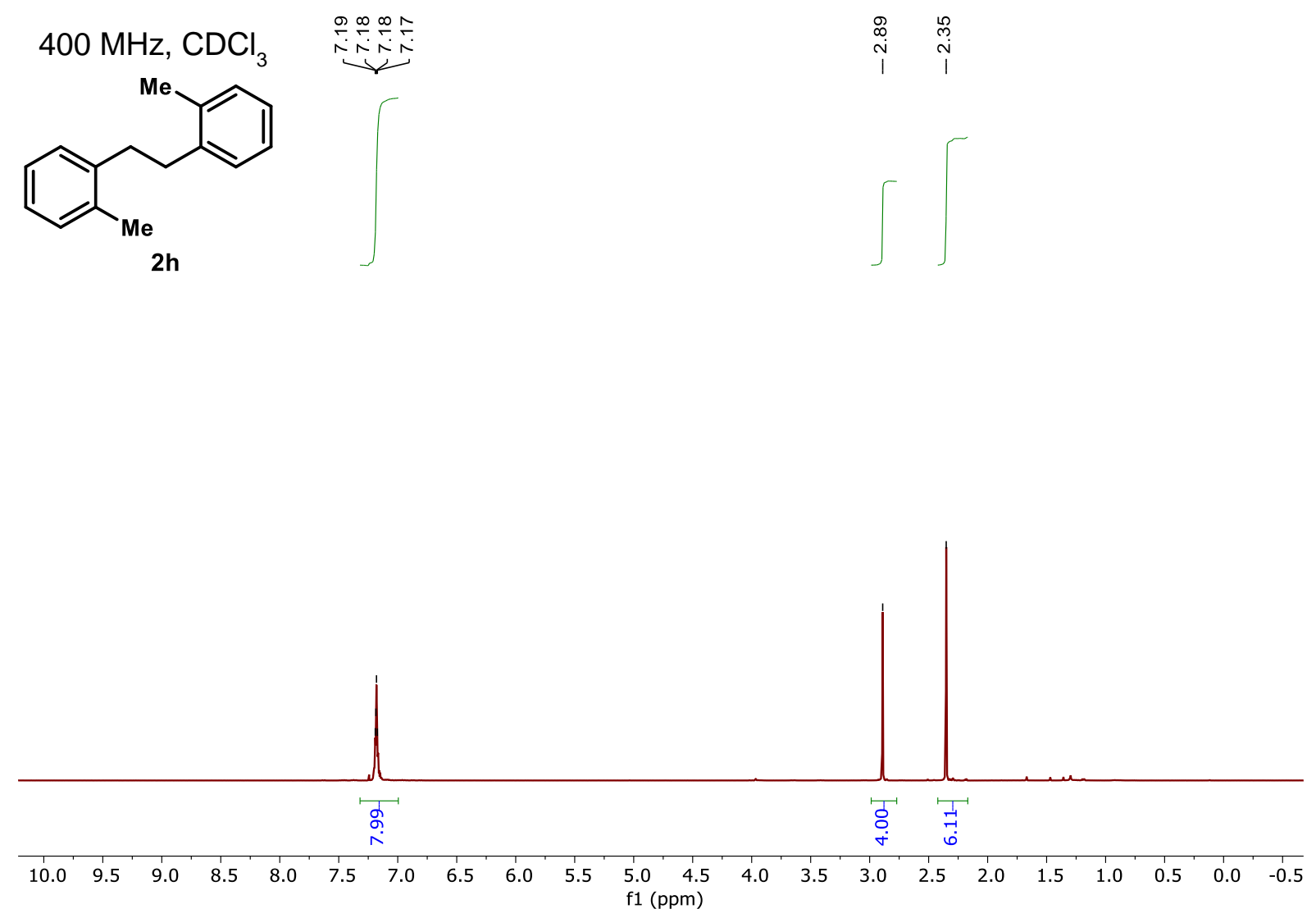

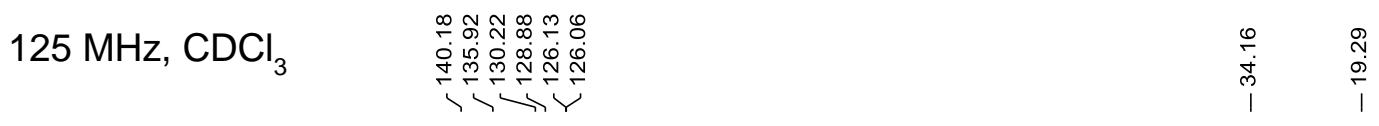<smiles>Cc1ccccc1CCc1ccccc1C</smiles>

2h

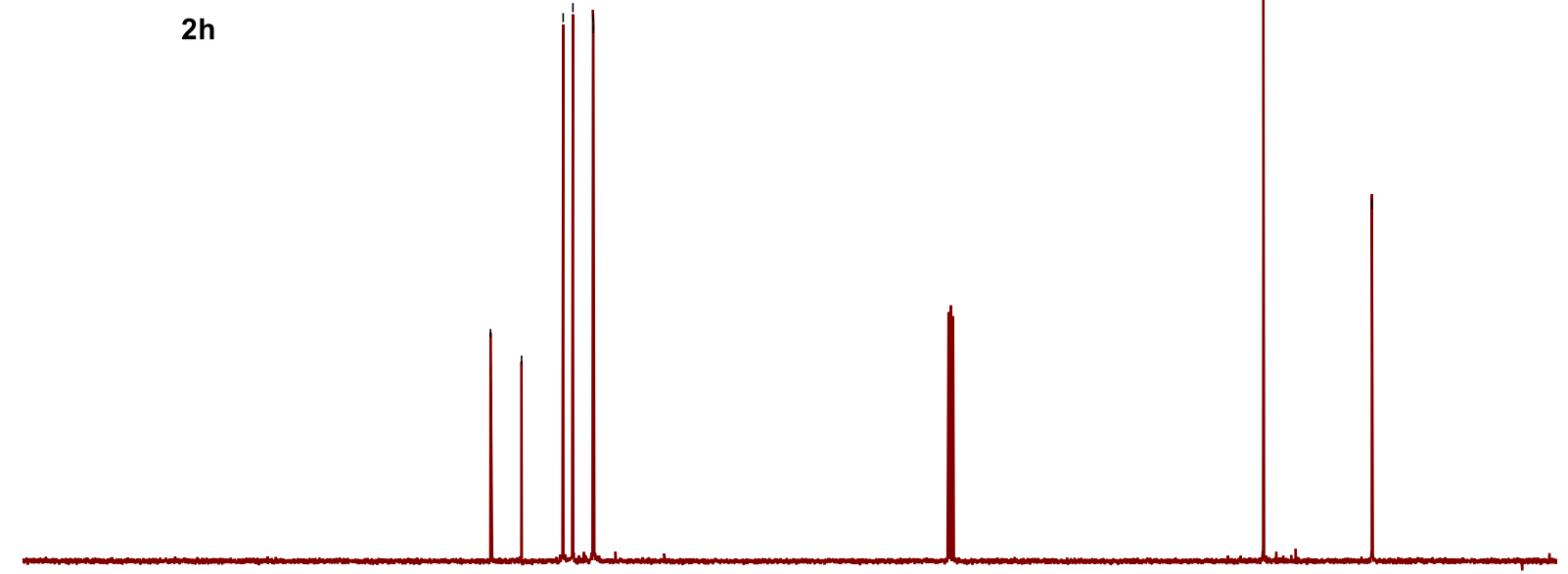

$\begin{array}{lllllllllllllllllllll}200 & 190 & 180 & 170 & 160 & 150 & 140 & 130 & 120 & 110 & \begin{array}{c}100 \\ \mathrm{f} 1(\mathrm{ppm})\end{array} & 90 & 80 & 70 & 60 & 50 & 40 & 30 & 20 & 10 & 0\end{array}$ 

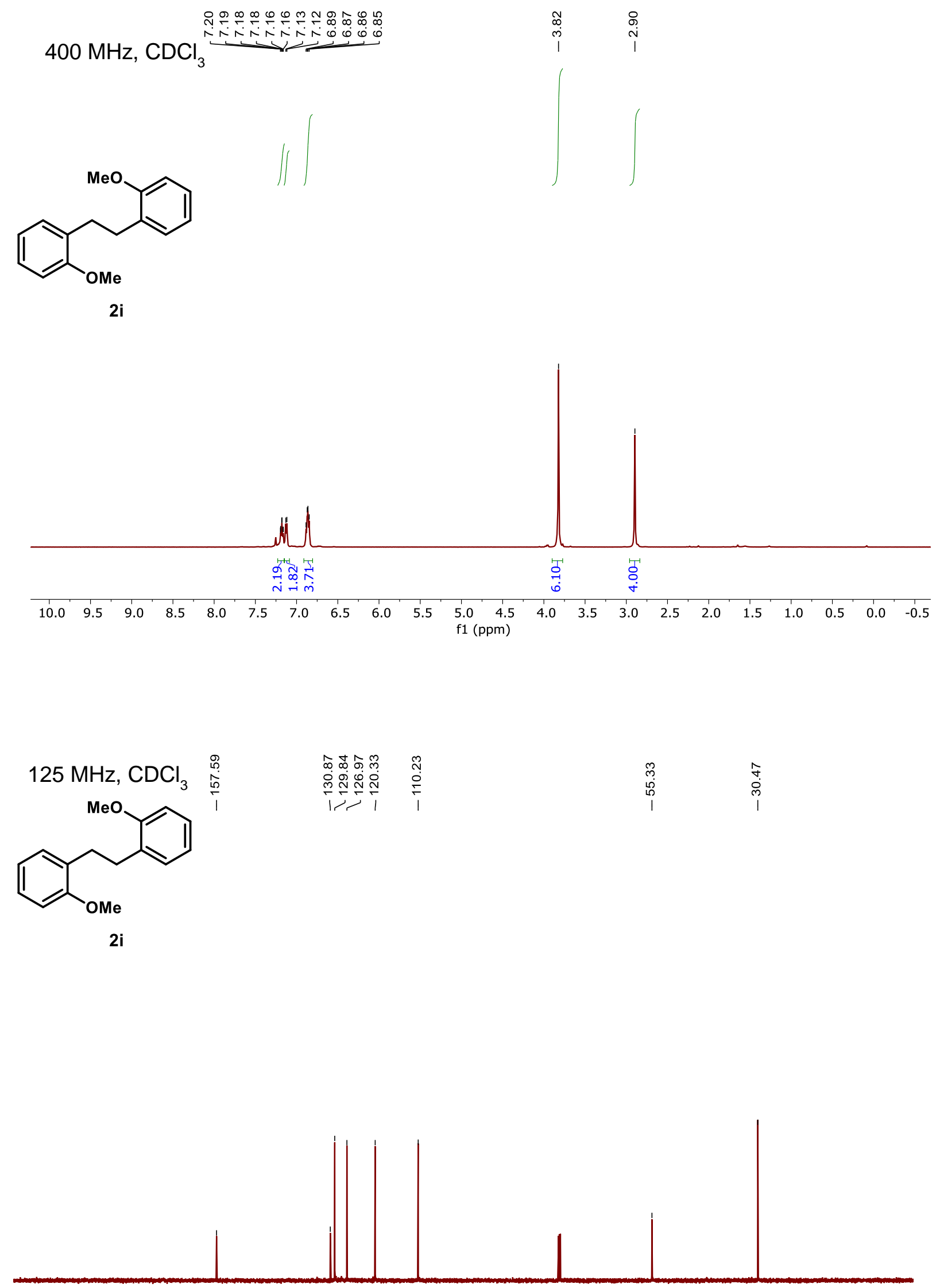

\begin{tabular}{lllllllllllllllllllll}
\hline 200 & 190 & 180 & 170 & 160 & 150 & 140 & 130 & 120 & 110 & $\begin{array}{c}100 \\
\mathrm{f} 1(\mathrm{ppm})\end{array}$ & 90 & 80 & 70 & 60 & 50 & 40 & 30 & 20 & 10 & 0
\end{tabular} 


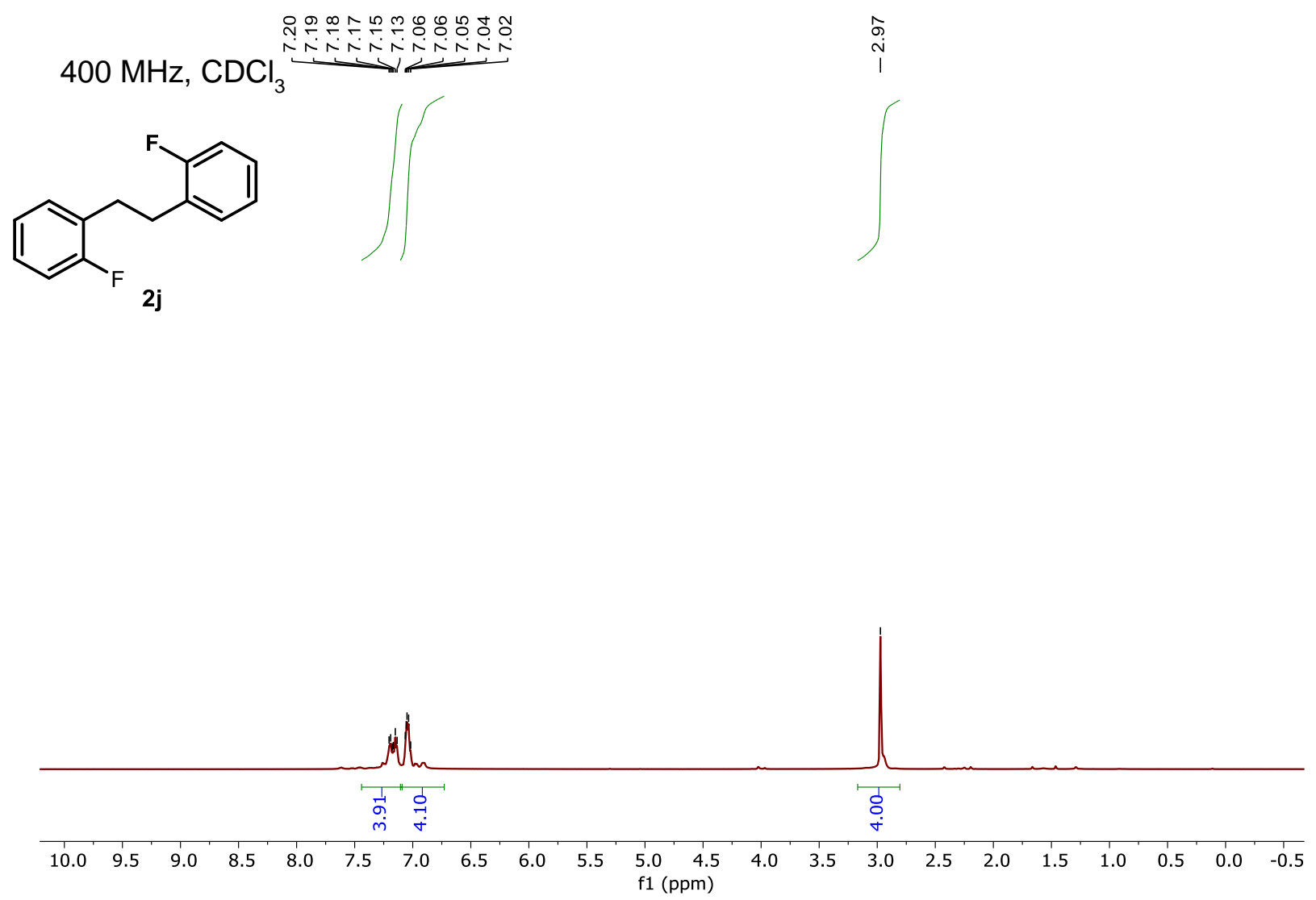<smiles>Fc1ccccc1CCc1ccccc1F</smiles>

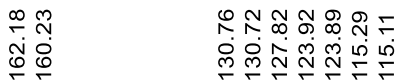

更

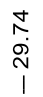

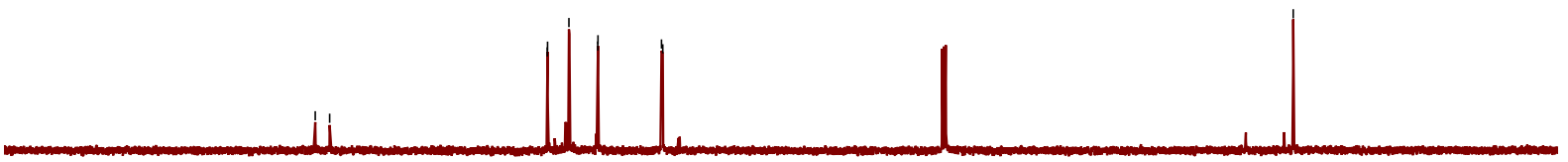




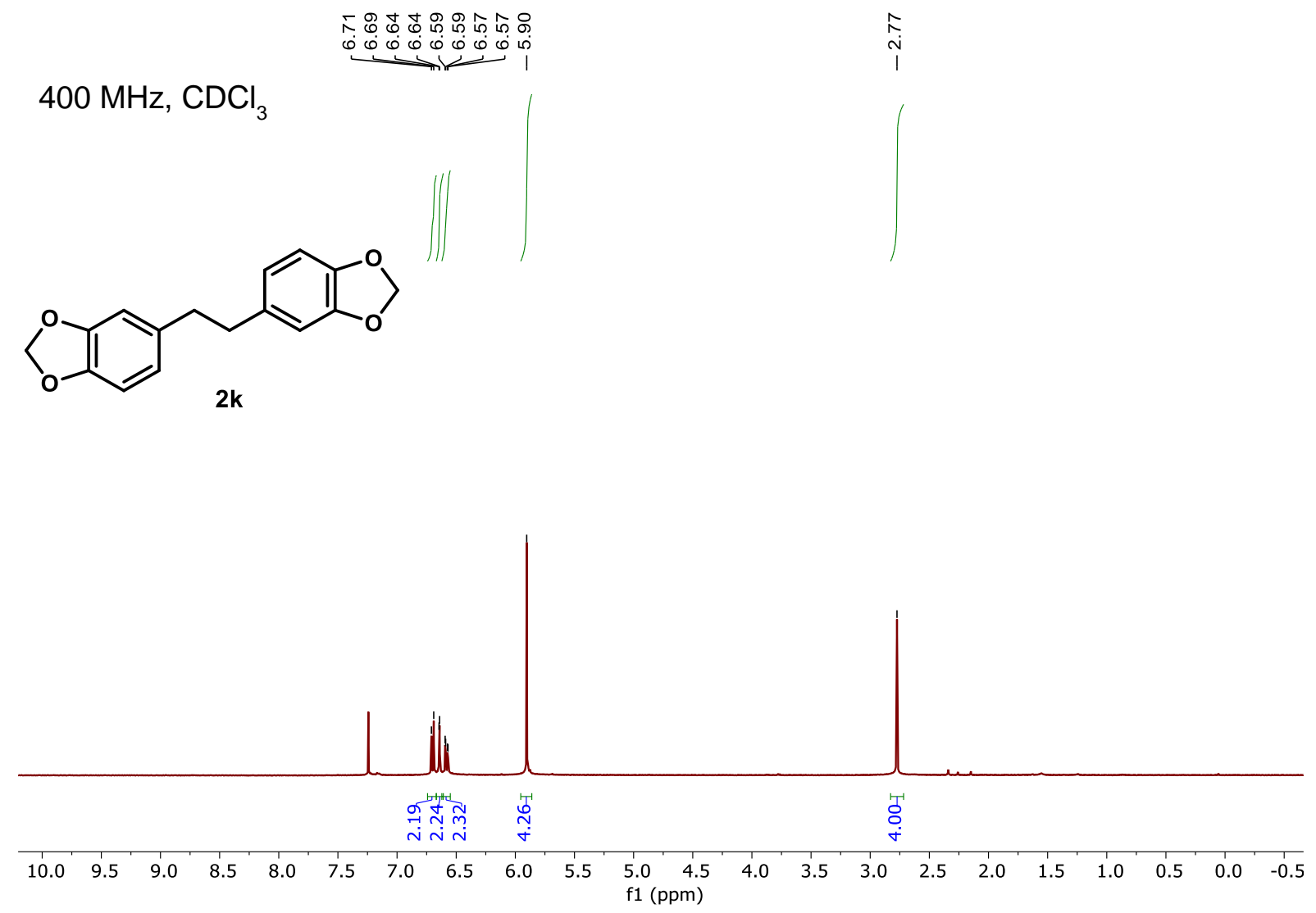

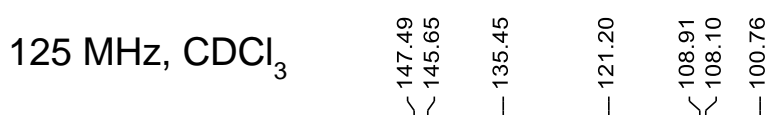<smiles>c1cc2c(cc1CCc1ccc3c(c1)OCO3)OCO2</smiles>

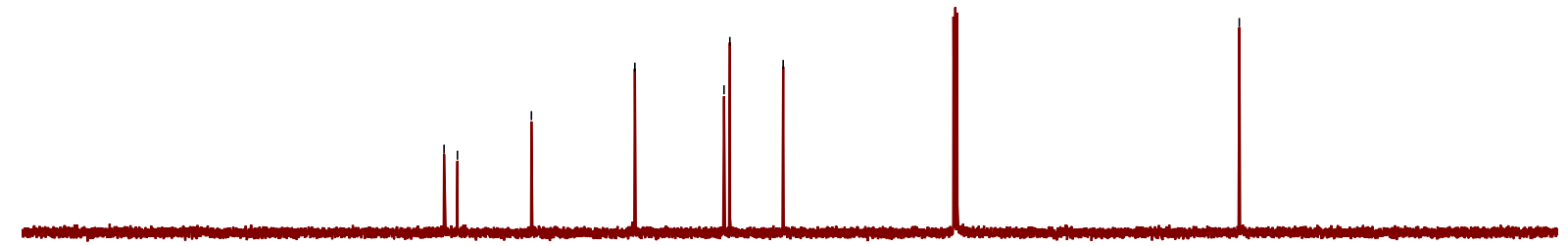

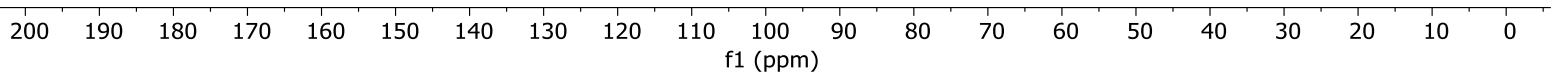




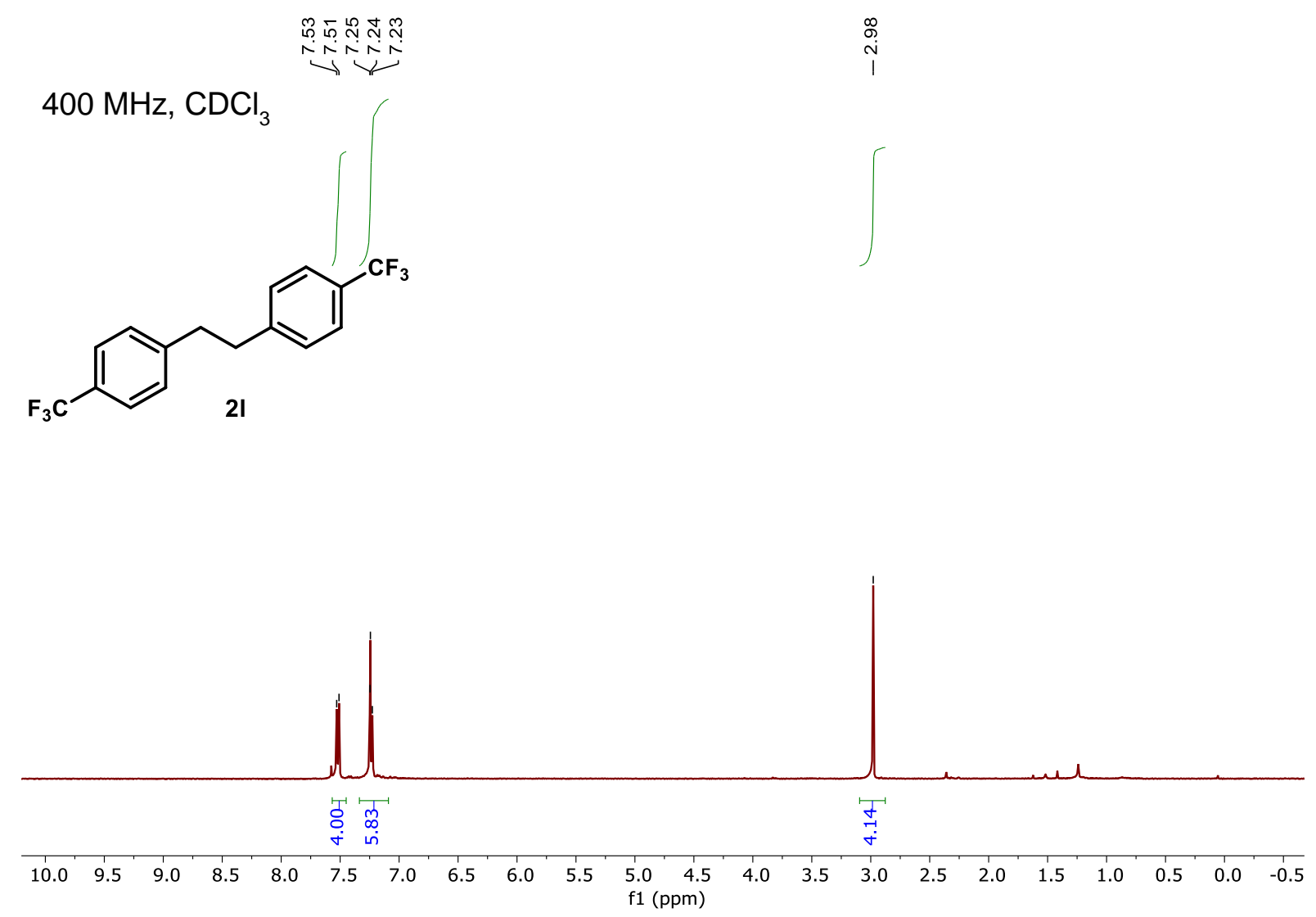

$101 \mathrm{MHz}, \mathrm{CDCl}_{3}$ 实

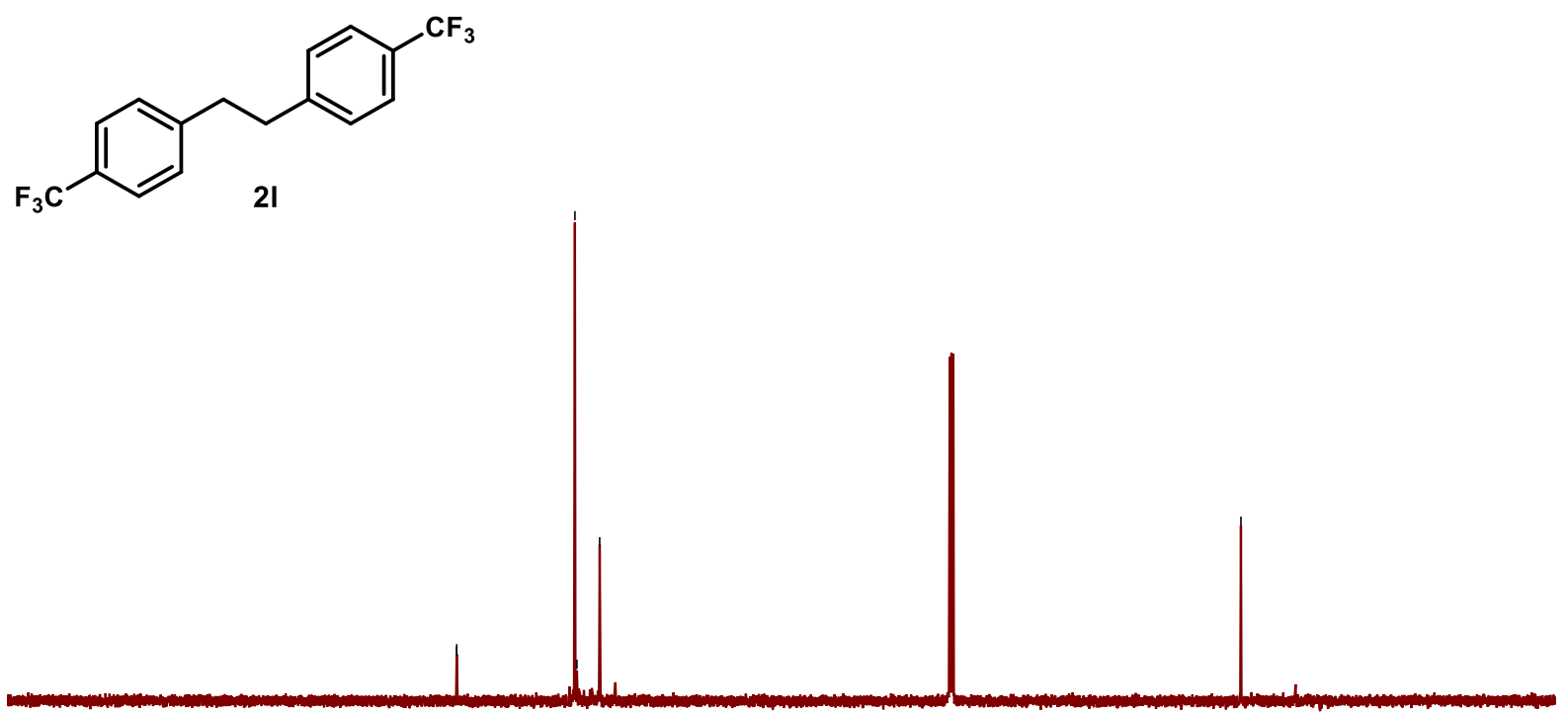

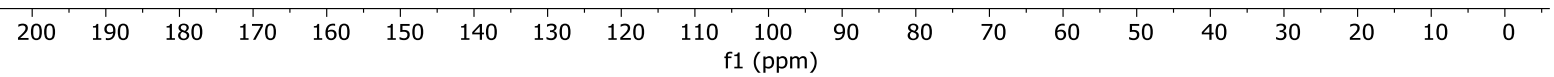




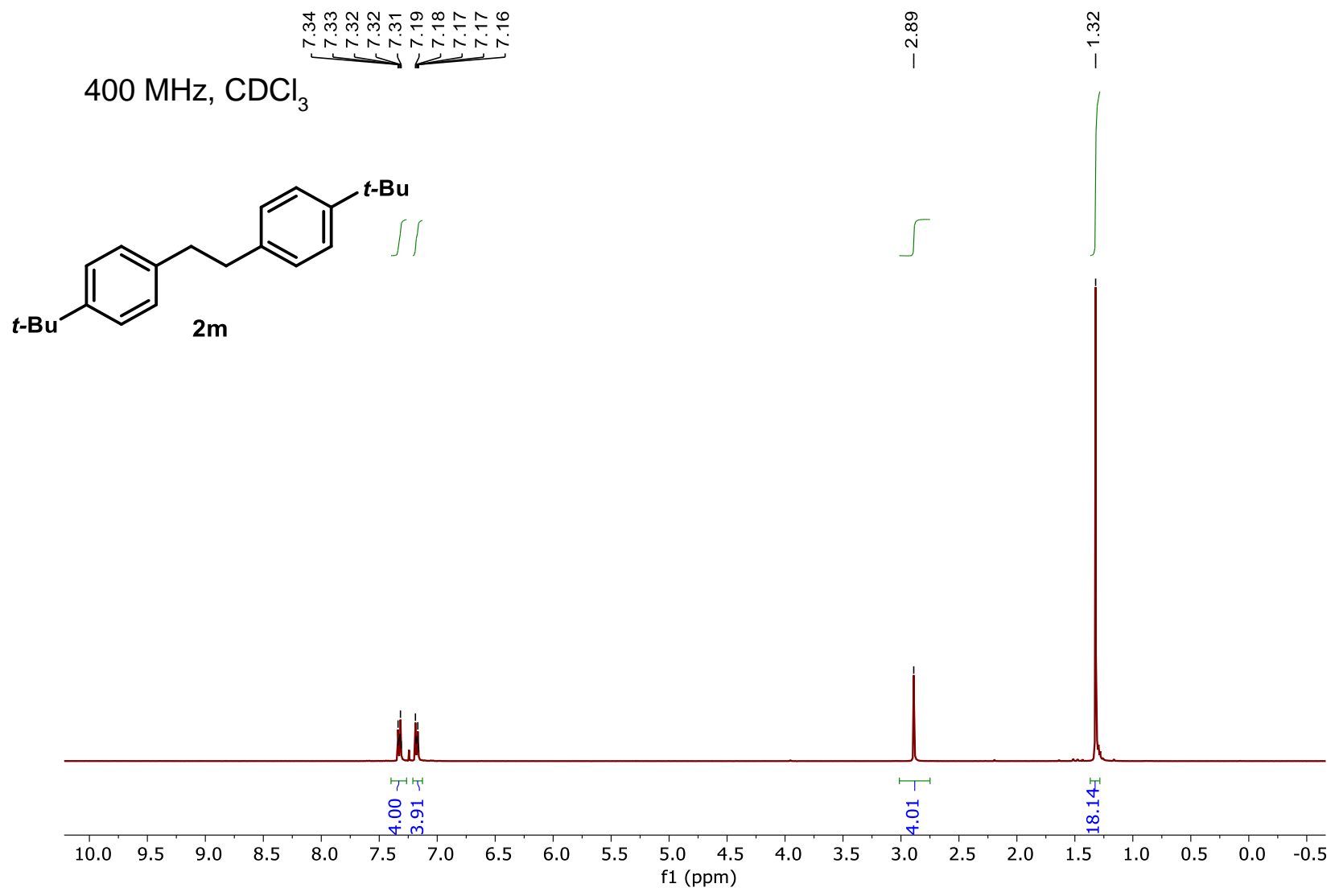

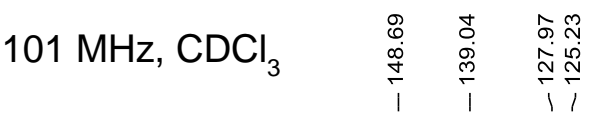

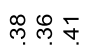

लिं

$\overbrace{2 \mathrm{C}}^{t-\mathrm{Bu}}$

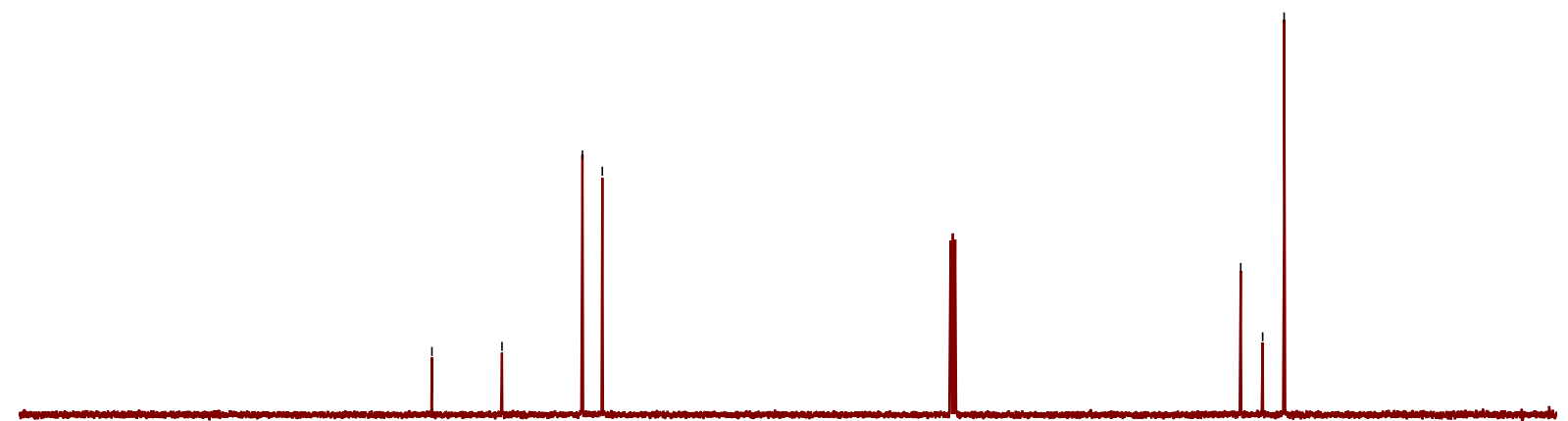

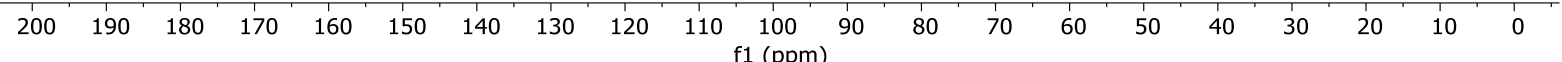




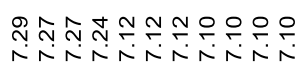

$400 \mathrm{MHz} \mathrm{CDCl}_{3}$<smiles>CC(C)(C)c1ccc([N+](=O)[O-])cc1</smiles>

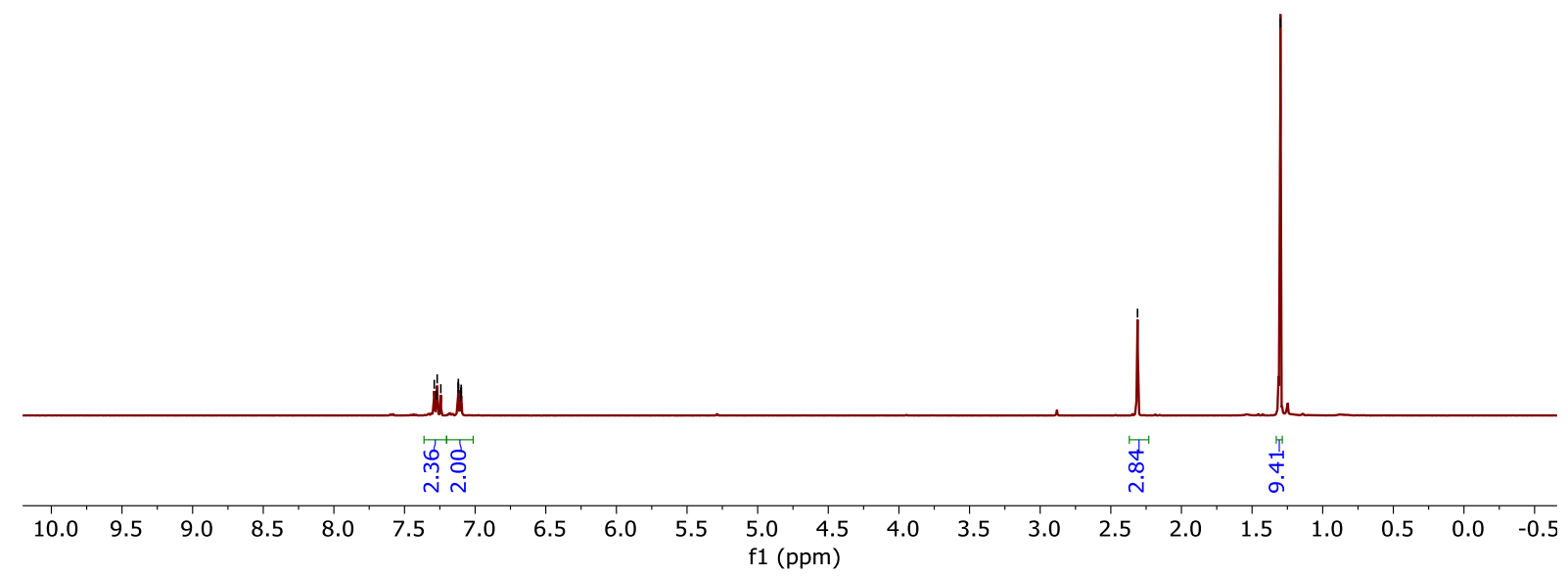


$400 \mathrm{MHz}, \mathrm{CDCl}_{3}$

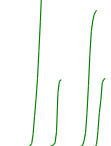<smiles>c1ccc2cc(CCc3ccc4ccccc4c3)ccc2c1</smiles>

2n

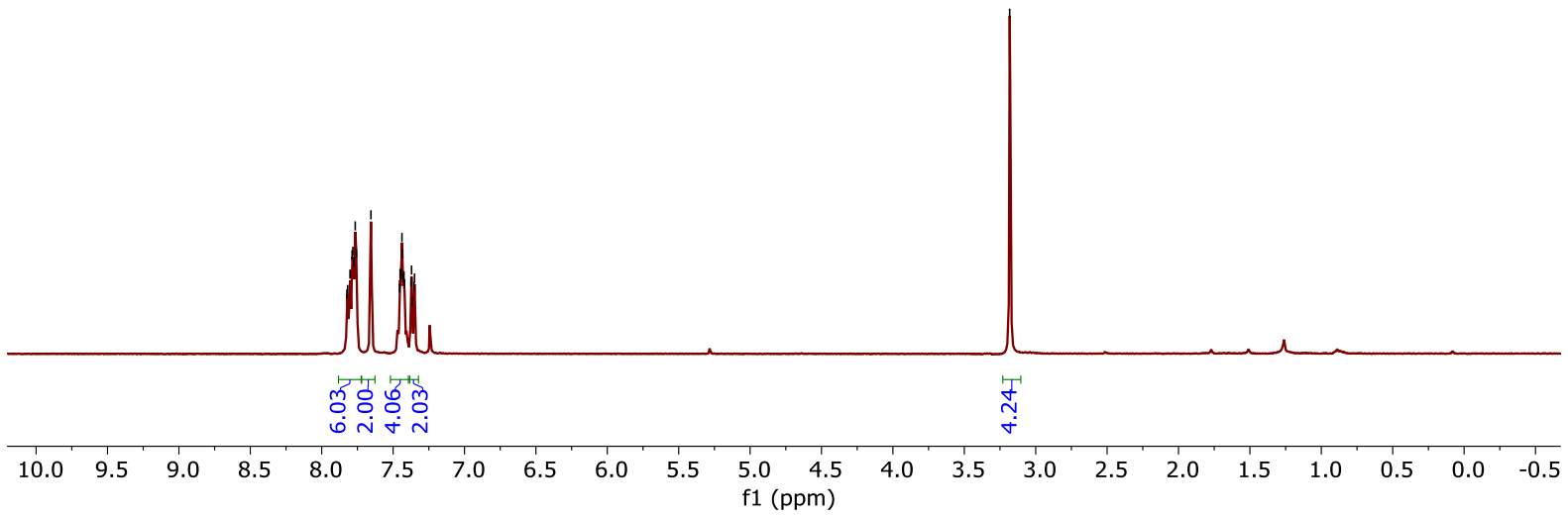

$125 \mathrm{MHz}, \mathrm{CDCl}_{3}$<smiles>c1ccc2cc(CCc3ccc4ccccc4c3)ccc2c1</smiles>

2n

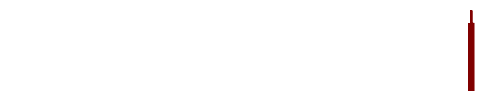

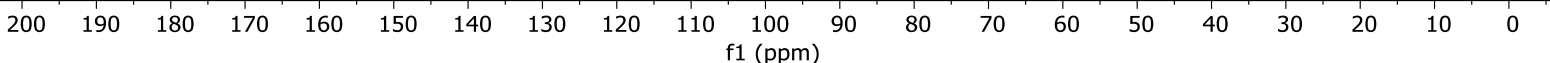




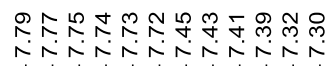

$400 \mathrm{MHz}, \mathrm{CDCl}_{3}$<smiles>Cc1ccc2ccccc2c1</smiles>

$4 n$

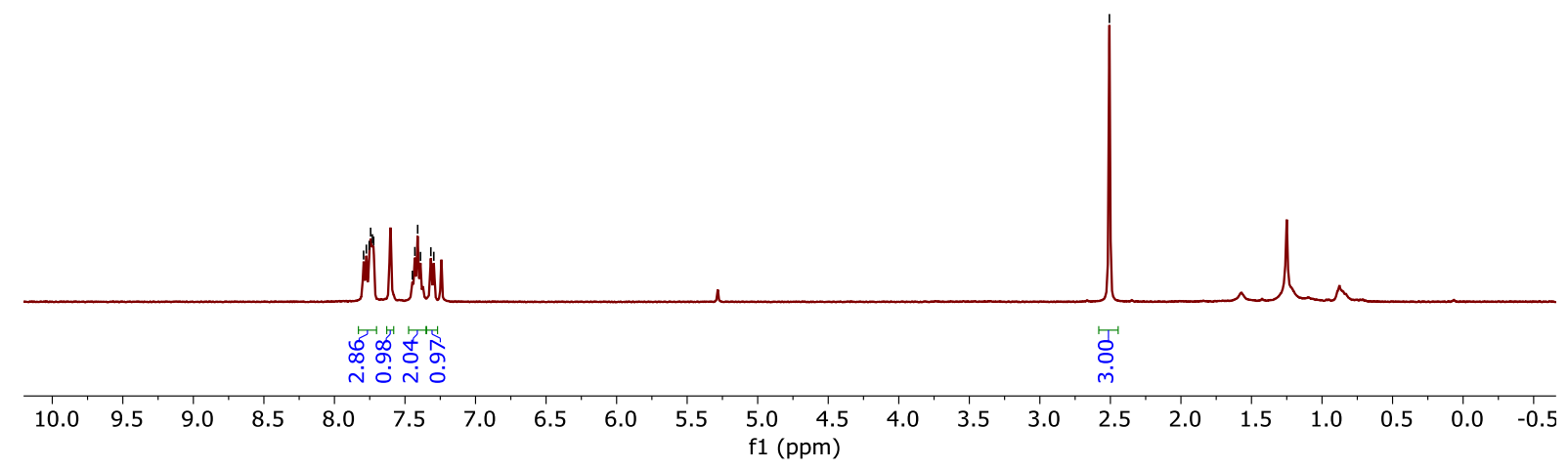



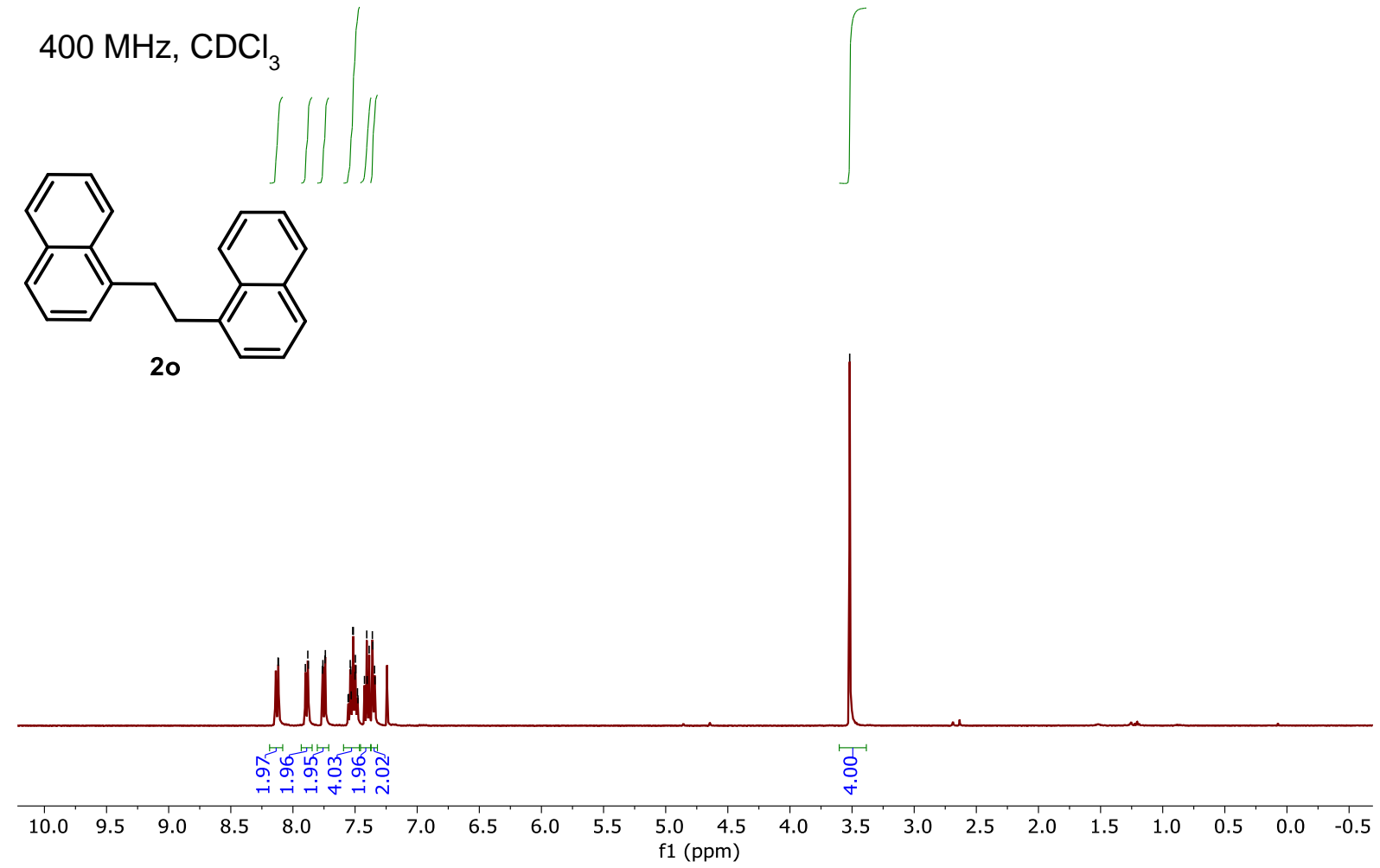

$101 \mathrm{MHz}, \mathrm{CDCl}_{3}$

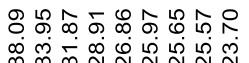

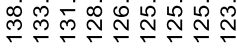<smiles>c1ccc2c(CCc3cccc4ccccc34)cccc2c1</smiles>

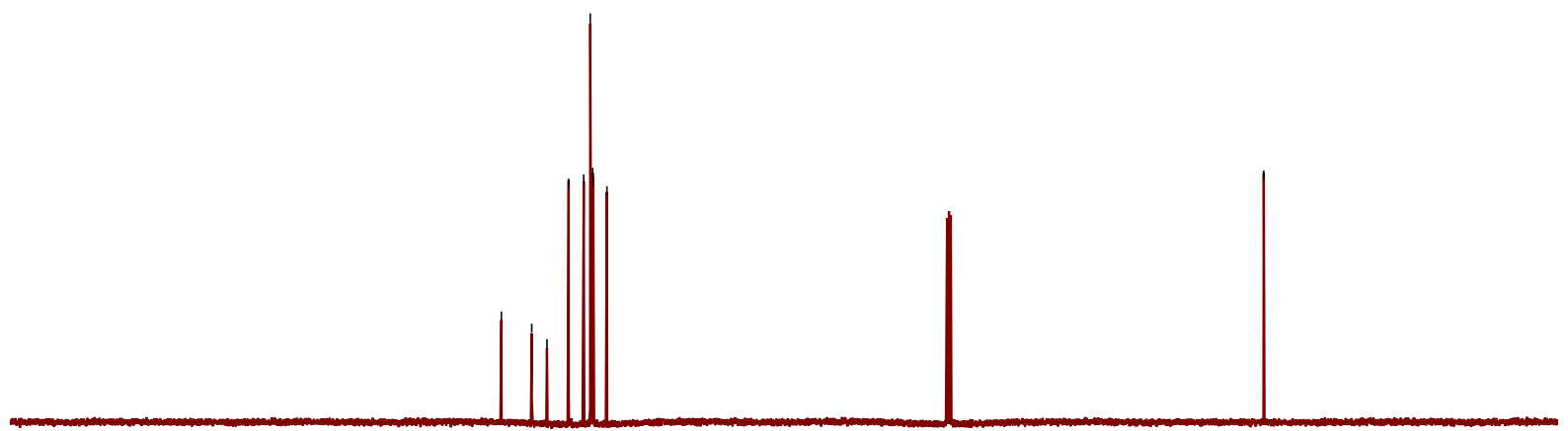

$\begin{array}{llllllllllllllllllllll}200 & 190 & 180 & 170 & 160 & 150 & 140 & 130 & 120 & 110 & 100 & 90 & 80 & 70 & 60 & 50 & 40 & 30 & 20 & 10 & 0\end{array}$ 
$400 \mathrm{MHz}, \mathrm{CDCl}_{3}$<smiles>[X]c1cccc2ccccc12</smiles>

40

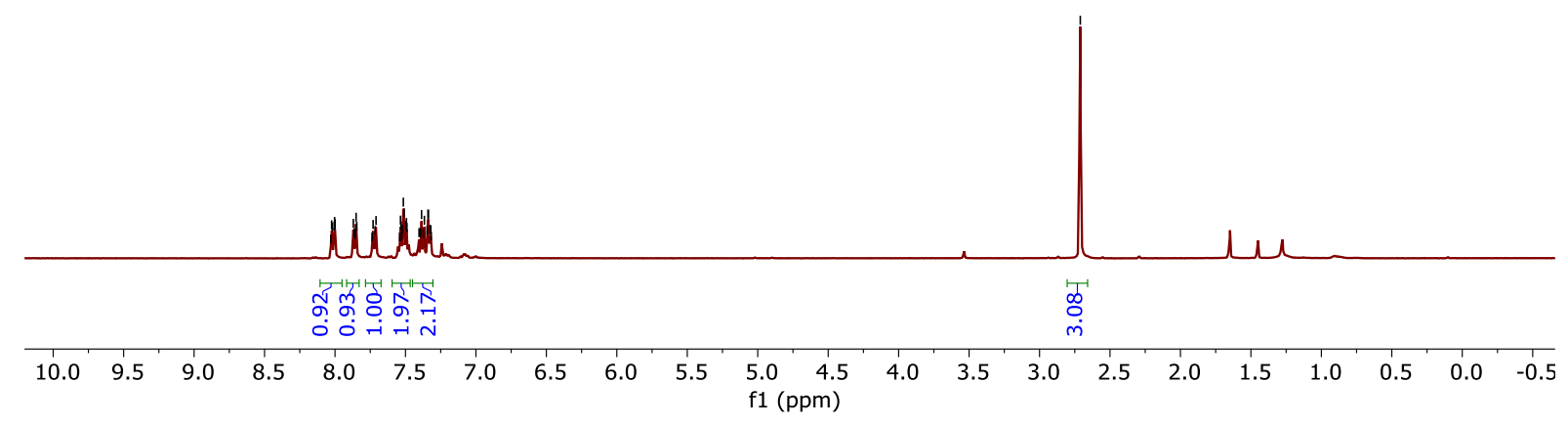


礐 尔

$400 \mathrm{MHz}, \mathrm{CDCl}_{3}$<smiles>CC(=O)c1ccc(CCc2ccc(C(C)=O)cc2)cc1</smiles>

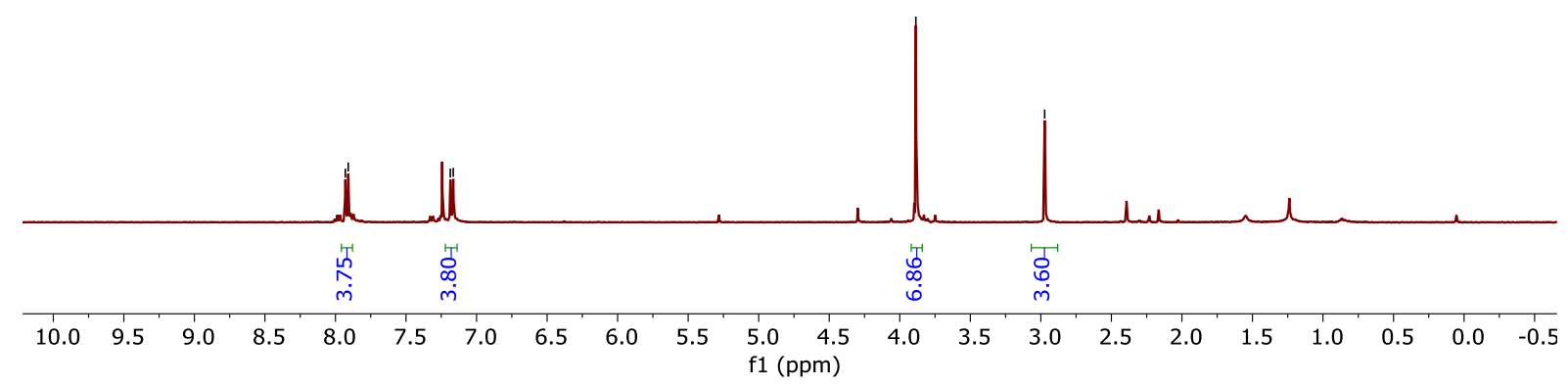




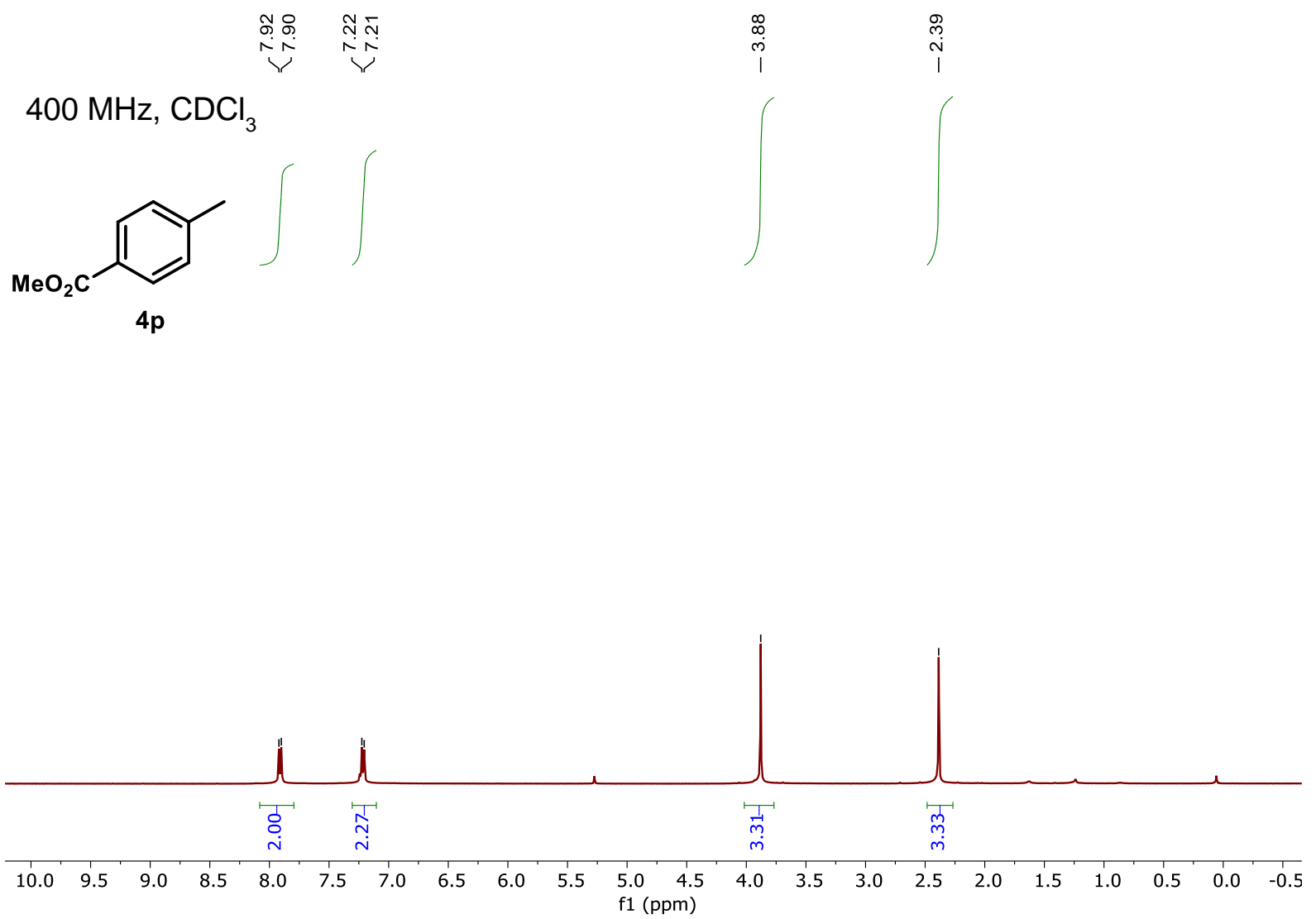

$125 \mathrm{MHz}, \mathrm{CDCl}_{3}$<smiles>CC(=O)c1ccc(C)cc1</smiles>

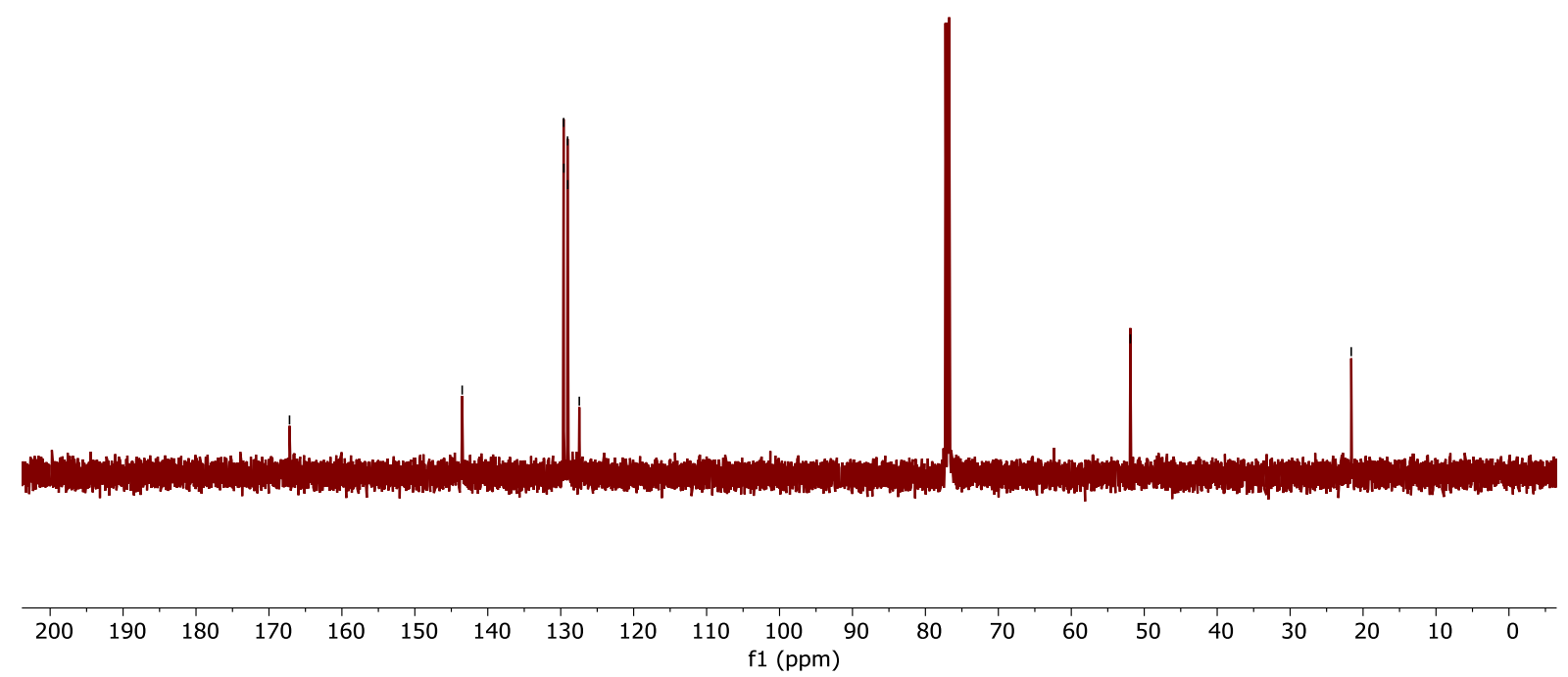




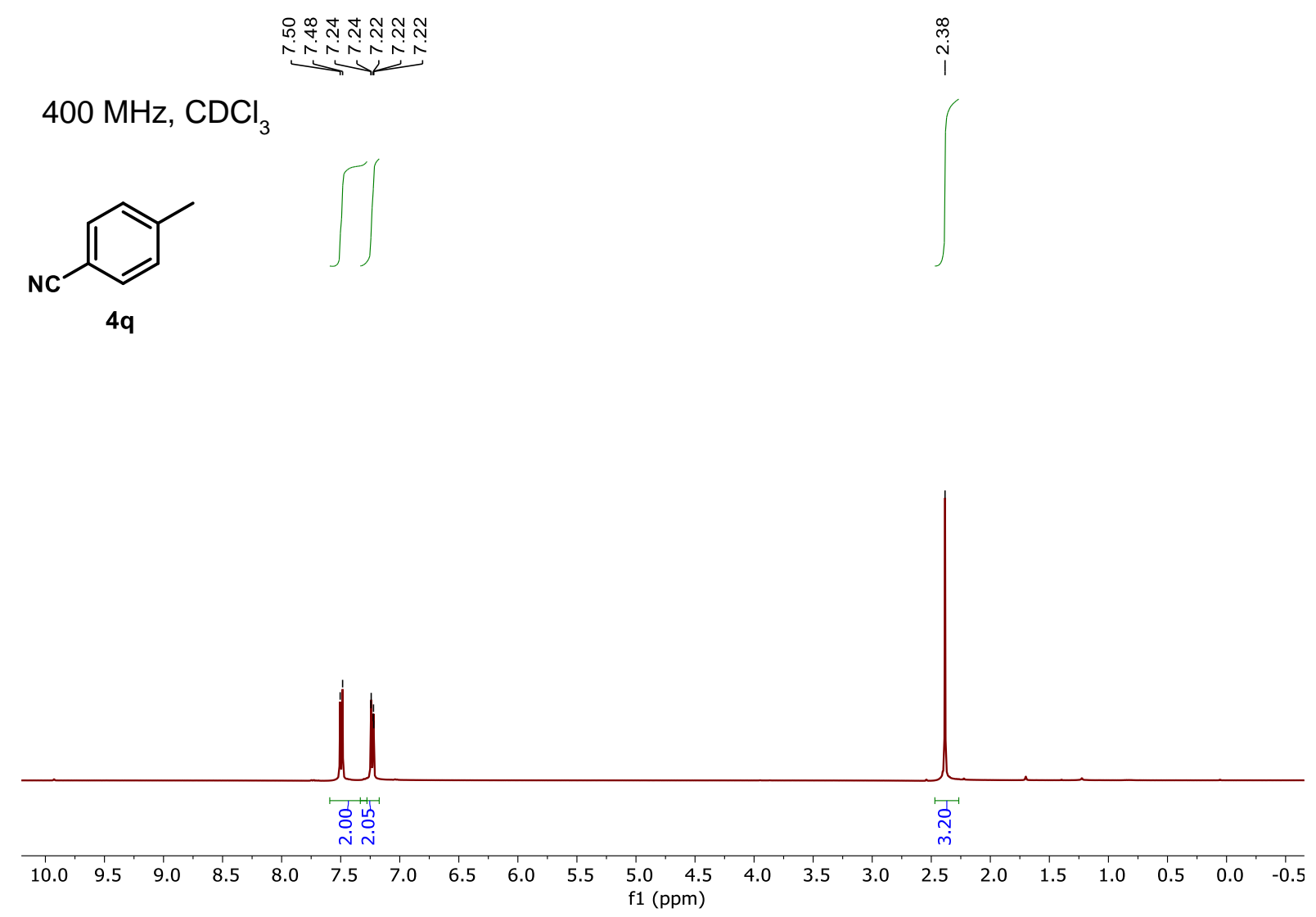

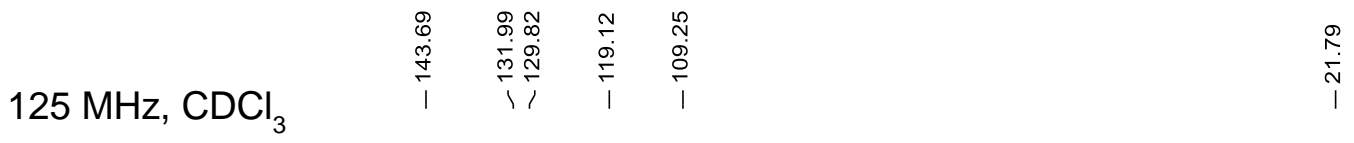<smiles>Cc1ccc(C#N)cc1</smiles>

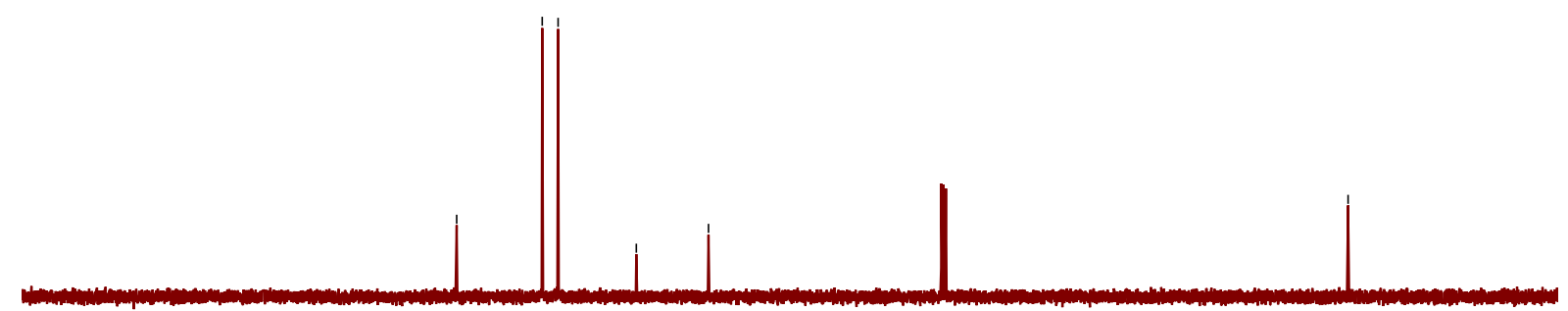

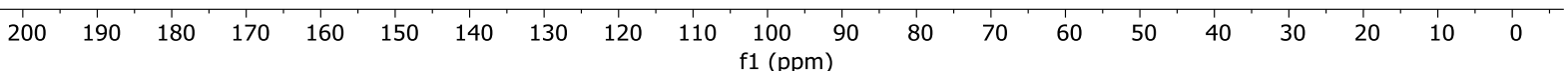


$500 \mathrm{MHz}^{\mathrm{CDCl}_{3}}$<smiles>COc1cc(CCc2cc(OC)c(OC)c(OC)c2)cc(OC)c1OC</smiles>

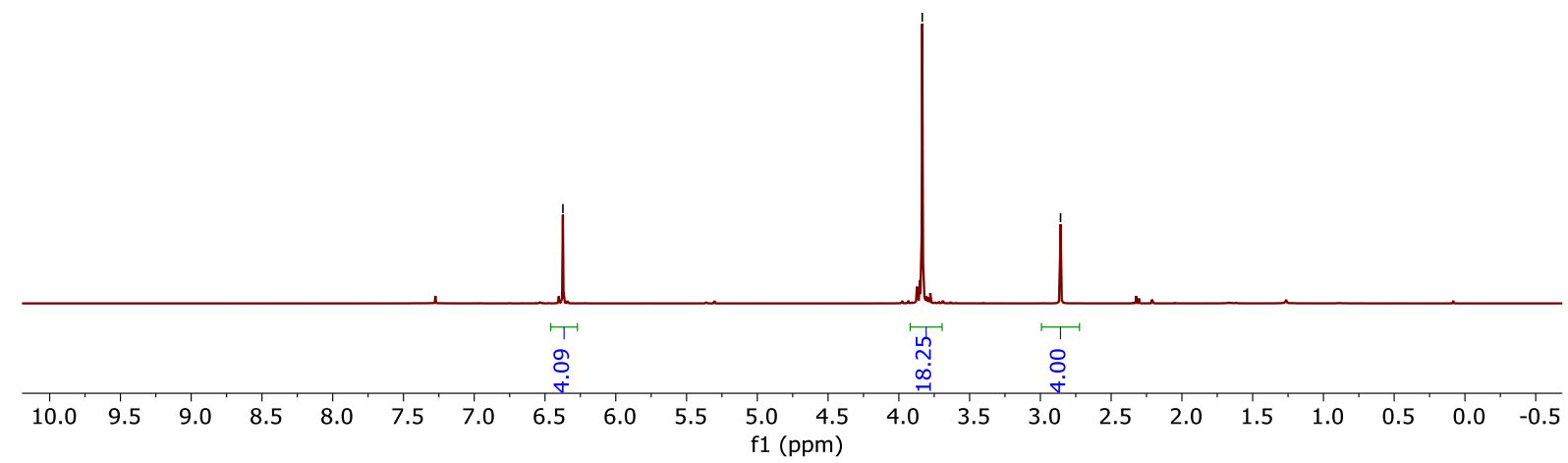

$125 \mathrm{MHz} \mathrm{CDCl}_{3}$

\begin{tabular}{|c|c|c|}
\hline 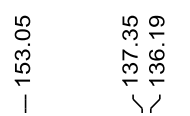 & 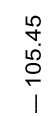 & $\begin{array}{ll}1 & 0 \\
0 & 0 \\
0 & 0 \\
0 & 0 \\
1 & 1\end{array}$ \\
\hline
\end{tabular}<smiles>COc1cc(CCc2cc(OC)c(OC)c(OC)c2)cc(OC)c1OC</smiles>

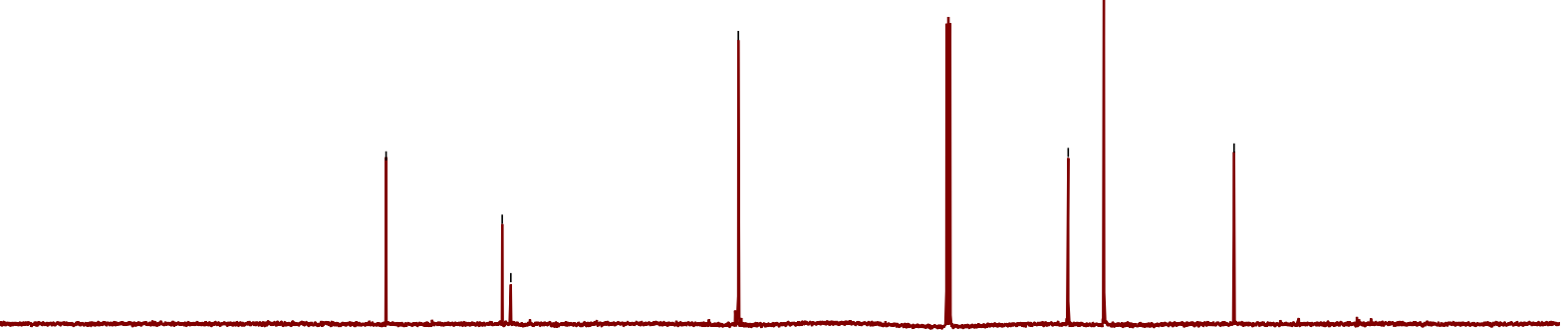

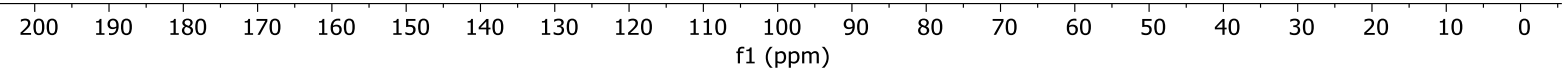




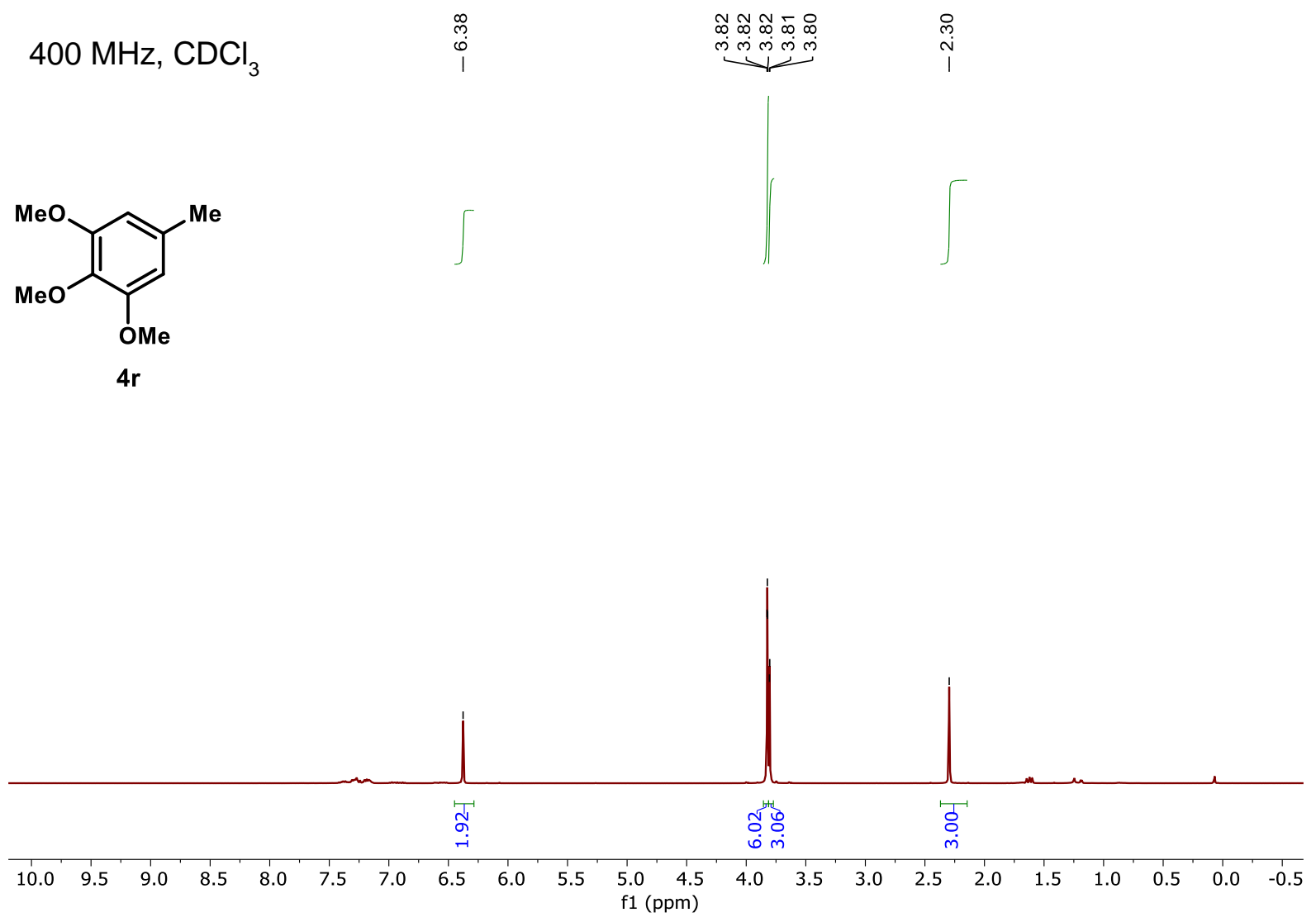




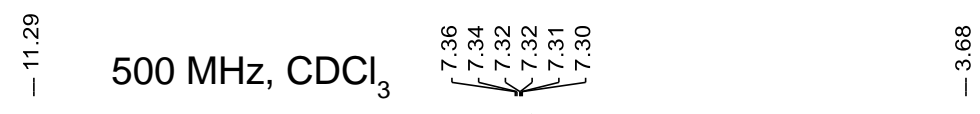<smiles>O=C(O)Cc1ccccc1</smiles>

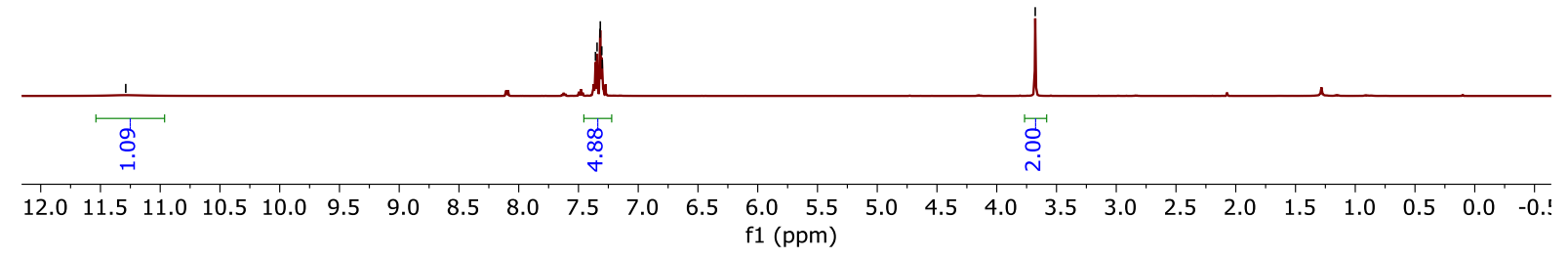

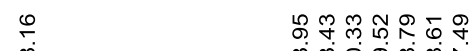

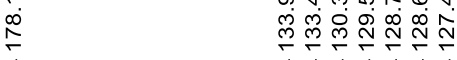

$125 \mathrm{MHz} \mathrm{CDCl}_{3}$

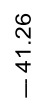<smiles>O=C(O)Cc1ccccc1</smiles>

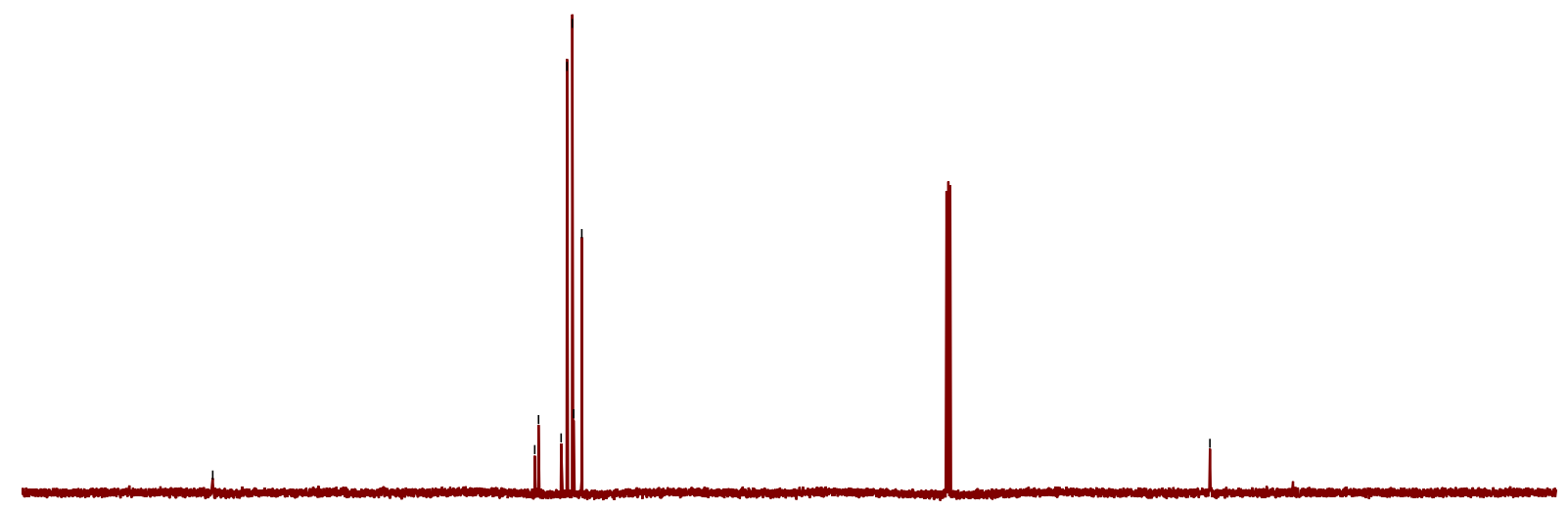

$\begin{array}{llllllllllllllllllllllll}200 & 190 & 180 & 170 & 160 & 150 & 140 & 130 & 120 & 110 & 100 & 90 & 80 & 70 & 60 & 50 & 40 & 30 & 20 & 10 & 0\end{array}$ 

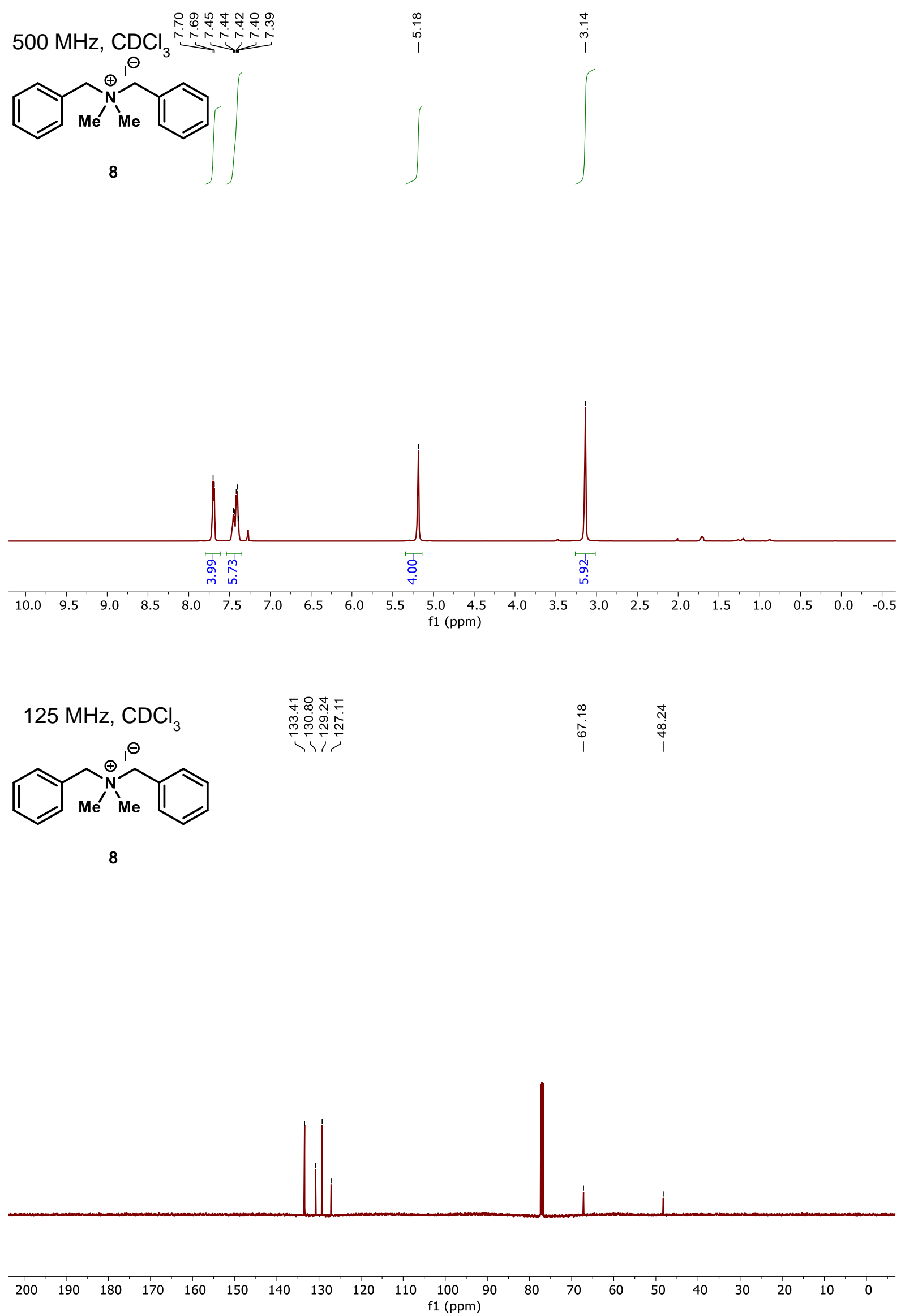

$\mathrm{S} 50$ 


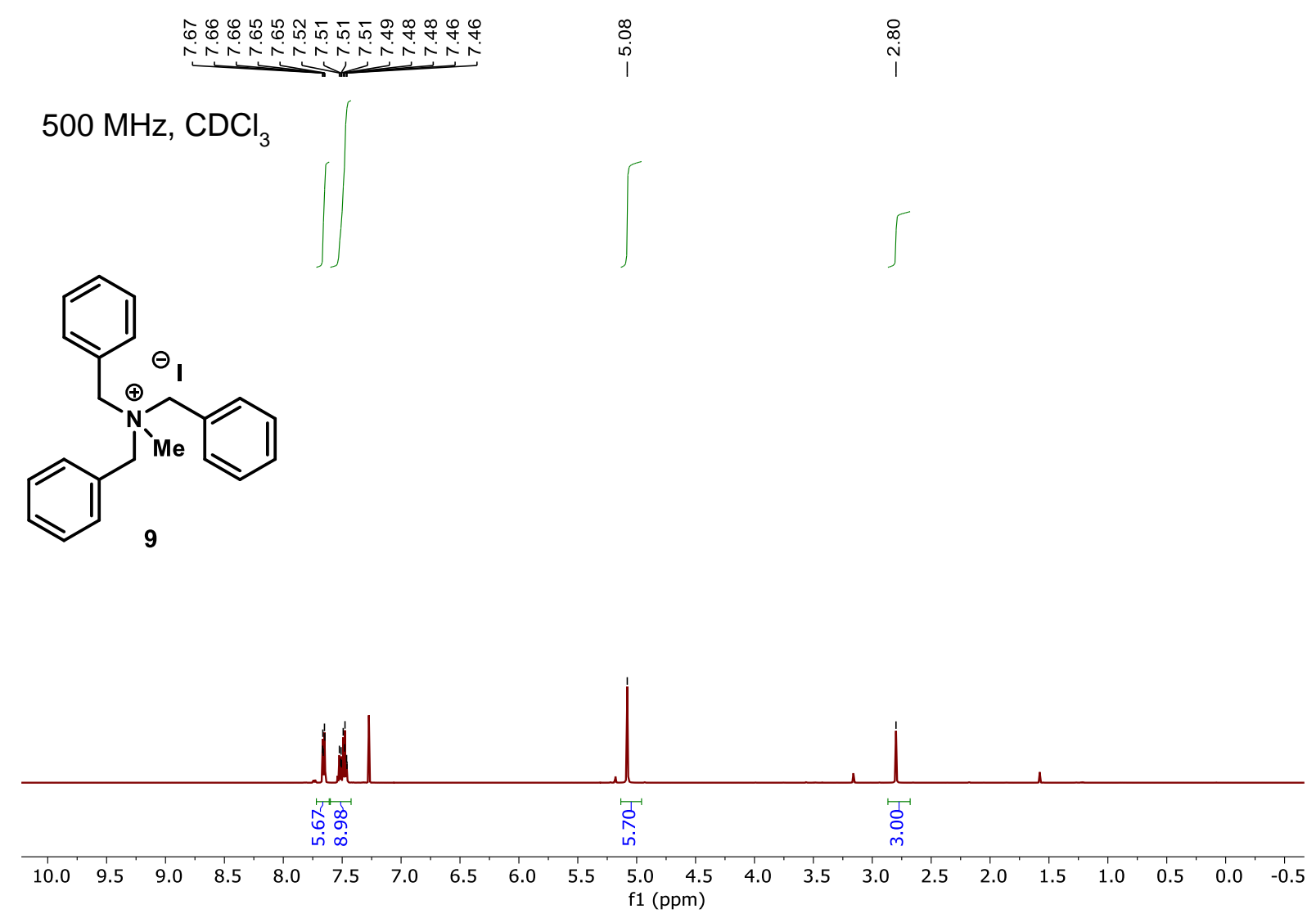

$500 \mathrm{MHz}, \mathrm{CDCl}_{3}$
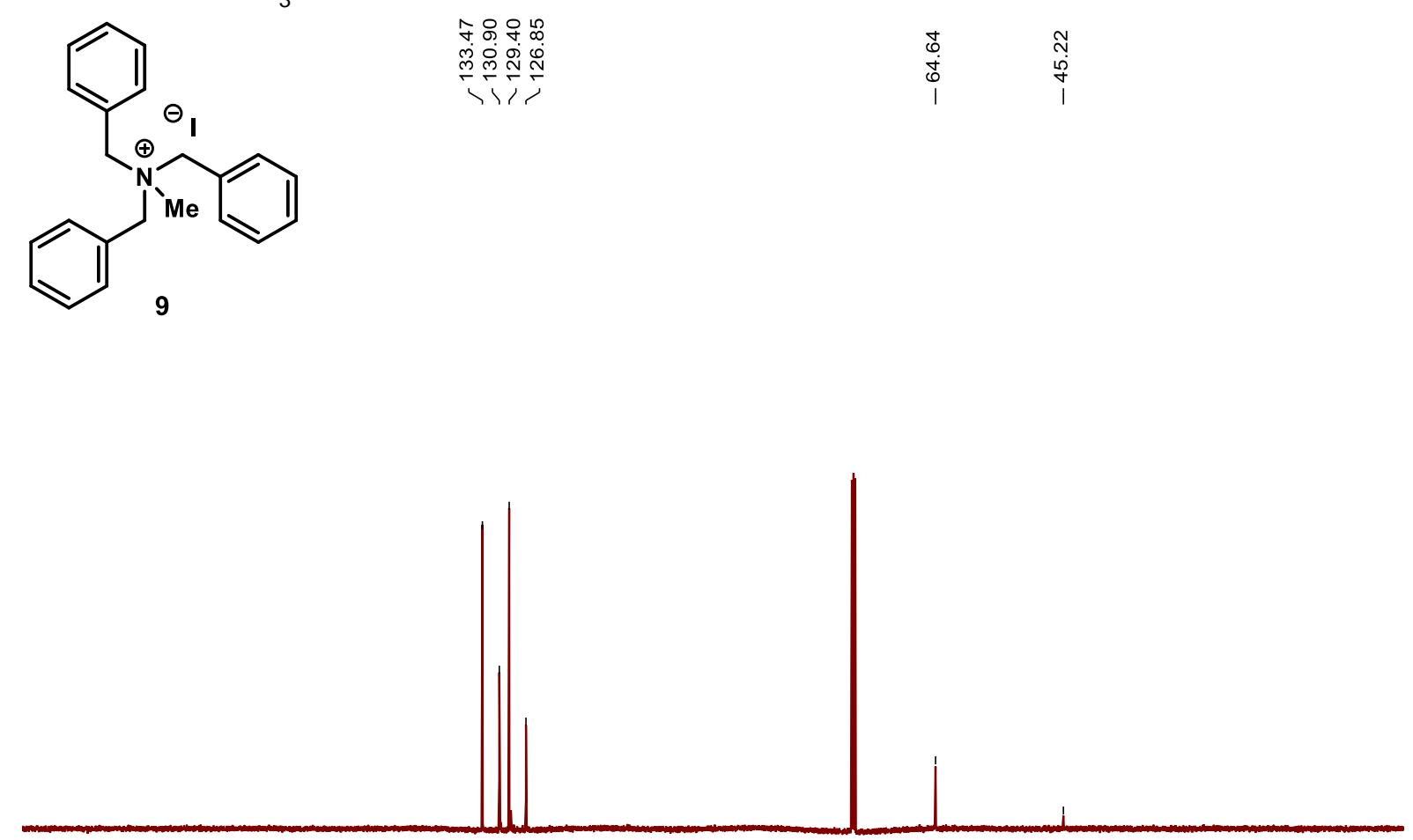

$\begin{array}{lllllllllllllllllllll}200 & 190 & 180 & 170 & 160 & 150 & 140 & 130 & 120 & 110 & \begin{array}{c}100 \\ \mathrm{f} 1(\mathrm{ppm})\end{array} & 90 & 80 & 70 & 60 & 50 & 40 & 30 & 20 & 10 & 0 \\ \mathrm{~S} 51\end{array}$ 

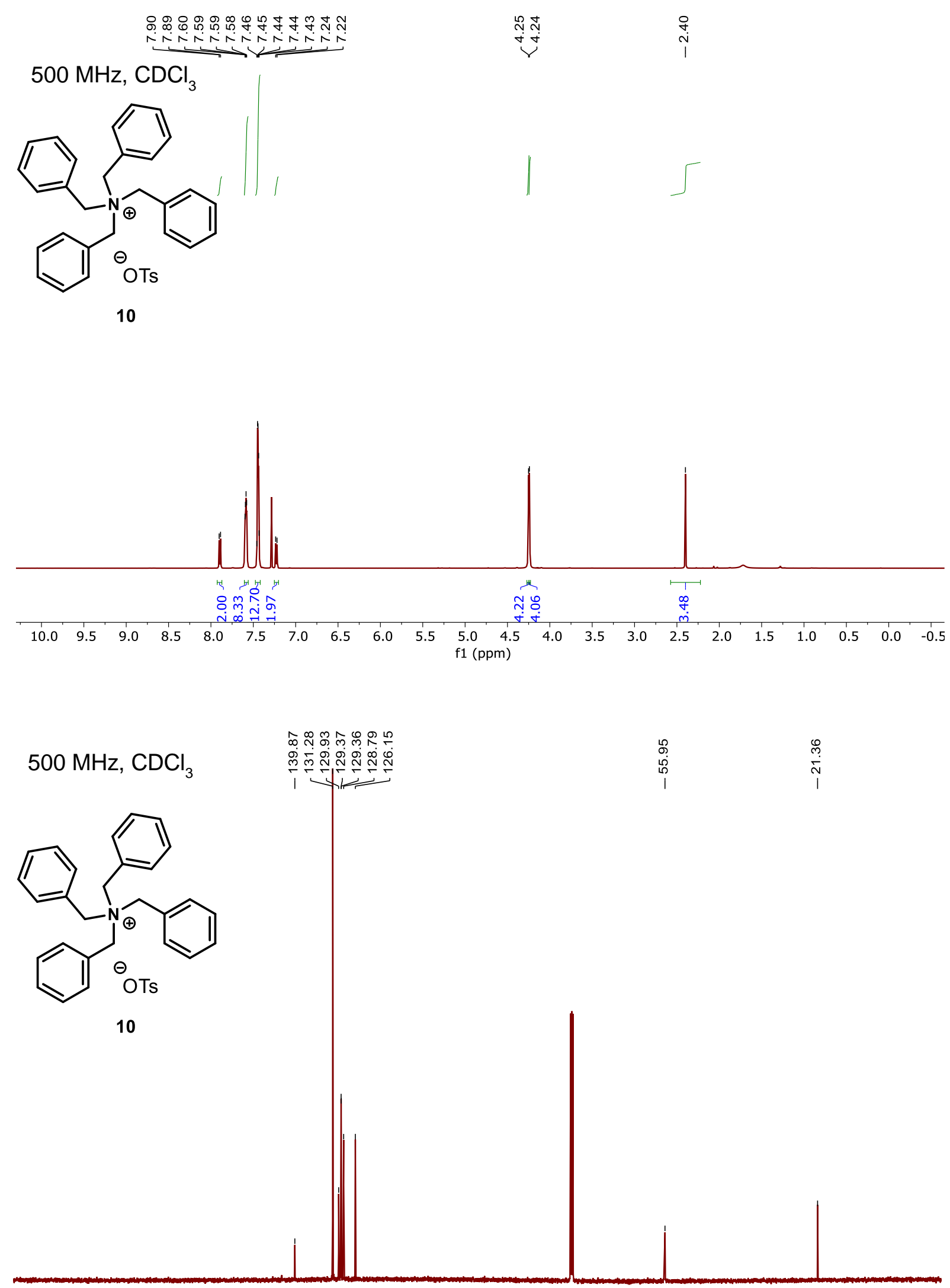

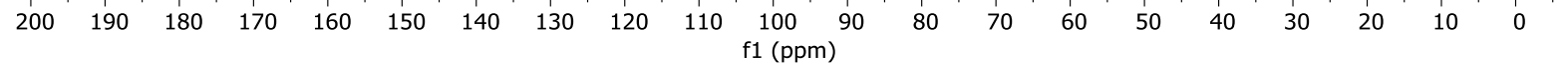




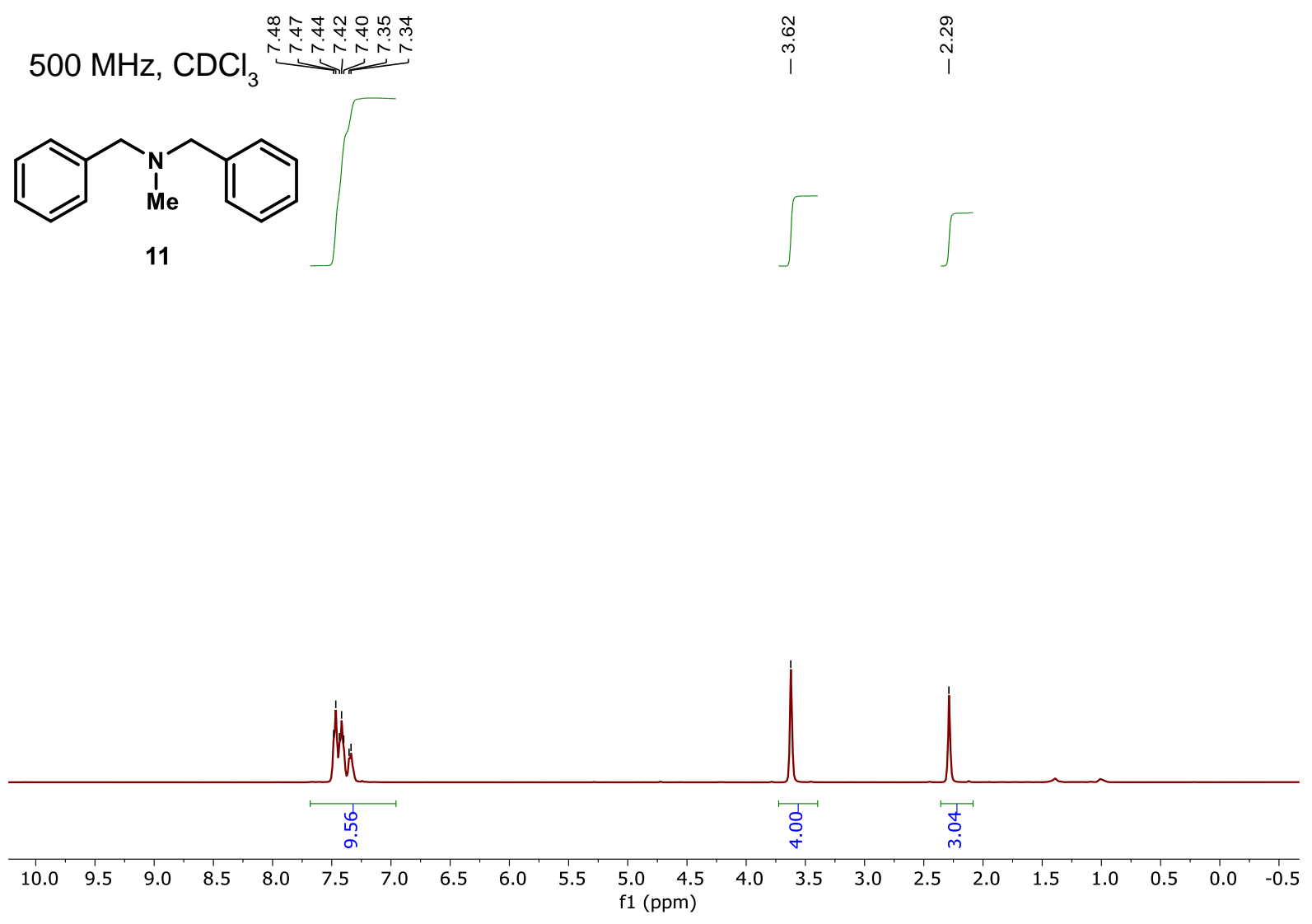

$125 \mathrm{MHz}, \mathrm{CDCl}_{3} \quad$ 施<smiles>CN(Cc1ccccc1)Cc1ccccc1</smiles>

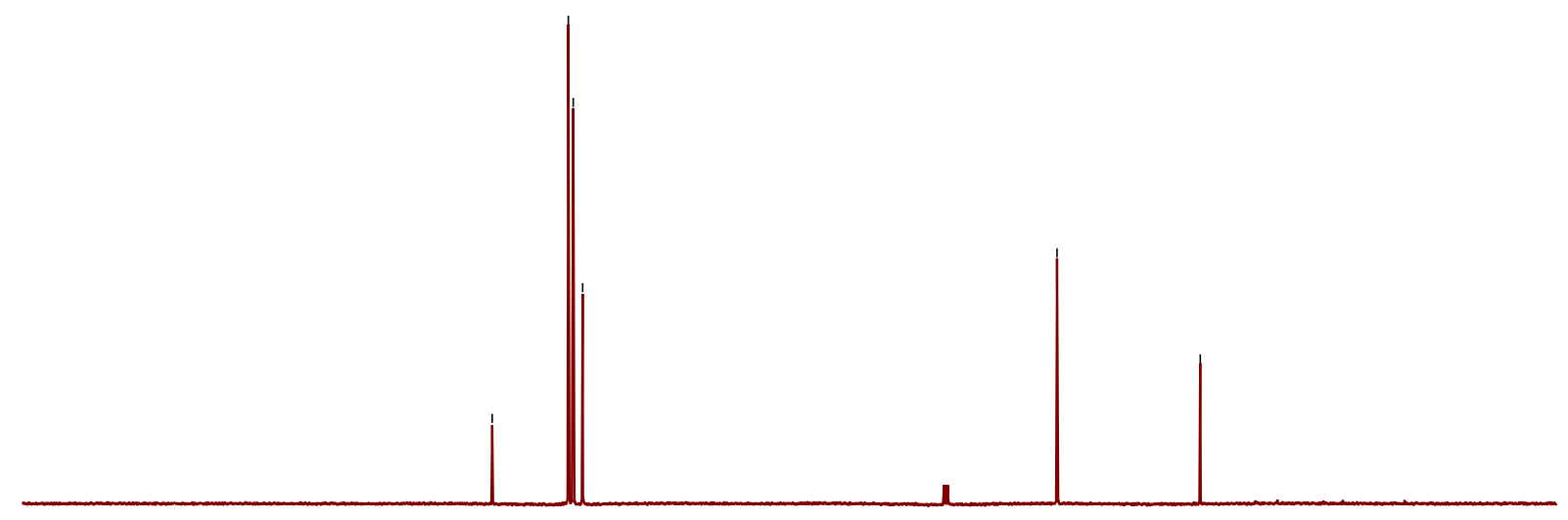

$\begin{array}{llllllllllllllllllllll}200 & 190 & 180 & 170 & 160 & 150 & 140 & 130 & 120 & 110 & \begin{array}{c}100 \\ \mathrm{f} 1(\mathrm{ppm})\end{array} & 90 & 80 & 70 & 60 & 50 & 40 & 30 & 20 & 10 & 0\end{array}$ 


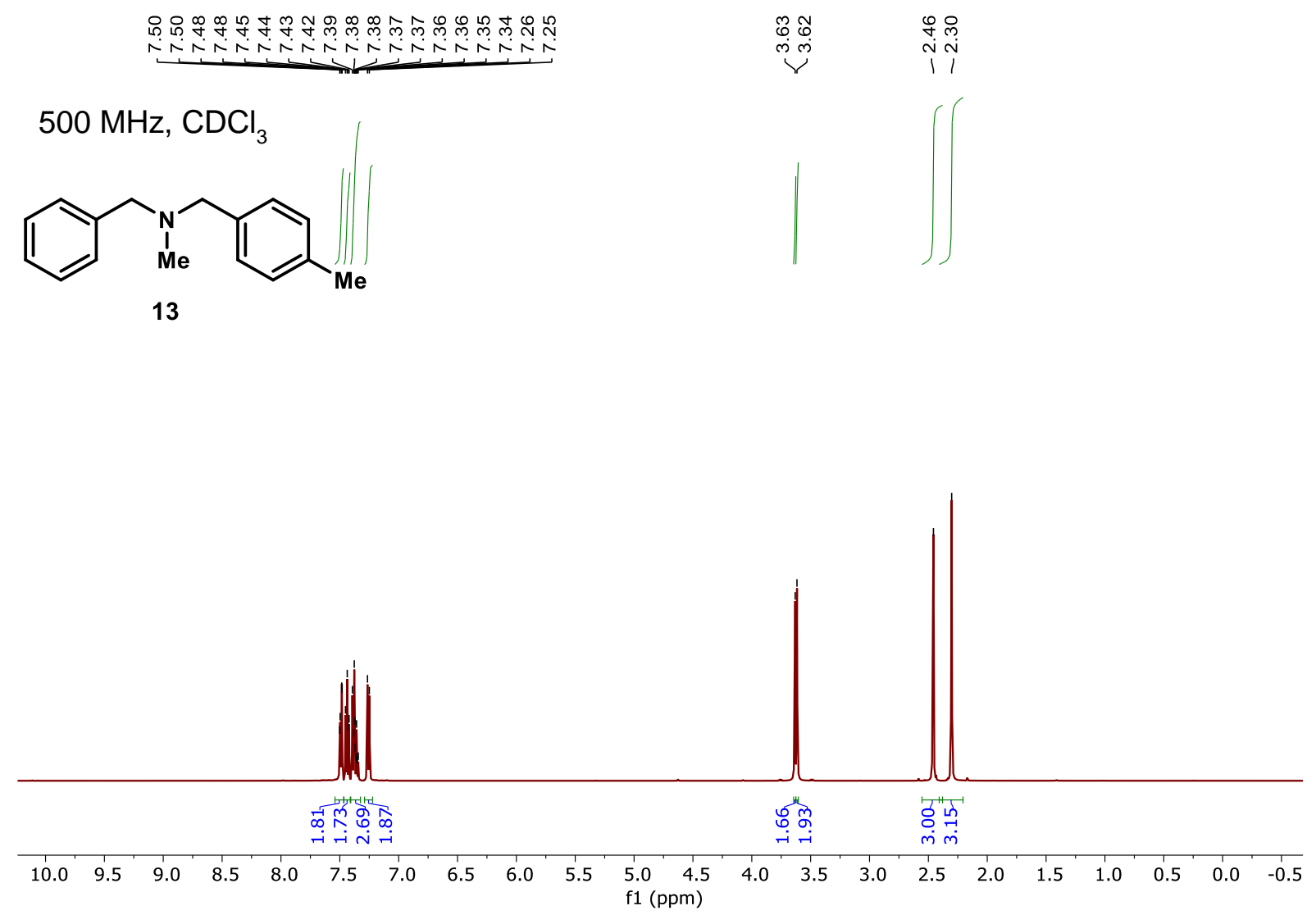

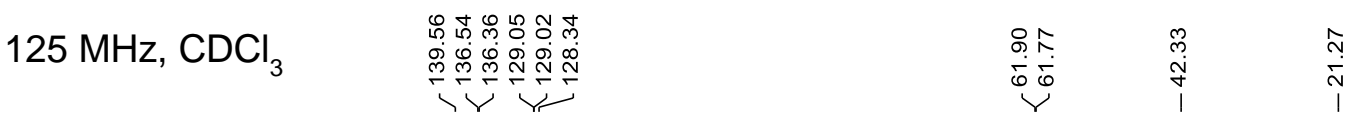<smiles>Cc1ccc(CN(Cc2ccccc2)S(C)(=O)=O)cc1</smiles>

13

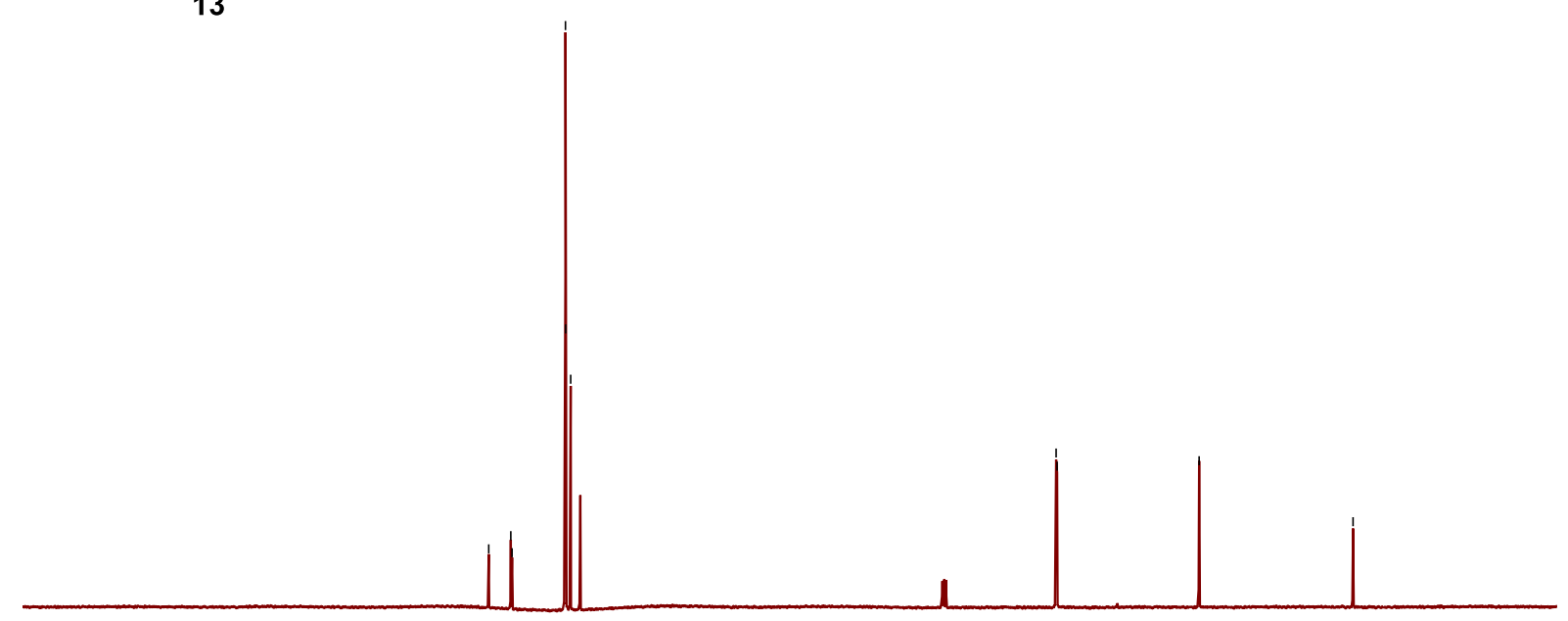

$\begin{array}{lllllllllllllllllllll}200 & 190 & 180 & 170 & 160 & 150 & 140 & 130 & 120 & 110 & 100 & 90 & 80 & 70 & 60 & 50 & 40 & 30 & 20 & 10 & 0\end{array}$ 
$500 \mathrm{MHz}, \mathrm{CDCl}_{3}$<smiles>C#CCCOCc1ccccc1Cc1ccc(C)cc1</smiles>

14

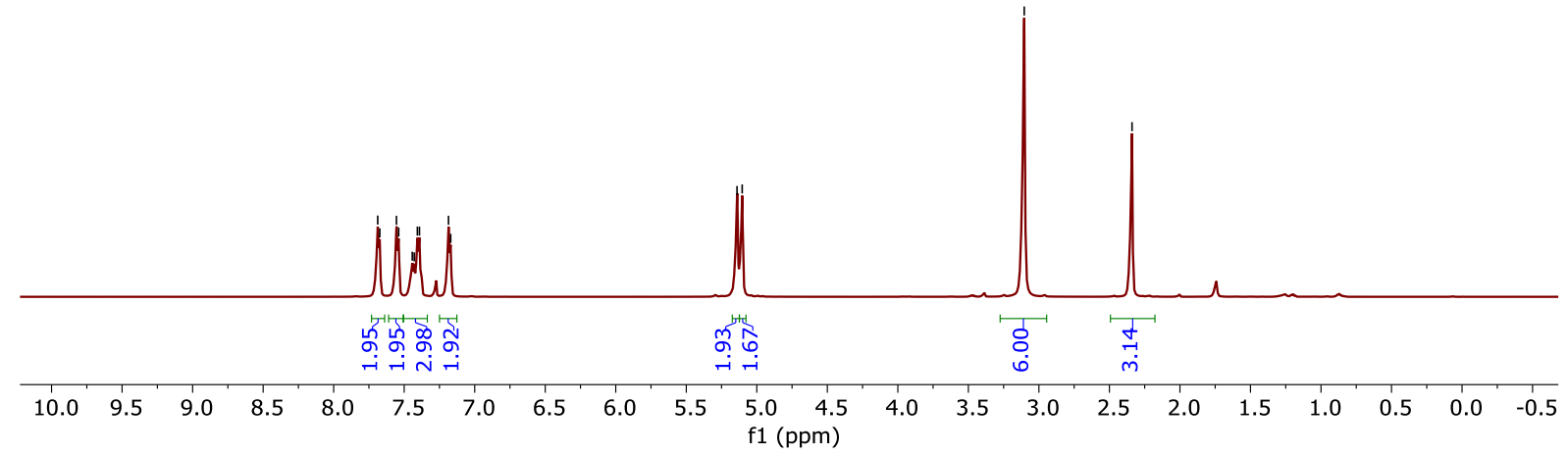<smiles>Cc1ccc(C[N+](C)([O-])Cc2ccccc2)cc1</smiles>

14

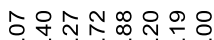

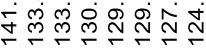

$125 \mathrm{MHz}, \mathrm{CDCl}_{3}$

? 


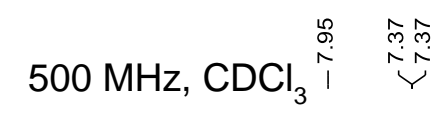

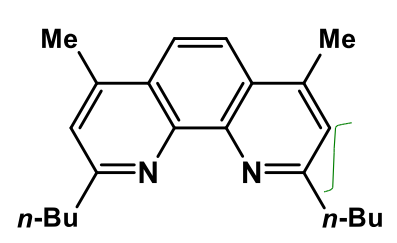

L5

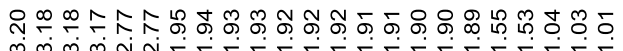

药

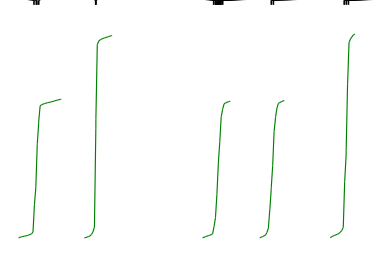

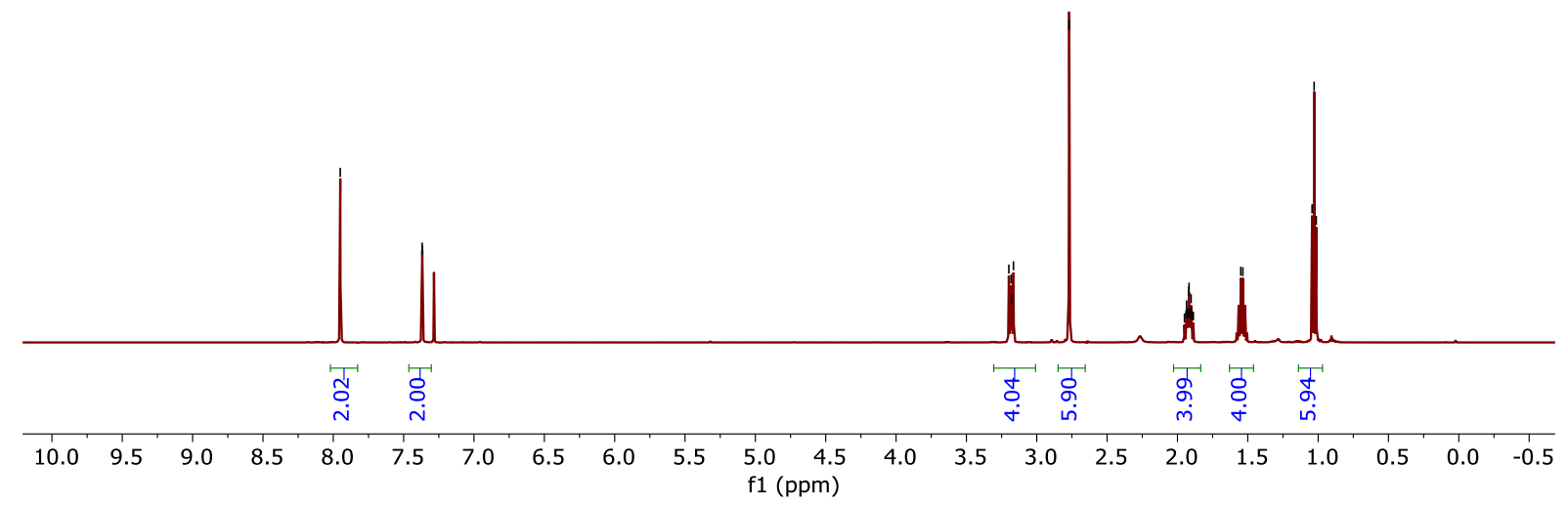




\section{VI. ${ }^{1} \mathrm{H}$ NMR and GC/MS trace for entry A in Fig. S5}
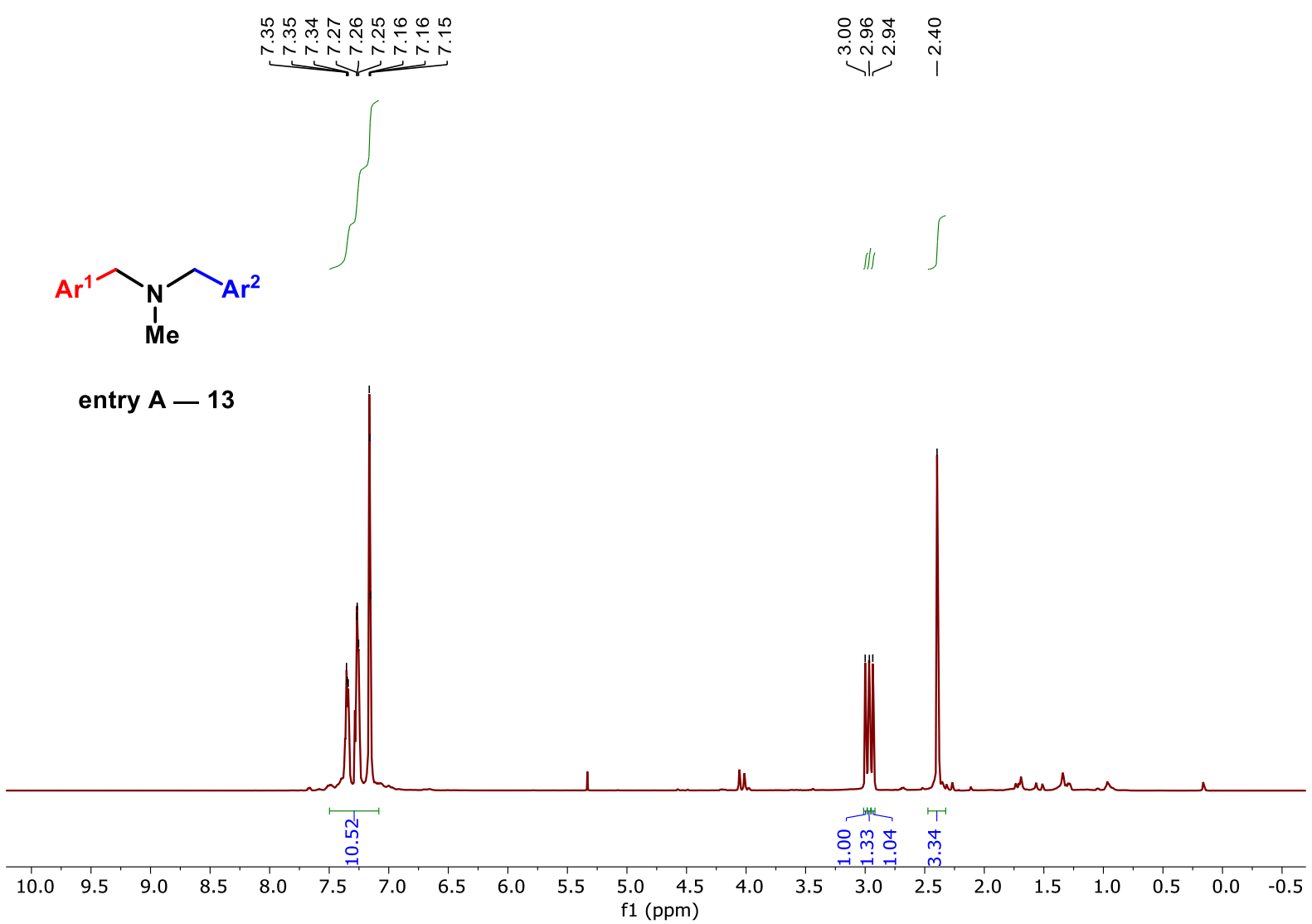

GC-MS method details: GC analyses were performed on an Agilent 5975C VL MSD GC equipped with dual DB-5MS columns (30 $\mathrm{m} \times 250 \mu \mathrm{m} \times 25 \mu \mathrm{m})$, triple FID detectors, and with helium carrier gas. The analysis method used in all cases was $1 \mu \mathrm{L}$ inj. of sample, inj. temp of $250^{\circ} \mathrm{C}, 25: 1$ split ratio, initial inlet pressure was 7.65 psi but varied as the column flow was held constant at 1.0 $\mathrm{mL} / \mathrm{min}$ for the duration of the run. Initial oven temperature of $50^{\circ} \mathrm{C}$ was held for 3 min followed by a temperature ramp up to $280{ }^{\circ} \mathrm{C}$ at $40 \stackrel{\circ}{\circ} \mathrm{C} / \mathrm{min}$ and finally the temperature was held at $300^{\circ} \mathrm{C}$ for $8 \mathrm{~min}$. Total run time was $\sim 24 \mathrm{~min}$. FID temperature was $300^{\circ} \mathrm{C}$.

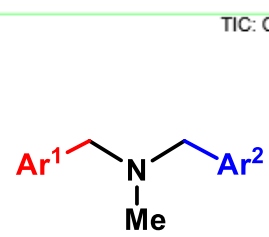

C: CIN-02-230D.Dldata.ms

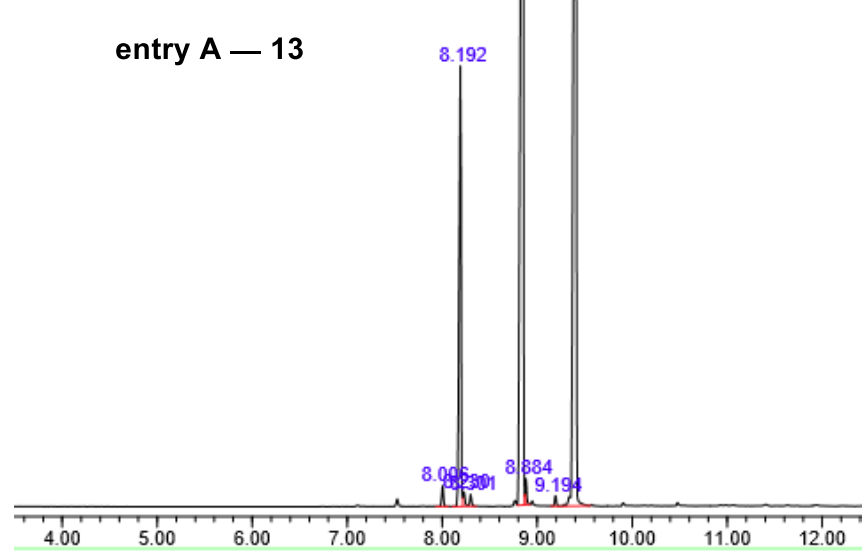

peak 1 - elution, 8.19 min, 2a: calcd. for $\mathrm{C}_{14} \mathrm{H}_{14}\left[\mathrm{M}^{+}\right]$: 182.1 , found: 182.2 peak 2 - elution, $8.85 \mathrm{~min}, 16$ : calcd. for $\mathrm{C}_{15} \mathrm{H}_{16}[\mathrm{M}+]$ : 196.1 , found: 196.2 peak 3 - elution, $9.41 \mathrm{~min}, \mathbf{2 b}$ : calcd. for $\mathrm{C}_{16} \mathrm{H}_{18}\left[\mathrm{M}^{+}\right]$: 210.1 ; found: 210.2 